\title{
Design Guidelines for Spatial Modulation
}

\author{
Ping Yang, Marco Di Renzo, Senior Member, IEEE, Yue Xiao, Shaoqian Li, Senior Member, IEEE, and
}

Lajos Hanzo, Fellow, IEEE

\begin{abstract}
4 Abstract-A new class of low-complexity, yet energy-efficient 5 Multiple-Input Multiple-Output (MIMO) transmission tech6 niques, namely, the family of Spatial Modulation (SM) aided 7 MIMOs (SM-MIMO), has emerged. These systems are capable of 8 exploiting the spatial dimensions (i.e., the antenna indices) as an 9 additional dimension invoked for transmitting information, apart 10 from the traditional Amplitude and Phase Modulation (APM). SM 11 is capable of efficiently operating in diverse MIMO configurations 12 in the context of future communication systems. It constitutes a 13 promising transmission candidate for large-scale MIMO design 14 and for the indoor optical wireless communication while relying 15 on a single-Radio Frequency (RF) chain. Moreover, SM may be 16 also viewed as an entirely new hybrid modulation scheme, which is 17 still in its infancy. This paper aims for providing a general survey 18 of the SM design framework as well as of its intrinsic limits. In 19 particular, we focus our attention on the associated transceiver 20 design, on spatial constellation optimization, on link adaptation 21 techniques, on distributed/cooperative protocol design issues, and 22 on their meritorious variants.
\end{abstract}

23 Index Terms-Cooperative communications, large-scale 24 MIMO, link adaptation, space-time coding, spatial modulation.

\section{INTRODUCTION}

26 ULTIPLE-INPUT multiple-output (MIMO) systems are 27 capable of achieving a capacity gain and/or diversity 28 gain, which is based on striking a beneficial trade-off, de29 pending on the near-instantaneous channel conditions [1]-[4]. 30 Hence they have been adopted in most of the recent com31 munication standards, such as IEEE 802.11n, IEEE 802.16e, 32 and 3GPP Long-Term Evolution (LTE) [5], [6]. In a wireless 33 MIMO transmission system, the transmission technique em34 ployed plays an important role in determining the achievable 35 system performance. Recently, the conventional spatial-domain 36 MIMO transmission techniques have been extended to the time37 domain, the frequency-domain as well as to their combinations

Manuscript received October 15, 2013; revised March 19, 2014; accepted May 23, 2014. This work was supported by the National Science Foundation of China under Grant 61101101, by the European Research Council's Advanced Fellow Grant, by the National Basic Research Program of China under Grant 2013CB329001, and by the European Commission under the auspices of the FP7-PEOPLE ITN-GREENET Project under Grant 264759.

P. Yang, Y. Xiao, and S. Li are with the National Key Laboratory of Science and Technology on Communications, University of Electronic Science and Technology of China, Chengdu 611731, China (e-mail: yplxw@163.com; 1sq@uestc.edu.cn; xiaoyue@uestc.edu.cn).

M. Di Renzo is with the Laboratory of Signals and Systems (L2S), French National Center for Scientific Research (CNRS), University of Paris-Sud XI, 91192 Gif-sur-Yvette, France (e-mail: marco.direnzo@1ss.supelec.fr).

L. Hanzo is with the School of Electronics and Computer Science, University of Southampton, Southampton SO17 1BJ, U.K. (e-mail: 1h@ecs.soton.ac.uk).

Color versions of one or more of the figures in this paper are available online at http://ieeexplore.ieee.org.

Digital Object Identifier 10.1109/COMST.2014.2327066
[7], [8]. In order to efficiently exploit the associated grade of 38 freedom offered by MIMO channels, a meritorious transmis- 39 sion technique should be designed to satisfy a diverse range 40 of practical requirements and to strike an attractive tradeoff 41 amongst the conflicting factors of the computational complexity 42 imposed, the attainable bit error ratio (BER) and the achievable 43 transmission rate [9], [10].

In the diverse family of MIMO techniques, the recently pro- 45 posed spatial modulation (SM) [11] (which was referred to as 46 Information-Guided Channel Hopping (IGCH) modulation in 47 [12]) is particularly promising, since it is capable of exploiting 48 the indices of the transmit antennas (TAs) as an additional 49 dimension invoked for transmitting information, apart from 50 the traditional Amplitude and Phase Modulation (APM) [13]. 51 At a given Signal to Noise Ratio (SNR), the throughput of 52 the SM-MIMO may potentially become higher than that of 53 Space-Time Coding (STC) [14], but this is not necessarily its 54 most prominent benefit, because in SM only a single TA is 55 activated at any time instant. Hence SM is capable of dispensing 56 with the requirement of multiple Radio Frequency (RF) chains, 57 therefore relaxing the Inter-Antenna-Synchronization (IAS) 58 specifications, whilst mitigating the Inter Antenna Interference 59 (IAI) of conventional MIMO techniques [15]. Additionally, 60 the single-RF design is capable of reducing the total power 61 consumption. In fact, only a single power amplifier is needed 62 for implementing SM-MIMO systems, which is typically re- 63 sponsible for the vast majority of power dissipation at the 64 transmitter [16], [17]. Another advantage of SM is that it may 65 be flexibly configured for diverse transmit and receive antenna 66 constellations, especially for the challenging scenario of asym- 67 metric/unbalanced MIMO systems, whose channel matrix is 68 rank-deficient [15].

Due to the above-mentioned advantages, SM constitutes an 70 attractive option for the emerging family of large-scale MIMO 71 systems [18], [19]. As a further advance, the principle of 72 SMs was also extended to indoor optical wireless communi- 73 cation in [20]-[23], which relies on optical transmissions for 74 conveying information. Altogether, SM constitutes a promis- 75 ing low-complexity energy-efficient MIMO transmission tech- 76 nique, which relies on a low-cost transceiver and is capable 77 of efficiently operating in diverse MIMO configurations in 78 the context of future communication systems. Recently, the 79 potential benefits of SM have been validated not only via 80 simulations [11], [14] but also by experiments [24]-[26]. The 81 benefits of SM-MIMOs aided wireless communications are 82 summarized in Fig. 1. In the sequel, they are characterized in 83 more detail.

The wide-ranging simulation based and analytical studies 85 disseminated in [27]-[34] have characterized some of the 86 


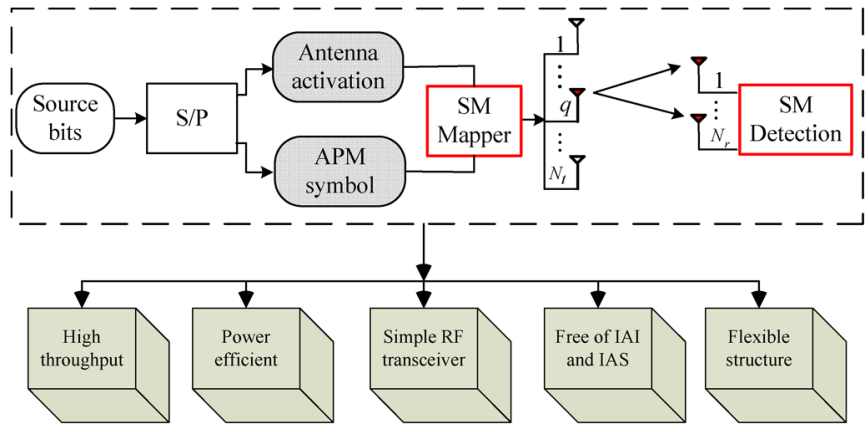

Fig. 1. Benefits of SM-MIMOs for wireless communications.

87 fundamental properties of SM related to the channel's corre88 lation [27], [28]. Furthermore, the issues of achieving transmit 89 diversity [29], the effects of power imbalance [30], the specific 90 choice of the APM scheme used [31], the impact of the specific 91 channel encountered [29], [32] as well as the effects of channel 92 estimation errors [33], [34] were also characterized. It was 93 found that the performance of SM-MIMOs is highly dependent 94 on the specific type of the APM scheme used. For example, as 95 a hybrid modulation scheme, which combines the classic APM 96 constellation and the spatial-domain (SD) constellation, the 97 SM's achievable performance depends both on the minimum 98 Euclidean distance (ED) of the APM constellation employed, 99 as well as the on absolute values of the modulated symbols 100 [29]. Hence, a suitable APM scheme has to be carefully de101 signed for exploiting the benefits of this hybrid modulation 102 scheme.

103 On the other hand, it was also noted that the conventional 104 open-loop SM schemes [11], [12] only offer receive-diversity 105 gains. Hence there is also a paucity of SM-MIMO solutions on 106 how to increase the system's robustness to time-varying channel 107 conditions with the aid of either open or closed-loop transmit108 symbol design techniques [14]. Additionally, unlike in conven109 tional MIMO techniques, the transmit vectors of SM-MIMO 110 schemes are sparsely populated, since they have mostly zero 111 values [11]. This constraint makes SM rather different from 112 classic Space Time Block Codes (STBC) [35] designed for 113 achieving a diversity gain or from Spatial Division Multiplexing 114 (SDM) [36] conceived for attaining a multiplexing gain as well 115 as from the hybrid SDM-STBC schemes [37] aiming for strik116 ing a compromise. In order to increase the robustness of SM117 MIMO systems, the classic time-variant parameter adaptation 118 techniques [38], such as power allocation and precoding [39]119 [41], which were proposed for conventional MIMO techniques 120 may not be directly applied to SM schemes owing to their 121 specific transmission mode.

122 In this treatise, we provide a general survey of the SM design 123 framework as well as of its intrinsic limitations. We summarize 124 the most recent research achievements and outline their poten125 tial applications, as well as their impediments, which have to be 126 overcome before these MIMO technique may be used as main127 stream solutions in practical systems. In particular, we focus 128 our attention on the associated transceiver design, on spatial 129 constellation optimization, on link adaptation techniques, on 130 distributed/cooperative protocol design and on their meritorious 131 variants.
The paper is organized as follows. Section II reviews the con- 132 ventional SM technique and its relevant variants, emphasizing 133 the flexible transceiver design techniques conceived for striking 134 an attractive trade-off amongst the often conflicting system 135 requirements. The spatial constellation optimization and the as- 136 sociated link adaptation techniques are presented in Sections III 137 and IV, respectively. Section V surveys the family of relay 138 aided SM schemes, which exploits the particular information 139 transmission characteristics of SM and introduces the class of 140 SM-related systems designed for dispersive channels. Finally, 141 Section VI concludes the paper.

142

Although the list of the references is not exhaustive, the 143 papers cited as well as the references therein can serve as 144 a good starting point for further reading. In particular, there 145 are several tutorial-style articles, [8], [14] and [15], which 146 tend to have quite a different focus. To be specific, in [8], 147 the authors have reviewed diverse MIMO arrangements and 148 then focus on a new class of MIMOs based on the concept 149 of space-time shift keying. In [14], the authors have evaluated 150 the advantages and disadvantages of SM with respect to other 151 popular MIMO schemes and summarized some early research 152 achievements. Moreover, in [15], some of the co-authors of 153 this treatise have provided a comprehensive survey of spa- 154 tial modulation research, with an emphasis on a generalized 155 transceiver scheme combining spatial modulation with spatial 156 multiplexing and space-time block coding in order to increase 157 either the spectral efficiency or the diversity gain. The price to 158 pay for this flexibility is the need for multiple radio frequency 159 chains. Moreover, in [15] the authors emphasized the energy 160 efficiency of MIMO-based transmission schemes and the first 161 SM-MIMO-based testbed results recorded both in realistic 162 outdoor and indoor propagation environments were reported. 163 Suffice to say that [15] was conceived for stimulating cross- 164 disciplinary research across different communities, whilst this 165 contribution is targeted at readers with a background in wireless 166 communications, who might like to delve into SM-research. 167

Against this background, this contribution firstly provides a 168 succinct description of the basic spatial modulation principle. 169 To be specific, the SM techniques are classified and then the 170 corresponding detection techniques are categorized with the aid 171 of tables for explicit clarity. Moreover, this paper is more fo- 172 cused on illustrating those results that lead to new design guide- 173 lines, as exemplified by the constellation optimization issues 174 of SM. Furthermore, there is a special emphasis on powerful 175 adaptive modulation aided SM and on precoding aided SM. 176 A range of performance metrics are introduced for optimizing 177 spatial modulation, which rely either on the available long-term 178 statistical or on the near-instantaneous knowledge about the 179 channel.

\section{TRAnsceiver Design of SM-MIMO}

\section{A. The Transmitter Design of SM}

In this section, we consider the $\left(N_{t} \times N_{r}\right)$-element SM- 183 MIMO system, which relies on $N_{t}$ transmit and $N_{r}$ receive 184 antennas, while communicating over frequency-flat Rayleigh 185 fading channels. The conventional bit-to-symbol mapping rule 186 


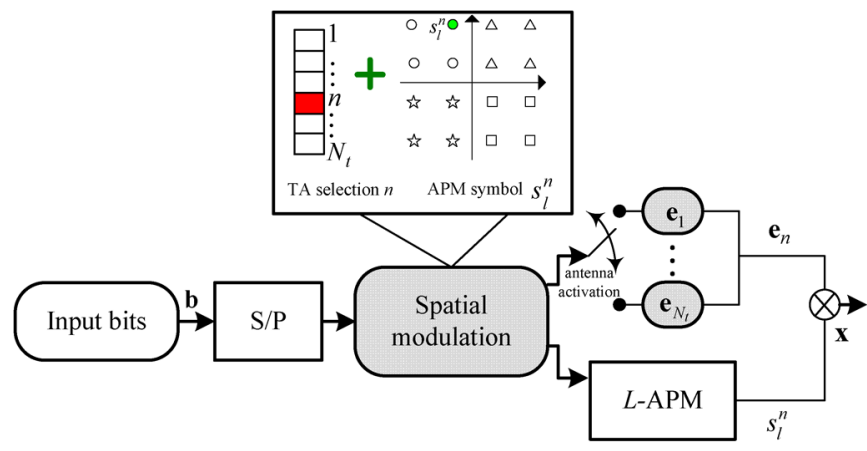

Fig. 2. SM bit-to-symbol mapping rule.

187 [11] of SM is portrayed in Fig. 2, which can be divided into 188 three steps as follows:

189 Algorithm 1: Bit-to-symbol mapping principle of the SM 190 transmitter of Fig. 2

191 1) First, the information bit stream is divided into vectors containing $m_{\text {all }}=\log _{2}\left(L \cdot N_{t}\right)$ bits each.

2) Next, each vector is further split into two sub-vectors of $\log _{2}\left(N_{t}\right)$ and $\log _{2}(L)$ bits each. The bits in the first subvector are used for activating a unique TA for transmission, while the bits in the second sub-vector are mapped to an APM symbol $s_{l}^{n}$. Note that the TA activation process can be described by the $N_{t}$-dimensional standard basis vector $\mathbf{e}_{n}\left(1 \leq n \leq N_{t}\right)$ (i.e., $\mathbf{e}_{1}=[1,0, \cdots, 0]^{T}$ ).

3) Finally, the transmitted symbol $x$ is comprised of the APM symbol $s_{l}^{n}$ emitted from the activated TA $n$. The resultant modulated symbol can be formulated as $\mathbf{x}=$ $s_{l}^{q} \mathbf{e}_{q} \in \mathbb{C}^{N_{t} \times 1}$.

204 The corresponding vector-based signal received at the SM205 MIMO receiver is given by

$$
\mathbf{y}=\mathbf{H x}+\mathbf{n}=\mathbf{h}_{n} s_{l}^{n}+\mathbf{n}
$$

206 where $\mathbf{H}$ is an $\left(N_{r} \times N_{t}\right)$-element channel matrix, $\mathbf{h}_{n}$ is the 207 nth column of $\mathbf{H}$ and the elements of the $N_{r}$-dimensional 208 noise vector $\mathbf{n}$ are complex Gaussian random variables obeying $209 \mathcal{C N}\left(0, N_{0}\right)$.

\section{B. Variants of the SM Principle}

211 The first conference paper on SM was published in 2001 [45], 212 but its extensive research was mainly fueled by the pioneering 213 works of Haas et al. [42], Mesleh et al. [11], followed by 214 Sugiura et al. [43], Yang et al. [12] and Jeganathan et al. [44]. 215 Throughout its decade-long history, the SM concept has been 216 termed in different ways and it was extended to different scenar217 ios. A range of major contributions on the subject of SM and its 218 related variants are listed in Table I. Specifically, the concept of 219 SM was first touched upon in [45], where the distinct multipath 220 components were exploited for detection. In [42], a novel Or221 thogonal Spatial-Division Multiplexing (OSDM) scheme was proposed, which utilizes the index of the TAs as a means of 222 conveying additional source information. In [11], a beneficial 223 framework was established for the bit-to-symbol mapping rule 224 of SM. It was also demonstrated in [11] that SM may be 225 capable of attaining a better performance than other conven- 226 tional MIMO schemes, such as the Vertical Bell Laboratories 227 Layered Space-Time (V-BLAST) and STBC [4], even with- 228 out reducing the achievable data rate,. The above-mentioned 229 IGCH technique was proposed in [12] for achieving a high 230 throughput. Later, Space Shift Keying (SSK) [44] modulation 231 was conceived for relying exclusively on the TA indices to 232 convey information, whilst entirely dispensing with any classic 233 Phase Shift Keying (PSK)/Quadrature Amplitude Modulation 234 (QAM) signaling [13]. In a nutshell, all of the above-mentioned 235 schemes activate only a single TA at any instant in order to 236 maintain a low complexity, whilst mitigating to IAI and IAS 237 specifications, as well as reducing to total power consumed. 238

Motivated by the above concepts, various generalized ver- 239 sions of SM were proposed. First, as a natural extension of 240 SSK, the Generalized SSK (GSSK) scheme was proposed in 241 [46], which activates multiple TAs for the sake of achieving 242 an increased-rate data transmission. This extension has also 243 been incorporated into the SM scheme and two classes of 244 Generalized SM (GSM) schemes were obtained [47]-[49]. To 245 be specific, in [47] a class of GSM arrangements was proposed 246 for the sake of attaining increased transmit diversity gains, 247 which uses all the active TAs for transmitting the same APM- 248 modulated symbols. By contrast, in [48] and [49], another class 249 of GSM arrangements was proposed for attaining an increased 250 multiplexing gain, which uses the active transmit antennas to 251 carry different information symbols during each time slot. Note 252 that the above-mentioned generalized SM schemes of [46]- 253 [49] allow us to activate several-rather than only a single 254 antenna-at the transmitter for bit-to-symbol mapping, hence 255 they are capable of overcoming a specific constraint of SM, 256 namely that the number of TAs has to be a power of two. 257 Moreover, SM was combined with the classic STBC scheme 258 in [50] and with Trellis Coding (TC) in [51]-[53] in order to 259 take advantage of the benefits of both.

260

Recently, Space-Time Shift Keying (STSK) [43] and its 261 generalized form, namely GSTSK [54] was further extended by 262 applying SSK/SM to both the space and to the time dimensions 263 upon combining SSK/SM with space-time block codes, which 264 resulted in an improved diversity versus multiplexing tradeoff. 265 In contrast to the TA-index of conventional SM, in STSK [43], 266 the specific indices of the pre-designed space-time dispersion 267 matrices were exploited for conveying additional data. To be 268 specific, one out of $N_{t}$ dispersion matrices was activated rather 269 than simply activating one out of $N_{t}$ TAs in order to disperse a 270 PSK/QAM symbol in STSK, where a beneficial diversity gain 271 may be achieved as a merit of the simultaneous transmissions 272 from the multiple TAs. As a further advance, the STSK concept 273 was extended to the frequency domain in [55] and [56] with 274 the assistance of a Frequency-Shift Keying (FSK) modulator. 275 To be specific, in [55] the Space-Frequency Shift Keying 276 (SFSK) as well as the Space-Time-Frequency Shift Keying 277 (STFSK) schemes were proposed, which have the added benefit 278 of spreading the transmit signal across both the space and time 279 
TABLE I

CONTRIBUtion to SM SCHEME AND ITS RELATED VARIANTS

\begin{tabular}{|c|c|c|}
\hline Year & Authors & Contributions \\
\hline 2001 & "Chau and $\mathrm{Yu}[45]$ & $\begin{array}{l}\text { Introduced the concept of SM and exploited the distinct multipath fading } \\
\text { characteristics for antenna index detection. }\end{array}$ \\
\hline 2002 & Haas et al. $[42]$ & $\begin{array}{l}\text { Proposed an OSDM scheme, which uses Walsh-Hadamard codes and an antenna } \\
\text { array for data multiplexing. }\end{array}$ \\
\hline 2004 & Song et al. [62] & $\begin{array}{l}\text { Proposed channel hopping modulation, which is applicable to an arbitrary number } \\
\text { of TAs. }\end{array}$ \\
\hline 2006 & Mesleh et al. [63] & $\begin{array}{l}\text { Proposed an efficient MIMO scheme, namely SM, which maps multiple information } \\
\text { bits into a single information symbol and to the index of a single TA } \\
\text { transmitting antenna. }\end{array}$ \\
\hline \multirow[t]{3}{*}{2008} & Jeganathan et al. [46] & $\begin{array}{l}\text { Conceived an SSK concept and its improved version of the SSK modulation, } \\
\text { namely GSSK, which activates multiple TAs for data transmission. }\end{array}$ \\
\hline & Yang et al. [12] & $\begin{array}{l}\text { Introduced the IGCH technique based on the fact that the independent fading of } \\
\text { multiple channel can be used as an additional information channel. }\end{array}$ \\
\hline & Mesleh et al. [11] & $\begin{array}{l}\text { Proposed a simple MRC-based receiver design for SM, which detects the TA } \\
\text { index and APM separately. }\end{array}$ \\
\hline \multirow[t]{2}{*}{2009} & Abu-alhiga et al. [58] & $\begin{array}{l}\text { Designed a power-efficient SIM scheme, which maps a stream of bits into the indices } \\
\text { of the available subcarriers in an on-off keying fashion. }\end{array}$ \\
\hline & Jeganathan et al. [44] & $\begin{array}{l}\text { Presented the framework of SSK, which is a low-complexity version of SM } \\
\text { concept and exclusively employs the TA indices for data transmission. }\end{array}$ \\
\hline \multirow[t]{7}{*}{2010} & Di Renzo et al. [30] & $\begin{array}{l}\text { Introduced an opportunistic power allocation scheme for SSK modulation, which } \\
\text { exploits CSI for performance improvement }\end{array}$ \\
\hline & Mesleh et al. [51] & $\begin{array}{l}\text { Proposed a trellis coded SM (TC-SM) scheme, where the Trellis Coded Modulation } \\
\text { is applied to SM to improve its performance in correlated channels. }\end{array}$ \\
\hline & Serafimovski et al. [64] & $\begin{array}{l}\text { Introduced a Fractional Bit Encoded (FBE)-SM scheme, which allows the } \\
\text { transmitter to be equipped with an arbitrary number of TAs. }\end{array}$ \\
\hline & Fu et al. $[47]$ & $\begin{array}{l}\text { Proposed high-rate generalized SM, which uses multiple active TAs to encode } \\
\text { information bits. }\end{array}$ \\
\hline & Younis et al. [48] & $\begin{array}{l}\text { Proposed a GSM scheme, which sends the same symbol from more than one } \\
\text { transmit antenna at a time. }\end{array}$ \\
\hline & Sugiura et al. [43] & $\begin{array}{l}\text { A novel STSK modulation scheme is proposed, which constitutes a generalized shift } \\
\text { keying architecture utilizing both the space as well as time dimensions and hence } \\
\text { includes the SM and SSK schemes as special cases. }\end{array}$ \\
\hline & Renzo et al. [65] & $\begin{array}{l}\text { Introduced the Time-Orthogonal Signal Design assisted SM (TOSD-SM) for offering } \\
\text { transmit-diversity. }\end{array}$ \\
\hline \multirow[t]{6}{*}{2011} & Yang et al. [66] & $\begin{array}{l}\text { Designed a Bit-Padding IGCH (BP-IGCH) scheme, which eliminates the limitation } \\
\text { that the number of TAs has to be a power of two based on the IGCH concept. }\end{array}$ \\
\hline & Başar et al. $[50]$ & $\begin{array}{l}\text { Combined SM and STBC to take advantage of the benefits of both, while avoiding } \\
\text { their drawbacks. }\end{array}$ \\
\hline & Sugiura et al. $[54]$ & $\begin{array}{l}\text { Proposed a novel Generalized STSK (G-STSK) architecture for striking a flexible } \\
\text { tradeoff among diversity, throughput as well as complexity. }\end{array}$ \\
\hline & Ngo et al. [55] & $\begin{array}{l}\text { Proposed the SFSK modulation as well as the STFSK concept, which spreads the } \\
\text { transmit signal across the space- and time- and frequency-domain. }\end{array}$ \\
\hline & Qu et al. [67] & Conceived a block mapping SM (BMSM) scheme for increasing the transmit rate. \\
\hline & Başar et al. [52] & Proposed a new TC-SM scheme with for achieving higher diversity and coding gains. \\
\hline \multirow[t]{4}{*}{2012} & Zhang et al. [68] & $\begin{array}{l}\text { Introduced a novel SM scheme based on Ungerboeck's set partitioning for a } \\
\text { correlated Rician fading scenario. }\end{array}$ \\
\hline & Wang et al. $[49]$ & $\begin{array}{l}\text { Designed a novel high-rate Multiple Active-SM (MA-SM) schemes and a } \\
\text { near-optimal decoder with linear complexity. }\end{array}$ \\
\hline & Chang et al. $[69],[70]$ & $\begin{array}{l}\text { Proposed a new SSK modulation with Hamming code-aided constellation design for } \\
\text { striking a flexible tradeoff among transmission rate, performance and power. }\end{array}$ \\
\hline & Kuo $[71]$ & $\begin{array}{l}\text { Proposed a Symbol Coordinate Representations in Antenna Domains modulation } \\
\text {, which leads superior performance to both SM and GSSK at the same data rate. }\end{array}$ \\
\hline \multirow[t]{3}{*}{2013} & Di Renzo et al. [15] & $\begin{array}{l}\text { Illustrated the archived experimental results substantiating the benefits of SM } \\
\text { and presented its beneficial application areas. }\end{array}$ \\
\hline & Serafimovski et al. $[26]$ & First practical testbed implementation of SM in indoors (laboratory environment). \\
\hline & Younis et al. [25] & luation of SM in indoors using real-world measured channels. \\
\hline
\end{tabular}

280 domains, as well as the frequency domain. In [56], the STFSK 281 concept was extended to the Slow-Frequency-Hopping Multi282 ple Access (SFHMA) philosophy for the sake of supporting 283 multiple users and its Area Spectral Efficiency (ASE) gain over 284 the classic Gaussian Minimum Shift Keying (GMSK)-aided 285 SFHMA and GMSK assisted time-division/frequency-division 286 multiple access (TD/FDMA) systems was quantified.

287 Inspired by the concept of SM/SSK, the subcarrier orthogo288 nality can also be exploited and the indices of active subcarriers 289 of Orthogonal Frequency-Division Multiplexing (OFDM) [57] 290 symbols can be employed for conveying additional information, 291 which is referred to as Subcarrier-Index Modulation (SIM)
[58]. Based on the same principle, but following a different 292 approach from that of [58], a novel transmission scheme termed 293 as OFDM combined with Index Modulation (OFDM-IM) was 294 proposed in [59] for frequency selective fading channels, with 295 the objective of increasing the data rate as well as simultane- 296 ously improving the attainable BER performance. In Fig. 3, 297 we classify the above-mentioned schemes, which exploit dif- 298 ferent degrees of freedom offered by the temporal domain, 299 frequency domain and spatial domain fading. For completeness, 300 we also briefly allude to the classic time hopping impulse 301 modulation (THIM) [60], which exploits the indices of time- 302 slots for implicitly conveying additional data. As a further 303 


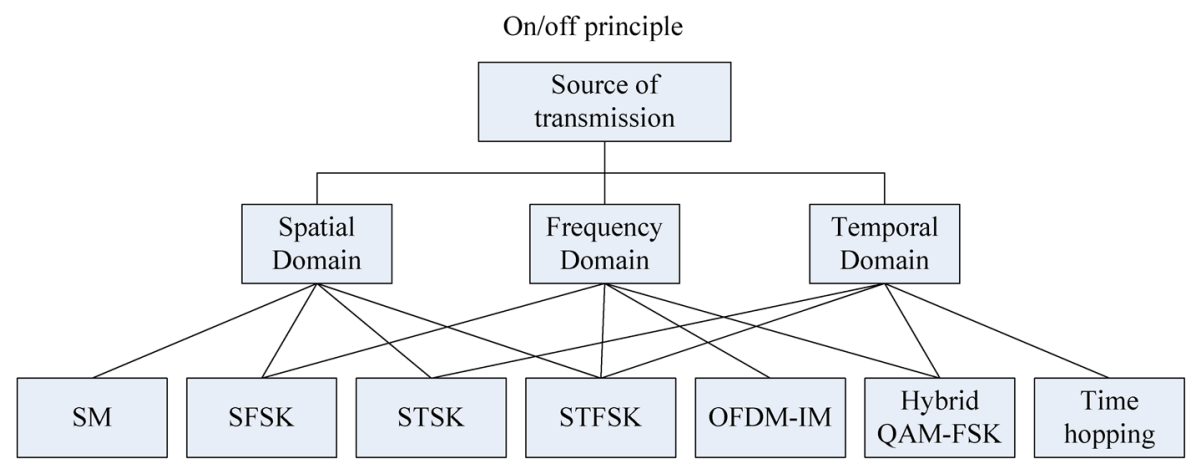

Fig. 3. Transmission techniques based on the on/off keying principle applied to the temporal domain, frequency domain and spatial domain. Here, we have "SM": spatial modulation, "SFSK": Space-Frequency Shift Keying, "STSK": Space-Time Shift Keying, "STFSK": Space-Time-Frequency Shift Keying, and "OFDM-IM": OFDM with Index Modulation.

304 improvement, hybrid QAM-FSK modulation [61] combine 305 the time-frequency domain for the sake of exploiting their 306 independent fading.

\section{C. Detector Design}

308 As seen in Fig. 2, the TA index is combined with the 309 APM symbol index by the SM mapper. Hence, only the TA 310 antenna index and the transmitted APM symbol index have to 311 be estimated at the receiver. Note that most variants of SM, such 312 as STSK and SSK, have an equivalent system model similar to 313 (1), which is free from the effects of ICI, and each equivalent 314 transmit vector includes only a single non-zero component [43], 315 [44]. As a result, they may be able to use the same detection 316 algorithm. As indicated in [72]-[88], the detection techniques 317 of SM-MIMO systems may be broadly divided into four funda318 mental categories: Maximum Likelihood (ML) detection [72]319 [74], Matched Filter (MF) based detection [11], [75], Sphere 320 Decoding (SD) algorithm based detection [76]-[79] and hybrid 321 detection, which combines the modified MF concept and the 322 reduced-complexity exhaustive ML search of [12], [80]-[88]. 323 An overview of the various detection techniques conceived 324 for SM-related schemes is seen in Fig. 4. Next, they will be 325 characterized in more detail.

326 An optimal ML-based SM detector, which carries out an 327 exhaustive search for the global optimum in the entire signal 328 space, was developed in [72]. This detector jointly detects the 329 active TA index as well as the transmitted APM symbol and 330 then retrieves the original data bit sequence. In [73], the authors 331 have derived a soft-output ML detector for recovering the 332 desired signals with the aid of soft decisions, and have shown 333 that the soft-output ML detector outperforms its hard-decision 334 counterpart. Moreover, in [74], the authors have exploited the 335 inherent ML data detection in the context of STSK systems 336 and proposed a semi-blind iterative channel estimation and 337 data detection scheme for STSK, which is capable of reducing 338 the training overhead required. Furthermore, a low-complexity 339 multi-stage ML detector was proposed for the ICGH of [12], 340 which adopts the principles of SM. The proposed detector 341 estimates the APM symbol prior to detecting the TA index. 342 Unlike the ML detector of other spatial multiplexing MIMO 343 techniques, the complexity of the single-stream ML receiver 344 only increases linearly with the number of TAs. However, as

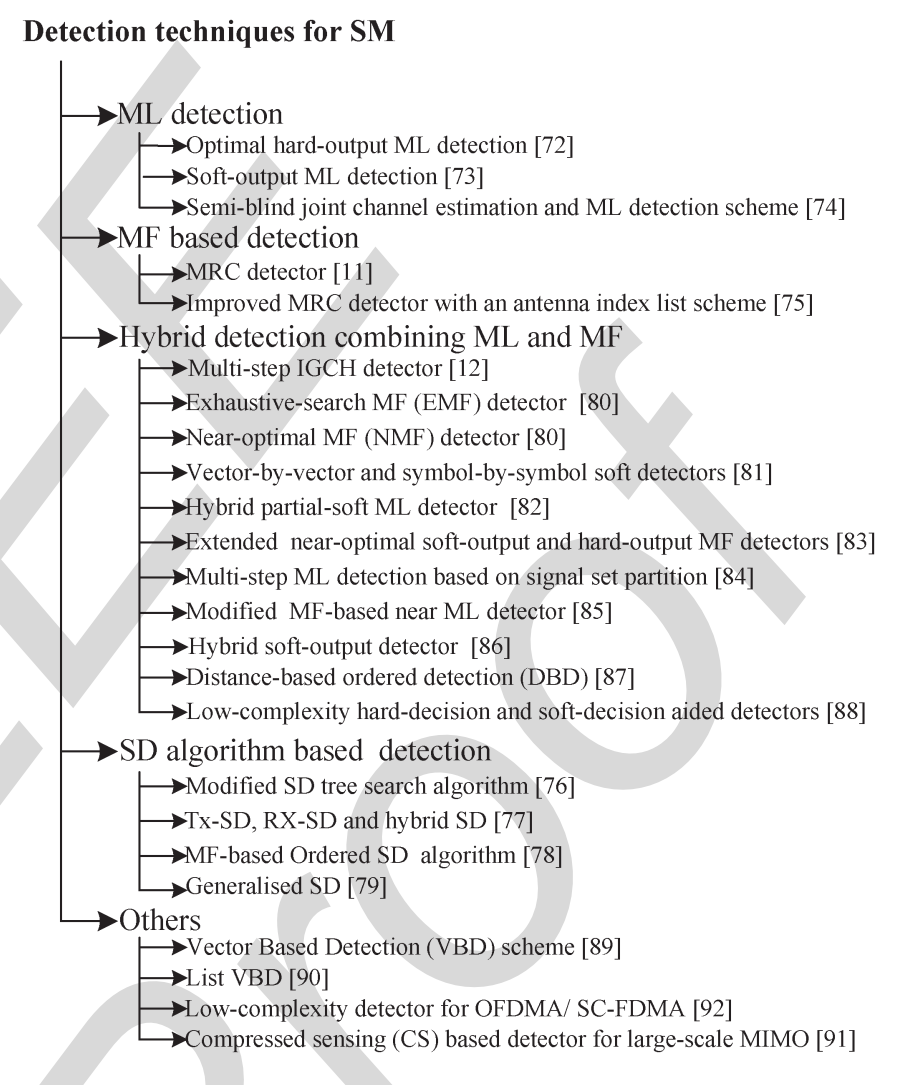

Fig. 4. Overview of SM detectors and related techniques.

the transmission rate increases, even the complexity of the ML 345 single-stream detector might become excessive. 346

Among the promising alternatives, the MF-based detector 347 exhibits a considerably reduced complexity, since the activated 348 TA index and the modulated APM constellation point are sep- 349 arately estimated. However, as mentioned in [11], the conven- 350 tional MF detector, namely the MRC, only performs well under 351 the idealized assumption of perfect channel knowledge. This 352 detector was improved in [75] and a TA index list based scheme 353 was introduced for all the conventional MIMO channels. 354

For the sake of approaching the single-stream ML detector's 355 performance without any substantial performance degradation, 356 beneficial hybrid detectors were designed for the SM family 357 in [80]-[88], which combine the modified MF concept of [11] 358 and the reduced-complexity exhaustive ML search philosophy 359 
360 of [72]. For example, in [80], two modified MF-based detec361 tors, namely the Exhaustive-search based MF (EMF) detector 362 and the Near-optimal MF (NMF) detector were proposed for 363 achieving a better performance than the conventional MF detec364 tor. However, the EMF has to invoke an exhaustive signal space 365 search at the MF's output for maintaining the ML's perfor366 mance, which prevents the detector from achieving a significant 367 reduction in complexity, when high data rates are required. By 368 contrast, the NMF detector further reduced the EMF's complex369 ity, but naturally it performs worse than the ML detector [72]. 370 To overcome this limitation, the authors of [83] proposed an 371 extended NMF detector, which relies on finding multiple high372 probability indices for the sake of attaining further performance 373 improvements. Then, this improved NMF detector was further 374 simplified in [87] and [88]. Considering that SM-MIMO sys375 tems typically rely on powerful channel codes, an attractive 376 detector has to provide soft-decision-based information. In [44] 377 and [73], an optimal Maximum a Posteriori (MAP) detector 378 was invoked for turbo-coded SM schemes. However, it suffers 379 from the problem of having a high complexity. In [81], the au380 thors have proposed a low-complexity vector-by-vector based 381 soft-detector operating on a symbol-by-symbol basis, where the 382 associated complexity was considerably reduced compared to 383 that of the max-log MAP detector's, albeit this was achieved at 384 the cost of a modest performance degradation.

385 On the other hand, the SD [93], [94], which is widely used 386 in spatial multiplexing systems, avoids the exhaustive search 387 of the potentially excessive-complexity signal constellation by 388 examining only those candidate solutions that lie inside an 389 SNR-dependent decoding sphere. However, the conventional 390 SD and the more advanced SD methods [94] are oblivious of the 391 specific principle of SM, namely that only a single TA is active 392 at any given time instant. As a result, the SD methods designed 393 for spatial multiplexing MIMOs cannot be directly applied 394 to SM-MIMO detection. In [76], a modified SD algorithm 395 referred to as SM-SD was proposed, which is based on the tree396 search structure. The SM-SD algorithm exploits the specific 397 transmission mode of SM and hence attains a considerable 398 complexity reduction. However, the performance of the SM399 SD algorithm depends on the particular choice of the SNR400 dependent initial search-radius as well as on the transmitter 401 parameters. Hence, in [78], an Ordered SD (OSD) algorithm 402 was proposed for the family of SM arrangements for the sake 403 of reducing the receiver's complexity, while maintaining the op404 timum single-stream ML performance, which searches through 405 the signal space sequentially according to the sorted TA set. 406 Recently, a generalized version of the SM-SD was proposed in 407 [77] and [79].

408 Relying on a novel approach, in [89] the authors have pro409 posed a new Vector Based Detection (VBD) scheme for SM, 410 which is suitable for high-order APM constellations. In [90], 411 an improved VBD scheme, namely the list-VBD was proposed, 412 where the TA index detection is performed first and a list of the 413 best candidates survives. As indicated in Section I, the family 414 of SM constitutes an attractive framework for the emerging 415 family of large-scale MIMO systems in reducing the hard416 ware costs and detection complexity, which becomes realistic 417 at microwave frequencies. Since ML detection of high-order
APM schemes in large-scale high-rate MIMO systems has 418 a potentially excessive complexity, in [91] a low-complexity 419 Compressed Sensing (CS) based detector was proposed for 420 overcoming this problem by exploiting the sparsity in SM 421 signaling. Again, the family of SM has also been effectively 422 extended to the Orthogonal Frequency Division Multiple Ac- 423 cess (OFDMA)/Single-Carrier Frequency Division Multiple 424 Access (SC-FDMA)-aided architecture and some related low- 425 complexity detectors were proposed in [92].

426

Additionally, most of the above-mentioned detectors assume 427 that perfect CSI is available at the receiver. However, it is chal- 428 lenging to acquire accurate CSI in high-speed vehicles and mul- 429 tiple antenna systems. In order to dispense with CSI-estimation, 430 the class of Differentially-encoded STC (DSTC) was proposed 431 in [95] and [96]. Specifically, the Unitary Space-Time Modula- 432 tion (USTM) scheme does not require CSI estimation and hence 433 facilitates non-coherent detection at the receiver. Motivated by 434 the concept of DSTC, the design of non-coherent SM-MIMO 435 schemes was investigated in [43], [97], and [98]. To be specific, 436 in [43], the differential STSK (DSTSK) concept was proposed 437 with the aid of the Cayley unitary transformation, which has 438 a low-complexity single-stream non-coherent detector. In [97], 439 the DSTSK scheme was further developed for the sake of avoid- 440 ing the nonlinear Cayley transform and a reduced-complexity 441 multiple-symbol differential sphere detector was proposed for 442 rapidly fading channels. Moreover, a PSK-aided differential 443 modulation concept was conceived in [98], which relies on 444 differential decoding while retaining the fundamental benefits 445 of coherent SM-MIMO schemes.

D. Channel Capacity and Error Performance Metric

1) Channel Capacity: The capacity of SM constitutes a vi- 448 tally important research topic. In [12], the authors have derived 449 the capacity of SM in the context of Rayleigh fading chan- 450 nels, assuming continuous-amplitude discrete-time Gaussian 451 distributed transmitted signals. This capacity is also referred to 452 as the Continuous-input Continuous-output Memoryless Chan- 453 nel (CCMC) capacity [7]. However, this assumption cannot be 454 readily satisfied in a practical communication system, unless 455 carefully designed superposition modulation is used [99]. By 456 contrast, in [43] the Discrete-input Continuous-output Memo- 457 ryless Channel (DCMC) capacity [100] of the family of SM 458 scheme was formulated, where the transmitted signals were 459 drawn from finite-alphabet discrete constellations, such as the 460 classic APM schemes [13]. Moreover, a closed-form expression 461 of the mutual information of SM based Multiple-Input Single- 462 Output (MISO) channels was derived and the impact of finite- 463 alphabet inputs on the attainable performance of SM was in- 464 vestigated in [101]. Owing to its particular operating principle, 465 its capacity and the corresponding optimization algorithms still 466 require further research.

467

Fig. 5 shows the CCMC and DCMC capacity curves of the 468 $(4 \times 2)$-element SM-MIMO scheme. Furthermore, the G4- 469 STBC arrangement of [3] was also considered as benchmarkers 470 in Fig. 5. As shown in Fig. 5, the CCMC capacity of the SM 471 scheme is higher than that of G4-STBC. Additionally, observe 472 in Fig. 5 that the DCMC capacity tends to be increased upon 473 


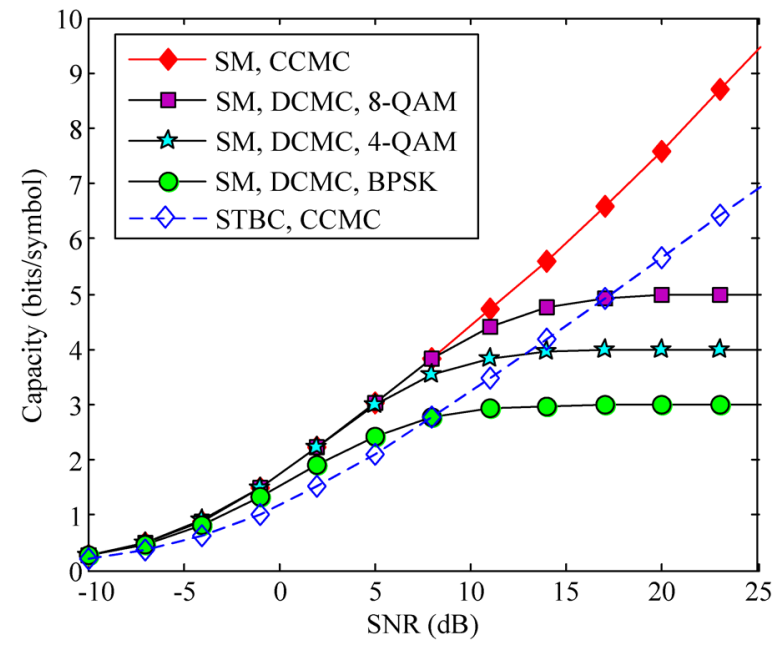

Fig. 5. Bandwidth efficiency of $(4 \times 2)$-element SM system, comparing the CCMC and the DCMC capacity.

474 increasing the modulation order, as noted in [12]. Moreover, as 475 indicated in [8] and [26], the capacity of SM may be lower than 476 that of the V-BLAST arrangement, however its detection com477 plexity does not depend on the number of transmit antennas. 478 This attractive advantage facilitates the practical application of 479 SM-MIMO.

480 2) Error Performance Metric: The BER performance of SM 481 has also been studied extensively in the context of various 482 channel models and MIMO setups [28]-[34]. Generally, the an483 alytical study of SM-MIMO systems tends to rely on its union 484 bound based approximation [102]. However, apart from the 485 STSK studies of [43] and the investigations of Di Renzo et al. 486 [15], the studies in [27], [28], and [32]-[34] considered the sim487 plified version of SM, namely SSK. For the conventional SM 488 combining SSK with classic APM techniques for the sake of 489 transmitting additional bits, the analytical studies disseminated 490 in [11], [14], [29], and [103] exploited some of the fundamental 491 properties of SM related to the channel's correlation, to its 492 transmit diversity, channel estimation errors and coding gain. 493 For example, in [103] the authors have provided a closed-form 494 Average Bit Error Probability (ABEP) upper bound expression 495 based on the conventional union-bound methods, which also 496 quantified the transmit diversity order of SM. This framework 497 is usually used as a reference for highlighting the advantages 498 of SM over other MIMO arrangements, such as the classic 499 STBC and VBLAST schemes. In [29], an improved union500 bound is formulated, which partitions the ABEP expression of 501 SM-MIMO systems into three terms: the term $P_{\text {spatial }}(\rho)$ only 502 related to the spatial signals (i.e., TA index), the term $P_{\text {signal }}(\rho)$ 503 is only related to the APM signals, while the joint term $P_{\text {joint }}(\rho)$ 504 depends on both the spatial signals and on the APM signals, 505 where $\rho$ is the average SNR. This bound is formulated as

$$
\mathrm{P}_{\mathrm{SM}}(\rho) \leq P_{\text {spatial }}(\rho)+P_{\text {signal }}(\rho)+P_{\text {joint }}(\rho)
$$

506 Assuming i.i.d. Rayleigh fading channels, $P_{\text {signal }}(\rho)$ pre507 dominantly depends on the minimum ED $d_{\text {min }}$ of the constel508 lation points of APM, while $P_{\text {joint }}(\rho)$ and $P_{\text {spatial }}(\rho)$ mainly 509 depend on the modulus values $\beta_{l}(l=1, \cdots, L)$ of the APM

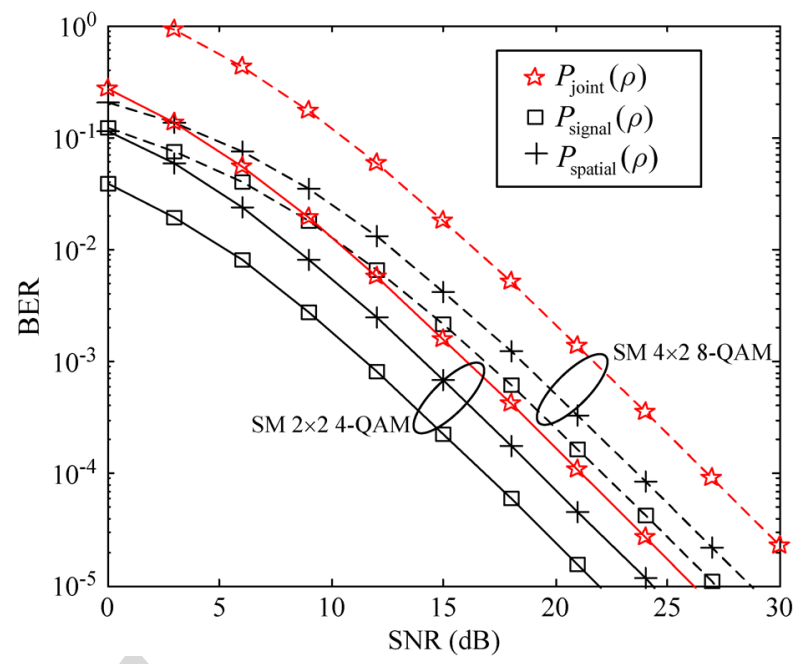

Fig. 6. The ABEPs of SM-MIMO: $P_{\text {signal }}(\rho), P_{\text {joint }}(\rho)$ and $P_{\text {spatial }}(\rho)$.

constellation points, as detailed in [29]. As a result, $\mathrm{P}_{\mathrm{SM}}(\rho) 510$ of (2) depends both on the minimum ED of the specific APM 511 constellations employed, as well as on the absolute values of 512 the APM-symbols. This improved ABEP upper bound of SM 513 provides deeper insights into the interactions of the APM signal 514 constellation and the spatial signal constellation. For example, 515 the interaction term $P_{\text {joint }}(\rho)$ of (2) dominates the performance 516 of SM in diverse popular MIMO configurations, as indicated in 517 Fig. 6. On the other hand, it can also be used for optimizing the 518 system's performance by exploiting any statistical knowledge 519 about the Channel State Information (CSI) at the transmitter 520 and we will discuss in Section III.

Moreover, since the exact ABEP does not have a simple 522 closed form solution, the nearest neighbor approximation was 523 proposed in [104]. Assuming that all the channel inputs are 524 equally likely, the nearest neighbor approximation of the Pair- 525 wise Error Probability (PEP) for a given channel matrix $\mathbf{H}$ can 526 be expressed as [105]

$$
P_{e \mid \mathbf{H}} \approx \lambda \cdot Q\left(\sqrt{\frac{1}{2 N_{0}} d_{\min }^{2}(\mathbf{H})}\right)
$$

where we have $Q(x)=(1 / \sqrt{2 \pi}) \int_{x}^{\infty} e^{-y^{2} / 2} d y$, and $\lambda$ is the 528 number of neighboring constellation points [10] associated with 529 the free distance $(\mathrm{FD}) d_{\min }(\mathbf{H})$ defined as

$$
d_{\min }(\mathbf{H})=\min _{\substack{\mathbf{x}_{i}, \mathbf{x}_{j} \in \mathbb{X}, \mathbf{x}_{i} \neq \mathbf{x}_{j}}}\left\|\mathbf{H P}\left(\mathbf{x}_{i}-\mathbf{x}_{j}\right)\right\|
$$

where $\mathbb{X}$ is the set of legitimate transmit symbols, while $\mathbf{x}_{i} 531$ and $\mathbf{x}_{j}$ are two distinct transmitted symbols in $\mathbb{X}$. In (4), $\mathbf{P}$ is 532 the transmit preprocessing (TPP) matrix, which is the $\left(N_{t} \times 533\right.$ $N_{t}$ )-element identity matrix I for conventional open-loop SM 534 schemes dispensing with TPP.

Note that the nearest neighbor approximation of the PEP will 536 always be slightly lower than that provided by the union bound, 537 since this approximation does not include the errors associated 538 with those legitimate symbols that are farther apart than the 539 FD. However, in case of low SNRs, there is a non-negligible 540 probability of corrupting a symbol into more distant symbols. 541 
542 Nonetheless, the result is quite close to the exact probability of 543 symbol error at high SNRs, as detailed in [105]. Indeed, since 544 the error events mainly arise from the nearest neighbors, the 545 maximization of the FD in (3) directly reduces the probability 546 of error, especially at high SNRs [106]. As a result, the bound 547 of (3) can be adapted for system optimization by exploiting the 548 knowledge of the near-instantaneous CSI, as discussed these in 549 more detail in Section IV.

550 Furthermore, the effects of CSI errors on the achievable per551 formance of SM-MIMOs were further researched in [34] and 552 [107]-[109]. It was found that SM is quite robust to imperfect 553 CSI compared to V-BLAST. For example, in [107] an asymp554 totically tight upper bound on the ABEP was derived for SM 555 under imperfect CSI and the simulation results confirmed that $556 \mathrm{SM}$ is more robust to channel estimation errors than V-BLAST 557 for reasonable practical channel estimation error values.

\section{8 \\ III. APM Constellation Optimization}

559 As indicated in (2), the performance of SM-MIMO systems 560 is highly dependent on the specific APM signal constellation 561 adopted. In a conventional Single-Input Single-Output (SISO) 562 system, the Gray-coded Maximum-minimum distance (MMD) 563 QAM constellation minimizes the Bit Error Ratio (BER) [13]. 564 However, the advantage of MMD-QAM may be eroded in SM565 MIMO systems [29]. This is due to the fact that the BER 566 performance of SM-MIMO systems is jointly determined by 567 the spatial signal (i.e., TA indices), by the classic APM con568 stellation and by their interaction [29]. Hence, a suitable APM 569 scheme has to be designed for this hybrid modulation scheme. 570 Furthermore, SM also allows us to achieve a high transmis571 sion rate by combining its benefits with those of the classic 572 APM schemes, as detailed in [46]-[49]. However, when the 573 source employs higher-order square QAM in order to increase 574 the attainable transmission rate, a high Peak-to-Average-Power 575 Ratio (PAPR) [110] is encountered, hence requiring a low576 efficiency linear power amplifier [111]. To overcome this im577 pediment, peak-power reduction constellation shaping [110] 578 may be employed at the transmitter, albeit this technique im579 poses additional complexity. Thus, for the sake of achieving a 580 high power-efficiency, the choice of the modulation scheme in 581 SM-MIMO systems has to be revisited.

582 The effects of APM schemes on the performance of SM 583 have been investigated in [112]-[114]. More specifically, in 584 [112], the dispersion matrices and the signal constellations were 585 jointly optimized for a near-capacity precoded STSK system, 586 which includes SM as a special case and strikes a flexible rate587 versus-diversity tradeoff. It was also shown in [80] that the star588 QAM aided STSK scheme outperforms its MMD based square589 QAM aided counterpart. This is because the STSK's achievable 590 performance depends both on the minimum ED of the APM 591 constellation employed, as well as on the absolute values of the 592 modulated symbols, which may also be valid for SM systems, 593 as shown in (2) [29]. More recently, in [31] low-complexity, 594 yet single-stream ML transmit diversity schemes have been 595 studied by analyzing the impact of the spatial constellation and 596 shaping filters. In [70], a Hamming code construction technique 597 was proposed as a modulation design strategy for SSK-based

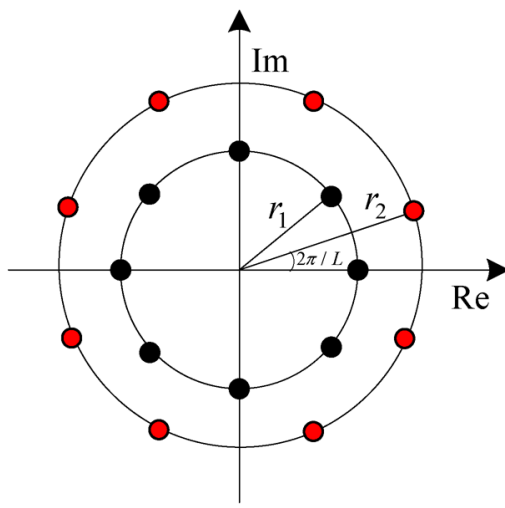

Fig. 7. The complex signal constellation of 16-ary star-QAM. The symbols are evenly distributed on two rings, and the phase differences between the neighboring symbols on the same ring are equal.

systems for the sake of improving their error probability. In 598 [113], a new SM constellation design strategy was proposed 599 based on the ED of the constellation, which retains the key 600 advantages of SM, while activating multiple TAs. In [114], 601 two approaches were investigated with the goal of designing 602 the SSK's transmit constellation space by relying either on 603 the idealized simplifying assumption of having perfect CSI or 604 on the more practical scenario of imperfect CSI at the trans- 605 mitter, in order to increase the distance between each pair of 606 the received combined TA-APM vector. The above-mentioned 607 techniques were however mainly conceived for STSK and SSK 608 schemes, but may not be readily applicable to the conventional 609 SM scheme.

610

In [29], the performance of SM systems relying both on 611 conventional QAM and PSK modulation were studied, demon- 612 strating that in some MIMO setups, the PSK-modulated SM 613 scheme may outperform the identical-throughput MMD-QAM 614 SM scheme. More specifically, as shown in [29] and [115], for 615 certain SM-MIMO configurations, $P_{\text {signal }}(\rho)$ of (2) is signifi- 616 cantly higher than the sum of $P_{\text {joint }}(\rho)$ and $P_{\text {spatial }}(\rho)$, which 617 implies that the minimum ED of APM constellations dominates 618 the performance of SM. In this scenario, MMD-QAM may 619 constitute an attractive APM candidate for minimizing the 620 ABEP. By contrast, as shown in Fig. 6, if $P_{\text {signal }}(\rho)$ is lower 621 than the sum of $P_{\text {joint }}(\rho)$ and $P_{\text {spatial }}(\rho)$, which implies that 622 the moduli of the APM constellation points dominates the 623 $\mathrm{P}_{\mathrm{SM}}(\rho)$ term, then a constant-modulus modulation scheme, 624 such as PSK, may be optimal, as indicated in [29]. Recall that 625 $P_{\text {signal }}(\rho)$ of (2) is dominated by the minimum ED $d_{\text {min }}$, while 626 $P_{\text {joint }}(\rho)$ and $P_{\text {spatial }}(\rho)$ mainly depend on the modulus values 627 $\beta_{l}(l=1, \cdots, L)$ of the APM constellation adopted. Note that 628 the modulus values $\beta_{l}(l=1, \cdots, L)$ are represented by the 629 Frobenius norms of the APM constellation points. These results 630 suggested that for the sake of jointly minimizing $P_{\text {signal }}(\rho), 631$ $P_{\text {joint }}(\rho)$ and $P_{\text {spatial }}(\rho)$ of (4), we can readily focus our 632 attention on design of $d_{\min }$ and on the $\beta_{l}$ parameters of APM. 633

On the other hand, star-QAM [13] constitutes a special case 634 of circular APM, which is capable of outperforming the classic 635 square-shaped QAM constellation in peak-power-limited sys- 636 tems. Hence its diverse relatives have been adopted in most 637 of the recent satellite communication standards, such as the 638 
TABLE II

The Minimum ED of DifFERENT APM SCHEMES

\begin{tabular}{|l|c|c|c|c|c|}
\hline Modulation order $(L)$ & 2 & 4 & 8 & 16 & 32 \\
\hline PSK & $d_{\min }=2$ & $d_{\min }=\sqrt{2}$ & $d_{\min }=0.76$ & $d_{\min }=0.39$ & $d_{\min }=0.19$ \\
\hline QAM & -- & $d_{\min }=\sqrt{2}$ & $d_{\min }=0.81$ & $d_{\min }=0.63$ & $d_{\min }=0.41$ \\
\hline Star-QAM & $d_{\min }=2$ & $d_{\min }=\sqrt{2}$ & $d_{\min }=0.91$ & $d_{\min }=0.57$ & $d_{\min }=0.40$ \\
\hline
\end{tabular}

639 Digital Video Broadcast System (DVB) S2, DVB-SH, as well 640 as in the Internet Protocol over Satellite (IPOS) and Advanced 641 Broadcasting System via Satellite (ABS-S) [116]. To elaborate 642 a little further, the star-QAM constellation is composed of 643 multiple concentric circles and it was shown to be beneficial in 644 the context of STSK systems [80]. However, the constellations' 645 optimization has not been carried out for star-QAM aided SM. 646 In order to make the choice of the APM parameters $d_{\text {min }}$ 647 and $\beta_{l}$ as flexible as possible, we consider a class of star648 QAM constellations, which subsumes the classic PSK as a 649 special case, but may also be configured for maximizing the 650 minimum ED of the constellation by appropriately adjusting 651 the ring ratios of the amplitude levels. For the sake of sim652 plicity, we consider the example of a twin-ring 16-star-QAM 653 constellation having a ring-ratio of $\alpha=r_{2} / r_{1}$ as shown in 654 Fig. 7. The symbols are evenly distributed on the two rings 655 and the phase differences between the neighboring symbols 656 on the same ring are equal. Unlike the conventional twin-ring 657 star-QAM constellation [116], the constellation points on the 658 outer circle of star-QAM constellation are rotated by $2 \pi / L$ 659 degrees compared to the corresponding constellation points on 660 the inner circle. Hence again, the conventional PSK constitutes 661 an integral part of our star-QAM scheme, which is associated 662 with a ring-ratio of $\alpha=1$. Note that although this twin-ring 663 star-QAM constellation has indeed been invoked for nonco664 herent detection [117], it has not been considered whether 665 this constellation can be directly applied to SM for achieving 666 performance improvements.

667 Table II summarizes the minimum EDs $d_{\min }$ between the 668 constellation points for different APM schemes, where the 669 modulation order is the number of the constellation points. 670 Moreover, the $L$-PSK $/ L$-QAM schemes in [13] are used. It is 671 shown that the star-QAM is capable of achieving almost the 672 same minimum ED as the MMD-based QAM [8].

673 Given an $\left(N_{r} \times N_{t}\right)$-element MIMO setup having a trans674 mission rate of $m_{\text {all }}$, and $L$ modulation levels, the goal of star675 QAM aided signaling constellation optimization is to find the 676 ring-ratio $\alpha$, which minimizes the ABEP of SM-MIMO of (2). 677 Following this approach, the related optimization problem may 678 be formulated as

$$
\left\{\begin{array}{c}
\alpha^{*}=\min _{\alpha} \mathrm{P}_{\mathrm{SM}}(\rho) \\
\text { s.t. } \alpha \geq 1
\end{array}\right.
$$

679 Based on an exhaustive numerical search, for example, for 680 the 16-star-QAM aided $(4 \times 4)$-element SM-MIMO, the opti$681 \mathrm{mal}$ ring ratio was found to be $\alpha^{*}=1.7$ [118]. According to (2), 682 this optimized star-QAM aided SM scheme provides an SNR 683 gain of about $3 \mathrm{~dB}$ over the conventional 16-PSK modulated 684 SM scheme and an SNR gain of about $1.1 \mathrm{~dB}$ over the identical685 throughput Gray-coded MMD 16-QAM modulated SM scheme 686 at $\mathrm{BER}=10^{-5}$. Note that the optimized star-QAM constella-

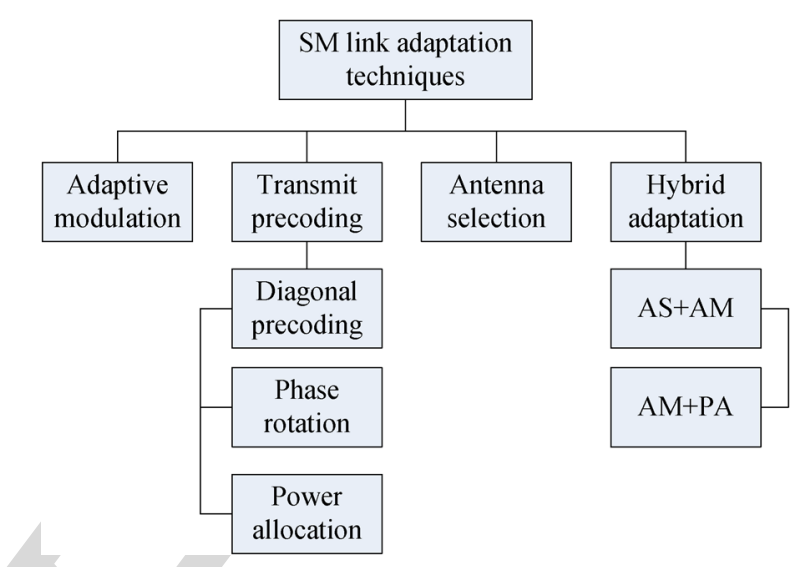

Fig. 8. Classification of the LA techniques designed for SM-MIMO. Here, AS+AM: antenna selection combined with adaptive modulation, AM+PA: adaptive modulation combined with power allocation.

tion can be designed off-line based on the CSI statistics (i.e., 687 the fading type) for different SM-MIMO systems and hence 688 the resultant system does not need any feedback. Next, we will 689 introduce a suite of beneficial adaptation techniques based on 690 the assumption that the knowledge of the near-instantaneous 691 channel matrix is available at the receiver in the frequency flat- 692 fading channel.

\section{Link ADAPTATION TECHNiQUES}

Link Adaptation (LA) has an important role in wireless 695 communication systems [39]-[41]. Traditionally, LA refers to 696 the concept of dynamically adjusting the transmit parameters, 697 such as the modulation order and coding rate according to the 698 near-instantaneous channel conditions. LA has been extensively 699 studied in the conventional MIMO context for the sake of im- 700 proving the achievable multiplexing and diversity performance. 701 However, it has not been considered, whether these existing LA 702 techniques can be directly applied to SM-based transmission 703 systems. Note that the introduction of LA techniques in SM- 704 MIMO should not jeopardize the advantages of SM, such as the 705 avoidance of the IAI, IAS and multiple RF chains [11]. This 706 makes the design of LA algorithms more challenging. In order 707 to increase the robustness of the SM-MIMO system, several 708 limited-feedback aided LA techniques have been proposed in 709 [30], [104], [115], and [119]-[130], as summarized in Fig. 8. 710 Depending on the MIMO scheme's degree freedom, these 711 techniques can be roughly divided into four types, namely into 712 Adaptive Modulation (AM) [104], [115], [119], [120], transmit 713 precoding (TPC) [30], [103], [121]-[125], Antenna Selection 714 (AS) [126]-[128] and Hybrid Adaptation (HA) techniques re- 715 lying on diverse combinations of the above three [115], [129], 716 [130], as shown in Fig. 8. To elaborate a little further, the 717 


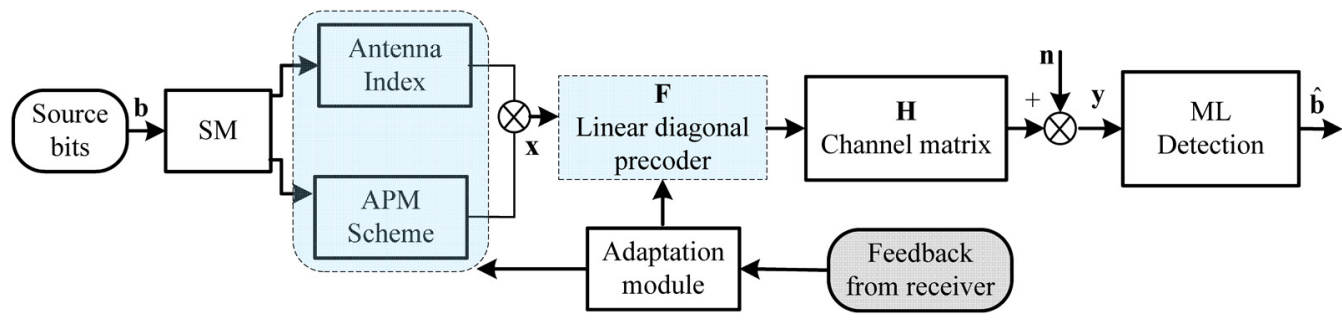

Fig. 9. Block diagram of LA-aided MIMO communication systems.

718 general philosophy of a LA-aided SM-MIMO system obeying 719 the architecture of Fig. 9 can be summarized as follows.

720 Algorithm 2: The adaptation process of LA-aided SM721 MIMO systems

1) Consider an $\left(N_{r} \times N_{t}\right)$-element SM-MIMO system associated with the transmission rate $m_{\text {all }}$;

2) The receiver estimates the CSI and decides upon the optimum transmit mode, which is then sent back to the transmitter through a low-rate feedback channel;

3) The transmitter processes the feedback information and employs the optimum transmission mode (i.e., the modulation orders and the precoding matrix) for its transmission.

731 Having formulated the SM-MIMO's LA algorithm, let us 732 now describe the class of LA techniques with the aid of 733 Fig. 8 developed for the family of SM-MIMO schemes in more 734 detail below. Note that in this treatise only the TPC matrix $735 \mathbf{P}$ and the transmit symbol $\mathbf{x}$ are adapted in response to the 736 near-instantaneous channel conditions in order to improve the 737 system's performance, as indicated in (4).

\section{A. Adaptive Modulation}

739 Again, AM techniques are capable of alleviating the adverse 740 effects of channel fading, so as to achieve an increased data 741 rate or a reduced BER [131], which have hence been adopted 742 in most of the recent communication standards, such as 3GPP, 743 3GPP2, IEEE 802.11a, IEEE 802.15.3 and IEEE 802.16 [132]. 744 SM may also be beneficially combined with AM for adjust745 ing the transmission parameters for the sake of accommodating 746 time-varying channels. Therefore, the beneficial combination of $747 \mathrm{AM}$ and SM-MIMO techniques is a promising design alterna748 tive for high-rate wireless systems.

749 To this end, adaptive SM-MIMO architectures relying on 750 different combinations of modulation/coding schemes were 751 proposed in [120], which aimed for maximizing the channel 752 capacity at a predefined target BER, rather than for optimizing 753 the BER. By contrast, in [104] a near-instantaneously Adaptive 754 SM (ASM) scheme was proposed for improving the attainable 755 system performance, while maintaining a fixed average transmit 756 rate with the aid of AM techniques. In ASM, the receiver 757 requests the most suitable modulation order to be used by 758 the transmitter for each TA and/or time-slot. Assuming that 759 no-transmission, BPSK and $M$-QAM are available for each
TA, which are represented by the set $\mathbb{M}_{\text {all }}$, the detailed design 760 procedure of ASM schemes can be summarized as follows: 761

Algorithm 3: Adaptive SM

1) Given the transmit parameters as: $N_{t}, N_{r}$ and the trans- 763 mission rate $m_{\text {all }}$, generate all the legitimate modulation 764 order combinations for a given $m_{\text {all }}$ and represent these 765 combinations as a set $\mathbf{R}=\left\{\mathbf{r}_{1}, \mathbf{r}_{2}, \cdots, \mathbf{r}_{j}, \cdots, \mathbf{r}_{J}\right\}, 766$ where we have $\mathbf{r}_{j}=\left[r_{j}^{1}, \cdots, r_{j}^{n} \cdots, r_{j}^{N_{t}}\right]$ and $r_{j}^{n}$ denotes 767 the modulation order for the $n$th $\left(n=1,2, \cdots N_{t}\right)$ TA of 768 the $j$ th ASM combination.

769

2) Based on the optimization rule, such as the nearest neigh- 770 bor approximation of (3), we can achieve a performance 771 gain by maximizing $d_{\min }(\mathbf{H})$ with the aid of switching 772 among these candidates.

773

3) Then, the corresponding index of the optimal ASM mode 774 is fed back to the transmitter, which transmits the symbols 775 accordingly.

776

In (3), the conditioned PEP is a monotonically decreasing 777 function of $d_{\min }(\mathbf{H})$. Hence, the attainable system performance 778 can be improved by maximizing the FD $d_{\min }(\mathbf{H})$ by adapting 779 the transmit parameters. As an example, let us consider a 780 $(2 \times 2)$-element SM-MIMO transmission scheme associated 781 with $m_{\text {all }}=3$ bits/symbol under a channel realization matrix 782 $\mathbf{H}$, which is given by

$$
\mathbf{H}=\left[\begin{array}{cc}
0.26-0.75 \mathrm{i} & 1.33+0.49 \mathrm{i} \\
0.03+1.30 \mathrm{i} & -0.61+0.25 \mathrm{i}
\end{array}\right]
$$

Let us assume that no-transmission, BPSK, QPSK, 8-QAM, 784 16-QAM, 32-QAM and 64-QAM are available for each TA and 785 these schemes are represented as $\mathbb{M}_{\text {all }}=\{0,2,4,8,16,32,64\}$, 786 where the no-transmission mode has the identifier of $M=0,787$ while the BPSK and QPSK constellations are denoted as $M=2788$ and $M=4$ respectively. For $m_{\text {all }}=3$ bits/symbol, we have five 789 ASM mode candidates denoted as $\mathbf{R}=\left\{\mathbf{r}_{1}, \mathbf{r}_{2}, \mathbf{r}_{3}, \mathbf{r}_{4}, \mathbf{r}_{5}\right\}=790$ $\{[16,0],[2,8],[4,4],[8,2],[0,16]\}$, where $\mathbf{r}_{1}=[16,0]$ repre- 791 sents that 16-QAM and no-transmission are assigned to the 792 first and the second TA, respectively, while the candidate [4,4] 793 corresponds to the conventional non-adaptive SM scheme using 794 QPSK for both TAs.

Based on Algorithm 3, Fig. 10 shows the detailed actions of 796 the ASM scheme for this 3-bits/channel-use system. As shown 797 in Fig. 10, the five ASM modes (the legitimate modulation 798 


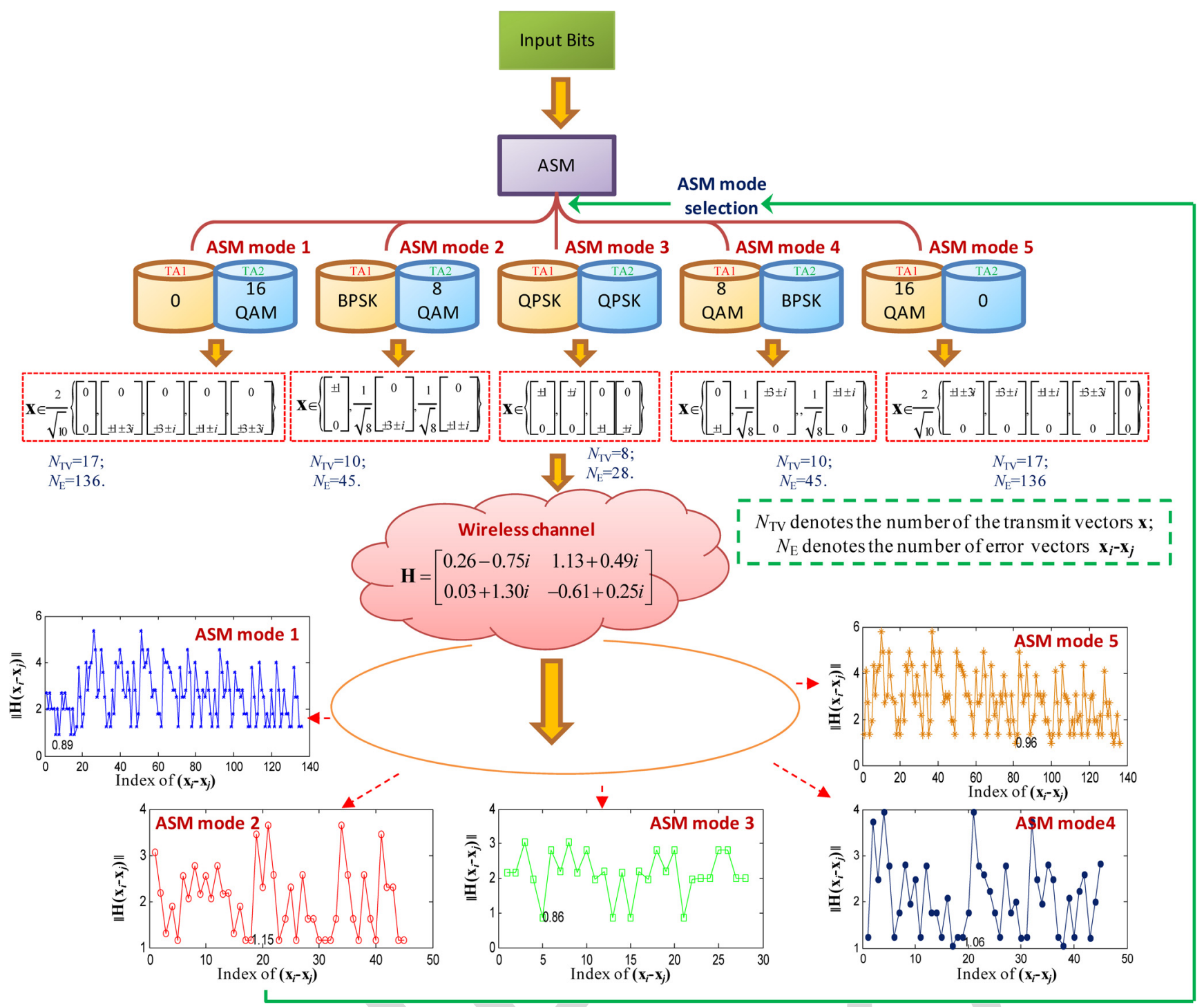

Fig. 10. The example of ASM associated with $(2 \times 2)$-element MIMO channels at a throughput of $m_{\text {all }}=3$ bits/symbol.

799 order combinations) are generated first. For each ASM mode, 800 we can calculate its legitimate transmit symbols $\mathrm{x}$ and its 801 corresponding error vectors. For example, as shown in Fig. 10, 802 the number of $\mathrm{x}$ combinations is $N_{\mathrm{TV}}=8$ for the ASM mode 3 803 (the candidate $[4,4]$ ), while the corresponding number of the 804 error vectors $\mathbf{e}_{i j}=\mathbf{x}_{i}-\mathbf{x}_{j}, i \neq j$ of (4) is $N_{E}=\left(\begin{array}{c}2 \\ N_{\mathrm{TV}}\end{array}\right)=28$. 805 Here, each error vector $\mathbf{e}_{i j}$ is given a specific index, which 806 is associated with its corresponding distance $\left\|\mathbf{H e}_{i j}\right\|$. Then, 807 the minimum value of $\left\|\mathbf{H e}_{i j}\right\|$ among all the legitimate error 808 vectors is found, which determines the FD of this ASM mode. 809 In Fig. 10, the FD of the ASM mode 3 is 0.86. For other ASM 810 modes, we can use the same method of determining the corre811 sponding FDs. Observe in Fig. 10 that ASM mode 2 has the 812 highest FD for the ASM candidate of [2,8]. The corresponding 813 ASM mode index 2 is then fed back to the transmitter.

814 As indicated above, the Modulation Order Selection (MOS) 815 of ASM turns out to be a demanding process, because the 816 global optimum is found by carrying out an exhaustive search 817 across the entire ASM's mode-candidate set. For example, for 818 an ASM scheme associated with $N_{\mathrm{t}}=8$ and 4 bits/symbol 819 transmission, we need a global search of 154,645 candidates, which results in an excessive complexity and feedback load, 820 when high data rates are required. To circumvent this problem, 821 the probabilities of occurrence for the ASM candidates were 822 evaluated theoretically in [119]. More specifically, all legiti- 823 mate ASM-mode candidates were classified according to their 824 variances and FD. It was shown that for most of the practical 825 channel realizations the probability that the maximum FD oc- 826 curs when all the TAs have the same modulation order is high. 827 As a result, only the specific ASM mode candidates associated 828 with lower variances were earmarked for the optimization in 829 Algorithm 3. Based on this result, a One Bit Re-Allocation 830 (OBRA) algorithm was proposed in [119] for the ASM mode 831 selection. OBRA-ASM imposes both a lower complexity and 832 a lower feedback requirement than that of the ASM relying 833 on a potentially excessive-complexity exhaustive search, while 834 imposing a marginal performance degradation. ${ }^{1}$

\footnotetext{
${ }^{1}$ Note that ASM may transmit an unequal number of bits in different time slot. Hence, this mismatch in the transmission frame-length will result in a potential error propagation effect at the detector, which may be mitigated using channel coding techniques, as detailed in [69].
} 


\section{B. Transmit Precoding Techniques}

837 Similar to the AM technique, Transmit Precoding (TPC) is 838 another attractive LA regime, which exploits the knowledge of 839 the CSI at the transmitter, in order to match the transmission 840 parameters to the instantaneous channel conditions. A bene841 ficial solution to this problem is to use the TPC matrix $\mathbf{P}$ 842 of (4) for enhancing the attainable performance. There is a 843 paucity of literature on how to design both linear and non-linear 844 precoders for conventional MIMO schemes [39]. To be specific, 845 non-linear precoding may be more powerful than its linear 846 counterparts, but linear TPC usually achieves a reasonable per847 formance at a significantly lower complexity. Moreover, most 848 of the precoders were designed using a capacity-maximization 849 approach [39], although in practice minimizing the BER may 850 be more important, than maximizing the mutual information or 851 the capacity [40].

852 1) Diagonal Precoding: The SM technique employed in 853 conjunction with a precoding scheme, where the transmitted 854 symbols are appropriately weighted according to the near855 instantaneous channel condition constitutes an attractive so856 lution in terms of improving the system's BER performance. 857 One of the key design challenges of the precoded SM-MIMO 858 architectures is to construct a beneficial precoding matrix $\mathbf{P}$ 859 that relies on a modest amount of feedback information, while 860 retaining all the single-RF benefits of SM-MIMOs.

861 To this end, in [103] a beamforming codebook was designed 862 for optimizing the coding gain of SM-MIMO in the presence 863 of spatial correlation amongst the fading envelopes of the TAs. 864 Recently, a closed-loop TPC method was invoked for providing 865 both diversity and coding gains in the context of GSSK [124], 866 which activated more than one TAs for transmission. However, 867 the above-mentioned schemes considered only a special case 868 of SM, namely SSK. As a result, the schemes proposed for 869 SSK may not be directly applicable to the conventional SM 870 scheme. By contrast, in [133] a TPC technique was used for 871 improving the signal design for a new class of SM, namely 872 for Receiver-SM (R-SM). Moreover, in [100] the authors in873 vestigated the effects of finite-alphabet inputs on the achievable 874 capacity of SM for transmission over MISO channels and 875 then developed a TPC scheme for improving this performance 876 metric.

877 In this section, we continue by considering a novel TPC 878 scheme based on maximizing the FD for the family of SM879 MIMO systems. Note that since the attainable performance of 880 the optimum single-stream ML receiver depends on the FD 881 of the received signal constellation [29], the maximization of 882 the FD directly reduces the probability of error. In order to 883 retain all the single-RF related benefits of SM, we designed 884 the TPC matrix $\mathbf{P}$ to be a diagonal matrix formulated as $885 \mathbf{P}=\operatorname{diag}\left\{p_{1}, \cdots, p_{n}, \cdots, p_{N_{t}}\right\}$. Note that although there are 886 various diagonal matrix aided TPCs proposed for the family 887 of conventional MIMO schemes, they tends to aim for diag888 onalizing the channel matrix [39], which may jeopardize the 889 advantages of SM-MIMOs. As a result, the conventional TPC 890 techniques proposed for classic MIMO schemes, such as the 891 STBC and VBLAST, may not be directly suitable for the family 892 of SM-MIMOs.
In order to identify the specific TPC parameters $p_{n}(n=893$ $1, \cdots, N_{t}$ ), which are capable of maximizing the FD, we have 894 to determine all the $N_{t}$ parameters $p_{n}\left(n=1, \cdots, N_{t}\right)$. Since 895 it may become excessively complex to jointly optimize these 896 $N_{t}$ parameters in the complex-valued field, we decomposed $\mathbf{P} 897$ as $\mathbf{P}=\overline{\mathbf{P}} \boldsymbol{\Theta}=\operatorname{diag}\left\{\bar{p}_{1} e^{j \theta_{1}}, \cdots, \bar{p}_{n} e^{j \theta_{n}}, \cdots, \bar{p}_{N_{t}} e^{j \theta_{N_{t}}}\right\}$. Be- 898 cause the FD of this particular TA-pair predominantly deter- 899 mines the achievable performance, only the specific TA pair 900 $(g, k)$ associated with the FD is considered and the TPC param- 901 eters are selected for appropriately weighting the SM symbols. 902 As a result, there are only two parameters, namely $p_{g}$ and $p_{k}, 903$ to be searched for. Finding the optimal values of $p_{g}$ and $p_{k}$ as 904 a function of both $\mathbf{H}$ and of the optimal transmit parameters 905 involves an exhaustive search over the vast design-space of 906 $\bar{p}_{g}, \bar{p}_{k}, \theta_{g}$ and $\theta_{k}$, which is overly complex. By considering 907 the power constraint, we have $\bar{p}_{k}=\sqrt{2-\bar{p}_{g}^{2}}$. Moreover, since 908 the phase rotation of the symbol is only carried by two TAs, 909 we can simplify the computation by fixing $\theta_{k}=0$ and then 910 finding the optimal $\theta_{g}$. The proposed low-complexity TPC 911 design algorithm is summarized as follows. 912

Algorithm 4: A low-complexity TPC design algorithm for 913 SM-MIMO

914

1) Given the transmit parameters $N_{t}, N_{r}$ and the transmis- 915 sion rate $m_{\text {all }}$ as well as the channel matrix $\mathbf{H}$, the indices 916 of the TA pair $(g, k)$ associated with the FD of (4) are 917 first obtained. In order to offer an increased FD, the TPC 918 parameters of this TA pair can be dynamically adapted. ${ }^{2} 919$

2) Generate all the legitimate diagonal TPC matrix candi- 920 dates represented as $\mathbf{P}_{\text {cand }}=\operatorname{diag}\left\{1, \cdots, \bar{p}_{g} e^{j \theta_{g}}, \cdots, 921\right.$ $\left.\sqrt{2-\bar{p}_{g}^{2}}, \cdots, 1\right\}$, where we have $\bar{p}_{g}=\sqrt{2} / L_{1} * l_{1}, l_{1}=922$ $0, \cdots, L_{1}$ and $\theta_{g}=2 \pi / L_{2} * l_{2}, l_{2}=0, \cdots, L_{2}$. Here, 923 $L_{1}$ and $L_{2}$ are the quantized parameters, which can 924 be flexibly selected according to the prevalent BER 925 requirements.

3) Based on the above-mentioned optimization rule, we 927 can achieve a performance gain by maximizing the FD 928 $d_{\min }(\mathbf{H})$ by switching among these TPC candidates. Note 929 that the FD of the TPC matrixes $\mathbf{P}_{\text {cand }}$ generated will be 930 compared to that of the conventional scheme and then we 931 select the one having the largest FD as our final result. 932

4) Then, the index of the optimized TPC matrix has to be 933 fed back to the transmitter.

934

Unlike in the traditional TPC method of [39], our proposed 935 scheme is suitable for scenarios relying bandwidth-limited 936 feedback channels, because the TPC design is reduced to the 937 design of a diagonal matrix. Moreover, as demonstrated in 938 Algorithm 4 as few as two elements of the diagonal TPC matrix 939 have to be fed back to the transmitter, regardless of the value 940 of $N_{t}$.

\footnotetext{
${ }^{2}$ Note that if the value of $g$ is the same as $k$, we have to adapt the TPC parameters of the pair $(g, u)$, where the TA $u$ has the maximum channel gain $\left\|\mathbf{h}_{u}\right\|_{F}$. Here, $\mathbf{h}_{u}$ is the uth column of $\mathbf{H}$ and $|\cdot|$ stands for the Frobenius norm.
} 


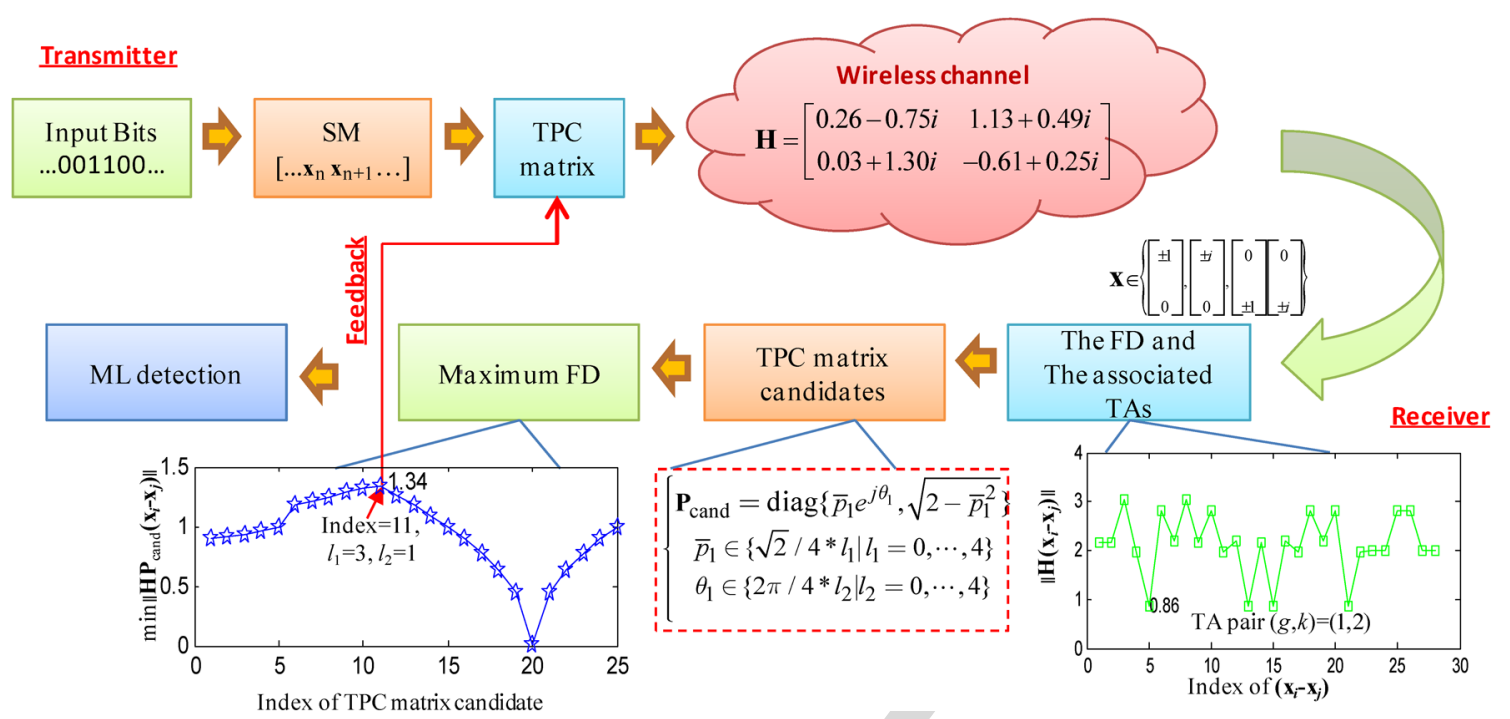

Fig. 11. The example of TPC aided SM.

942 More specifically, revisiting the previous example in 943 Algorithm 3, as shown in Fig. 11, for the same channel 944 realization $\mathbf{H}$, if the TPC matrix $\mathbf{P}$ of Algorithm 4 is used 945 for optimizing the system's performance, where the specific 946 TA-pair $(1,2)$ associated with the FD of 0.86 is first found by 947 using (4), which corresponds to the conventional SM scheme 948 (the ASM mode 3 in Fig. 10). This result implies that the FD 949 is computed for different TAs and the FD of this particular 950 TA-pair predominantly determines the achievable performance. 951 To improve the system's performance, the TPC parameters of 952 this pair should be optimized. Here, the optimized TPC matrix 953 is selected from the quantized TPC matrix set, as shown in 954 Fig. 11, where the quantized parameters $L_{1}$ and $L_{2}$ are se955 lected as $L_{1}=L_{2}=4$. Hence, the number of TPC candidates 956 is $\left(L_{1}+1\right) \times\left(L_{2}+1\right)=25$. We can assign a specific index 957 for each candidate and then calculate its corresponding FD 958 according to (4). As shown in Fig. 11, the specific candidate 959 associated with $l_{1}=3$ and $l_{2}=1$ has the highest FD of 1.34 960 among all the legitimate TPC matrix candidates. Note that if 961 the highest FD of all the legitimate TPC matrix candidates 962 is lower than that of the conventional SM. Based on step 3) 963 of Algorithm 4, The optimal TPC matrix is $\mathbf{P}=\mathbf{I}_{N_{t}}$. The 964 corresponding index of this candidate is then fed back to the 965 transmitter, which appropriately weights the SM modulated 966 symbol.

967 2) Phase Rotation Precoding and Power Allocation: Since 968 the proposed precoder $\mathbf{P}$ consists of two different diagonal 969 matrices $\overline{\mathbf{P}}$ and $\boldsymbol{\Theta}$, we may reduce the complexity of the 970 precoding process in Algorithm $\mathbf{4}$ by employing only a subset 971 of matrices at a modest performance loss. Firstly, when only the 972 diagonal matrix $\Theta$ is considered, this solution may be referred 973 to as the Phase Rotation Precoding (PRP) technique [134], 974 which is usually used for improving the BER, when spatial 975 correlation exists between the TAs of the ML-detection aided 976 V-BLAST architecture.

977 An alternative complexity reduction is achieved by consid978 ering only the diagonal matrix $\overline{\mathbf{P}}$, which can be viewed as 979 a simple form of Power Allocation (PA) [30], [121]-[123].
This arrangement has been intensively researched in the con- 980 text of spatial multiplexing systems [30]. However, these PA 981 approaches designed for spatial multiplexing based MIMO 982 systems may not be directly suitable for the family of SM- 983 MIMO systems, because only a single TA is active in each time 984 slot and hence the PA between the TAs should be carefully con- 985 sidered. In [30], an opportunistic power allocation scheme was 986 conceived for achieving a beneficial transmit diversity gain in 987 SSK-aided MIMO systems relying on two TAs. Then, this 988 feedback-aided PA scheme was further developed in [121]. 989 However, no APM scheme was considered in the above- 990 mentioned PA-aided SSK-MIMO systems and hence their 991 throughput may remain limited. In order to realize the full po- 992 tential of PA techniques in a SM-MIMO context, Algorithm 4993 can also be invoked by simply changing the legitimate diagonal 994 TPC matrix to the PA matrix.

Still considering the example given in Fig. 11, if the PA 996 technique is considered, we gradually assign the appropriate 997 portion of power to each TA of the TA pair $(1,2)$, where the 998 number of PA matrix candidates is $L_{1}+1=5$, as shown in 999 Fig. 12(a). Similar to Fig. 11, we can also assign a specific 1000 index for each candidate and then calculate its corresponding 1001 FD according to (4). As shown in Fig. 12(a), the PA matrix 1002 candidate associated with $l_{1}=3$ has the highest FD of 1.261003 among all the legitimate PA matrix candidates. On the other 1004 hand, as shown in Fig. 12(b), if the PRP technique is invoked, 1005 only the phases of the TA pair $(1,2)$ are adjusted, where the 1006 number of PRP matrix candidates is $L_{2}+1=5$. We observe 1007 from the results of Fig. 12(b) that the PRP matrix candidate 1008 associated with $l_{2}=3$ has the highest FD of 1.3 among all the 1009 legitimate PRP matrix candidates. The index of the optimized 1010 matrix is fed back to the transmitter for allowing the transmitter 1011 to compensate for the effects of channel fading.

1012

3) Performance Results: In Fig. 13, we compared the var- 1013 ious LA-aided SM schemes to the conventional non-adaptive 1014 SM scheme in the context of $(2 \times 2)$-element MIMO chan- 1015 nels at a throughput of $m_{\text {all }}=3$ bits/symbol for transmission 1016 over independent Rayleigh block-flat channels. In all cases we 1017 


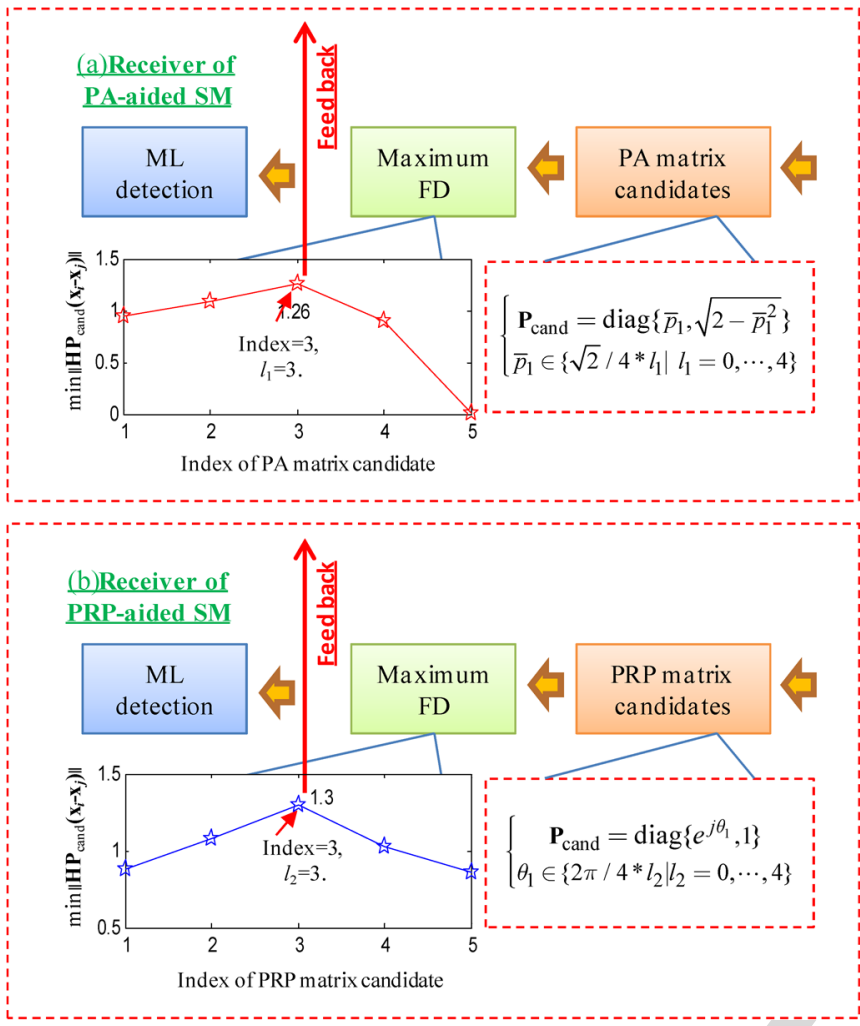

Fig. 12. The example of PA and PRP aided SM system.

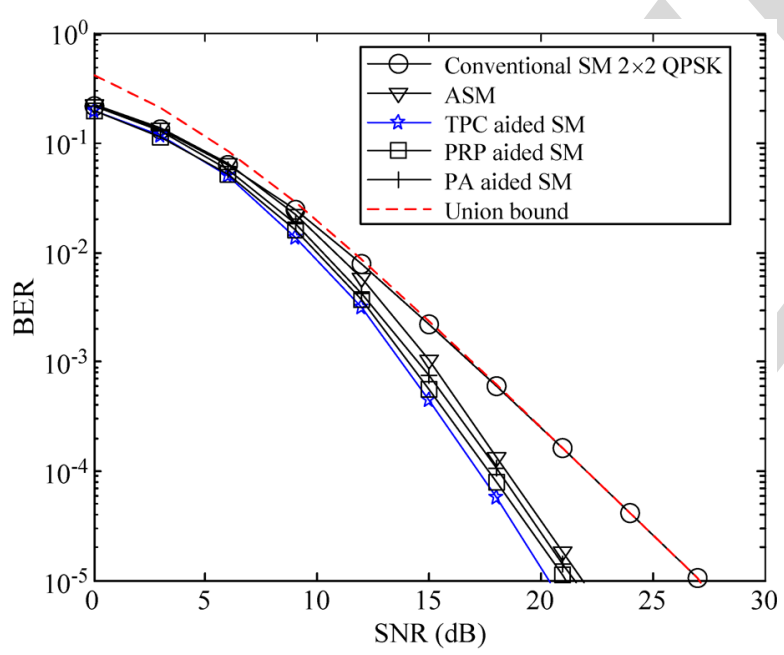

Fig. 13. BER performance of the conventional SM and the LA-aided SM schemes in $(2 \times 2)$-element MIMO channels at a throughput of $m_{\text {all }}=$ 3 bits/symbol.

1018 assumed that the feedback channel is free of errors and delay. ${ }^{3}$ 1019 For completeness, we also added the theoretical upper bound 1020 curve derived with the aid of the union bound [29], [103] of 1021 the conventional SM scheme. Moreover, in the TPC design of 1022 Algorithm 4, we selected $L_{1}=L_{2}=4$.

\footnotetext{
${ }^{3}$ The error-free feedback channel assumption in SM-based schemes may be justifiable, since the feedback channel is usually protected using powerful error correction coding and hence has a low error probability [4]. The effect of imperfect feedback channels in closed-loop MIMO systems has been documented, for example in [135].
}

As expected, the proposed LA-aided schemes beneficially 1023 exploit the flexibility of the transmit parameters and as seen 1024 in Fig. 13, they provide an SNR gain of about 5.1-7.3 dB over 1025 the conventional SM scheme at the BER of $10^{-5}$. Moreover, the 1026 TPC-aided SM achieves the best BER performance amongst all 1027 benchmark schemes, as seen in Fig. 13. This is mainly due to 1028 the fact that the PA-assisted SM and PRP-aided SM schemes 1029 are simplified versions of the TPC-aided SM scheme, which 1030 have a suboptimal BER performance. Moreover, the selection 1031 of TPC parameters is more flexible than that of ASM, because 1032 the modulation orders of ASM are selected from a discrete set, 1033 while the TPC parameters are chosen from the vast complex- 1034 valued field. The performance gain of the TPC-aided SM over 1035 ASM is explicitly seen in Fig. 13.

\section{Antenna Selection}

Antenna Selection (AS) constitutes another promising low- 1038 cost technique, since it enjoys the full-diversity benefits offered 1039 by MIMO architectures at the cost of requiring a low feedback 1040 rate. Due to its advantages, AS has been adopted in contempo- 1041 rary wireless systems such as IEEE 802.11n [136]. A detailed 1042 overview of AS techniques was presented in [136] and both 1043 the so-called norm-based selection and the successive selection 1044 scheme were detailed. Recently, a systematic overview of all 1045 physical and higher layer features of the LTE standard relying 1046 on Transmit AS (TAS) were presented in [137]. To be specific, 1047 TAS has been adopted by LTE for both its Frequency Division 1048 Duplexing (FDD) and Time Division Duplexing (TDD) modes 1049 of operation.

SM can also be beneficially combined with the AS technique 1051 for the sake of enhancing its performance. In recent years, 1052 several AS methods have been introduced and extended to the 1053 class of SM-MIMO systems with the goal of enhancing its 1054 capacity or its BER. For example, in [127], a TAS method based 1055 on exhaustive search was proposed for exploiting the available 1056 CSI. As natural extensions of the existing literature on TAS 1057 for spatial multiplexing systems, in [128], a low-complexity 1058 maximum-ED based TAS method and a maximum-capacity 1059 TAS method were investigated. Moreover, three closed-loop 1060 AS-aided SSK schemes were proposed in [126], which relied 1061 on the classic norm-based AS criterion, on the minimal PEP 1062 criterion and on their hybrid.

\section{Hybrid Adaptation and Other LA Schemes}

As mentioned in Section IV-A, ASM is capable of transmit- 1065 ting different number of bits over different TAs. Hence this 1066 scheme may achieve increased benefits due to the associated 1067 channel gain difference by exploiting it with the aid of dis- 1068 similar channel matrix column vectors [104]. For example, 1069 as shown in Algorithm 3, the number of bits carried by the 1070 conventional 4-QAM symbol is 2 in each SM symbol, while 1071 the number of bits conveyed by the TA indices is only one. 1072 The AM scheme is capable of varying this bit-mapping strategy 1073 according to the near-instantaneous channel conditions, while 1074 the TPC aided schemes [30], [103], [121]-[125] have to utilize 1075 a fixed modulation order and hence they may fail to achieve 1076 
1077 this level of flexibility. However, TPC exhibits an extra grade 1078 of flexibility, since it can have arbitrary coefficients.

1079 As discussed in the context of (4) and Fig. 9, apart from 1080 adapting the APM modes, LA-aided SM can also benefit from 1081 adapting the TPC parameters for the sake of improving the sys1082 tem's performance. For example, when a high power amplifier 1083 efficiency and a high transmission rate are required, the classic 1084 PSK scheme may be preferred to QAM in diverse SM-MIMO 1085 configurations both in terms of its BER and PAPR, because 1086 PSK may be conveniently combined with the above-mentioned 1087 PRP technique for creating a PRP-aided constant-modulus SM 1088 scheme. In this scheme, the APM constellation optimization 1089 technique of Section III may be efficiently combined with 1090 the TPC technique of Section IV-B for improving both the 1091 achievable energy efficiency and the BER performance.

\section{V. FURTHER SM-RELATED STUdiES}

\section{A. Cooperative SM-Related Systems}

1094 Cooperative techniques are capable of gleaning some of 1095 the advantages of classic multiple-antenna aided transmission 1096 techniques with the aid of cooperating single-antenna assisted 1097 nodes within a network [139]. Based on a philosophy similar to 1098 that of the STC-based schemes, relay-aided SM schemes have 1099 been proposed in [140]-[147]. For example, in [140], a decode1100 and-forward (DF) relaying aided coherent STSK system was 1101 proposed, where the dispersion-vector was activated based on 1102 cyclic redundancy checking (CRC)-assisted error detection. 1103 The proposed design is capable of adapting both the number of 1104 the RNs as well as the transmission rate and the achievable di1105 versity order, depending on the associated system requirements 1106 and channel conditions. Moreover, a differentially-encoded and 1107 non-coherently detected version of STSK was developed in 1108 [140], which dispenses with CSI estimation at all of the nodes, 1109 while retaining the benefits of the cooperative coherent STSK. 1110 In order to further improve the cooperative STSK's perfor1111 mance as well as to combat the effects of frequency-selective 1112 channels, in [141], Successive-Relaying (SR)aided cooperative 1113 multicarrier (MC) STSK was proposed. This technique invokes 1114 the selective DF and SR principles for the sake of recovering the 1115 half-duplex multiplexing loss while relying on the MC Code1116 Division Multiple Access (MC-CDMA) [148] principle for 1117 supporting multiple users, and simultaneously circumventing 1118 the dispersive effects of wireless channels. Moreover, in [142] 1119 a so-called Information Guided Transmission (IGT) scheme 1120 was employed for carrying out the random selection of the 1121 active nodes from the set of candidate Relay Nodes (RNs) for 1122 the sake of achieving a high relay throughput. Note that the 1123 above-mentioned SM-related cooperative systems may rely on 1124 single-antenna based transmissions at the Source-Node (SN), 1125 but some form of loose inter-relay synchronization (IRS) should 1126 be considered, unless the so-called Large-Area-Synchronized 1127 (LAS) spreading codes of [149], [150] are employed.

1128 Moreover, in [121], an Amplify-and-Forward (AF)-relaying1129 aided SSK scheme was conceived for reducing the number 1130 of TAs and for mitigating the effects of deep fading. More 1131 recently, Mesleh et al. [143], [144] invoked dual-hop AF and
DF relaying aided SSK schemes, which were characterized 1132 by the corresponding BER performance upper-bounds. How- 1133 ever, as mentioned in Section II, the throughput of the SSK- 1134 aided cooperative schemes may remain somewhat limited. To 1135 eliminate this impediment, a dual-hop cooperative SM scheme 1136 [145] was conceived for combining SSK with classic APM 1137 techniques for the sake of transmitting additional bits. More 1138 specifically, the spatial domain of dual-hop SM has been ex- 1139 ploited for transmitting additional information bits, hence this 1140 system may have the potential of providing substantial spectral 1141 efficiency and coding gains in the context of wireless relay 1142 networks. In [146], the SSK-MIMO principle is studied for 1143 the uplink of cellular networks. The source broadcasts its data 1144 packet to the available relays. The data packets are decoded by 1145 each relay individually and each decoded symbol is compared 1146 against unique identifiers of the relays. The specific relays 1147 that demodulate the data associated with their own identifier 1148 become active and transmit the associated SSK symbol to the 1149 destination. Hence, the set of relays act as a distributed spatial- 1150 constellation diagram for the source, similar to the SSK-MIMO 1151 communications concept with co-located TAs. The distributed 1152 encoding principle of [146] was then extended in [147] with 1153 the objective of improving the achievable bandwidth efficiency 1154 of half-duplex relaying. The associated transmission protocol 1155 is similar to that of [146], with one main exception, namely 1156 that active relays transmit the first data packet stored in their 1157 buffers during the second phase. This enables the relay to 1158 simultaneously transmit both the data received from the source 1159 and its own data. This is due to the fact that when a relay 1160 is active, the source data is conveyed by conventional APM 1161 modulation through this relay, while an additional data symbol 1162 can be implicitly mapped onto the relay's index. The results 1163 show that the adoption of a distributed SM-MIMO scheme is 1164 indeed capable of improving the attainable performance.

\section{B. SM-Related Systems for Frequency Selective Channels}

Despite its rich literature, the family of SM-related schemes 1167 has been predominantly investigated in the context of single- 1168 user flat fading channels. However, in high-rate SM-MIMO 1169 communication systems, the Inter-Symbol Interference (ISI) 1170 caused by multipath components of the frequency selective 1171 channel has to be considered.

Hence various SM-related systems have been investigated 1173 not only in the context of single carrier (SC) contexts [148] 1174 and but also in multi-carrier systems [151]. More specifically, 1175 in [55] the authors proposed the STFSK regime for overcoming 1176 the effects of dispersive channels, while striking a flexible 1177 trade-off between the attainable diversity and multiplexing 1178 gain. STFSK is capable of flexibly exploiting the available 1179 time-, space- and frequency-diversity, hence attaining an attrac- 1180 tive performance in frequency-selective fading environments. 1181 In [152], an OFDM-aided STSK system was proposed, which 1182 achieves almost the same BER performance as that of its single- 1183 carrier counterpart operating in a narrowband channel. More- 1184 over, in order to support high-rate multiuser transmissions, a 1185 novel multiuser STSK scheme was conceived for frequency- 1186 selective channels in [153], which was combined with the 1187 
1188 classic OFDMA/SC-FDMA techniques for the sake of con1189 verting the frequency-selective wideband channel to numerous 1190 parallel non-dispersive narrowband channels. In [154], an 1191 antenna-hopping space-division multiple-access aided SM 1192 scheme was advocated for exploiting the advantages of SM. 1193 For efficiently detecting this scheme, a range of linear and non1194 linear detection schemes have been investigated.

1195 Recently, SM-related schemes were investigated in a fre1196 quency selective channel by combining the classic Cyclic1197 Prefixed (CP) single carrier technique [155], [156], which is 1198 capable of avoiding the PAPR problem encountered in mul1199 ticarrier based systems. A comparison between CP-aided SC$1200 \mathrm{SM}$ and the CP-aided SM-OFDM systems was also presented 1201 for the sake of identifying the advantages of the single-carrier1202 SM scheme. Then, Rajashekar et al. further generalized the 1203 solutions of [156], where a Zero-Padded (ZP) single-carrier 1204 SM system was proposed for achieving the maximum attain1205 able multi-path diversity order with the aid of low-complexity 1206 single-stream ML detection. It was shown that the proposed ZP1207 aided SC-SM system provides beneficial system performance 1208 improvements compared to both the CP-aided SC-SM and the 1209 CP-aided SM-OFDM systems.

\section{C. The Energy-Efficient SM-Related Systems}

1211 Recently, the energy consumption issue in wireless commu1212 nication has attracted increasing attention, especially in MIMO1213 aided LTE and LTE-A networks [111], [157]. As a new kind of 1214 MIMO transmission technique and a promising candidate for 1215 future wireless applications and standards, SM can be realized 1216 by using a single RF front-end, hence it has a high power1217 efficiency [15]. However, how to further improve the energy1218 efficiency of SM-MIMO schemes is important in practical 1219 deployments.

1220 Some of the above-mentioned issues have been already 1221 investigated in [16], [17], [69], and [158]. More specifi1222 cally, in [16] the authors evaluated the energy efficiency of a 1223 multi-antenna assisted base station employing SM based on a 1224 realistic power consumption model. It was found that the SM1225 aided base station has a considerable power consumption gain 1226 compared to multi-RF chain aided MIMO arrangements. This 1227 advantage of SM was further confirmed in [17] by considering 1228 different base station types. Then, in [158], the energy con1229 sumption of a class of adaptive SM was evaluated. Moreover, 1230 in [69], an energy-efficient SM-MIMO scheme was designed, 1231 which relied on the Hamming coding and Huffman coding 1232 techniques. This scheme was capable of striking a flexible 1233 spectral-efficiency versus energy-efficiency tradeoff. Note that 1234 although the above-mentioned research demonstrated that SM 1235 constitutes an energy-efficient design [111], [157], the current 1236 research results are still preliminary and hence further investi1237 gations are required.

\section{CONCLUSION}

\section{A. Summary of the Paper}

1240 In this tutorial, we reviewed a range of recent research 1241 achievements on SM and its potential applications. We consid- ered some of its transceiver design aspects, the spatial constel- 1242 lation optimization, the associated link adaptation techniques, 1243 the distributed/cooperative system design issues and their ben- 1244 eficial combinations.

In Section II, we provided a rudimentary system overview 1246 of the conventional SM technique and its variants, empha- 1247 sizing the associated transceiver design techniques for strik- 1248 ing an attractive trade-off amongst the range of potentially 1249 conflicting system requirements. More specifically, the bit-to- 1250 symbol mapping principle of the SM transmitter was presented 1251 Section II-A. Then, various generalized versions of SM were 1252 introduced in Section II-B. Section II-C summarized the class 1253 of hard- and soft-detection techniques designed for SM-related 1254 schemes, which was roughly divided into four fundamental 1255 categories. In Section II-D, both the channel capacity and error 1256 performance metrics of SM-related schemes were summarized, 1257 which were used as a reference for the sake of highlighting 1258 the advantages of SM compared to other MIMO arrangements. 1259 These metrics were also used for system optimization by ex- 1260 ploiting the knowledge of CSI.

In Section III, the effects of APM schemes on the perfor- 1262 mance of SM were characterized and we proposed a class of 1263 star-QAM constellations for minimizing the system's BER. In 1264 Section IV, we introduced the family of limited-feedback aided 1265 LA techniques designed for SM-related schemes. Depending on 1266 the specific degree of freedom exploited, these techniques were 1267 divided into four types constituted by AM, TPC, AS and their 1268 hybrid techniques. Specifically, the near-instantaneously ASM 1269 scheme of Section IV-A has been proposed in [104], [115], 1270 and [119] for improving the attainable system performance, 1271 while maintaining a fixed average transmit rate with the aid 1272 of AM techniques. Moreover, the diagonal TPC scheme of 1273 Section IV-B has been proposed in [118] and [122] based 1274 on maximizing the FD for the family of SM-MIMO systems, 1275 where the transmitted symbols are appropriately pre-weighted 1276 according to the channel condition. Finally, we discussed a 1277 variety of other SM-related classes including those designed for 1278 frequency selective channels, for cooperative SM scenarios and 1279 for energy-efficient applications.

\section{B. Future Research Ideas}

In this paper, we considered only the minimum-distance 1282 based approach of extracting the LA parameters, in order to 1283 achieve beneficial performance improvements in the high-SNR 1284 regime. As further work, one can formulate and solve the LA 1285 problems by considering a range of other optimization criteria 1286 depending on the amount of channel state information available 1287 as well as on other system requirements, such as capacity- and 1288 SNR-optimized design rules [39]. Moreover, the integration of 1289 trellis coding as well as space-time block coding and other 1290 coding techniques [4] into the proposed LA schemes may also 1291 be further researched. Perhaps the most challenging of all is 1292 the design of non-coherent detection aided or blind-detection 1293 assisted schemes, which are capable of dispensing with channel 1294 information. These are particularly important in the context of 1295 relay-aided systems, where the source-relay channel cannot be 1296 readily estimated. 
1298

\section{ABEP}

1300 AM

1301 APM

1302 AS

1303 ASM

1304 BER

1305 BP-IGCH

$1306 \mathrm{CS}$

1307 CSI

1308 C-SM

1309 CSTSK

1310 DSTC

$1311 \mathrm{ED}$

$1312 \mathrm{EMF}$

1313 FD

1314 FSK

1315 FBE

1316 GSM

1317 G-STSK

1318 IAI

1319 IAS

$1320 \mathrm{IGCH}$

1321 ISI

1322 LA

1323 LTE

1324 MA-SM

1325 MAP

$1326 \mathrm{MF}$

1327 MIMO

1328 MISO

$1329 \mathrm{ML}$

1330 MMD

1331 MOS

1332 MRC

$1333 \mathrm{NMF}$

1334 OFDM

1335 OFDMA

1336 OFDM-IM

$1337 \mathrm{OH}-\mathrm{SM}$

1338 OSD

1339 OSDM

1340 PA

1341 PAPR

1342 PEP

1343 PRP

1344 PSK

1345 QAM

$1346 \mathrm{RF}$

1347 R-SM

1348 SC-FDMA

1349

$1350 \mathrm{SD}$

1351 SDM

1352 SFSK

1353 SIM

1354 SISO
VII. GLOSSARY

Average Bit Error Probability.

Adaptive Modulation.

Amplitude and Phase Modulation.

Antenna Selection.

Adaptive SM.

Bit Error Ratio.

Bit-Padding IGCH.

Compressed Sensing.

Channel State Information.

Concatenated SM.

Coherent STSK.

Differentially-encoded STC.

Euclidean distance.

Exhaustive-search MF.

Free Distance.

Frequency-Shift Keying.

Fractional Bit Encoded.

Generalized SM.

Generalized STSK.

Inter Antenna Interference.

Inter-Antenna-Synchronization.

Information-Guided Channel Hopping.

Inter-Symbol Interference.

Link adaptation.

Long-Term Evolution.

Multiple Active-SM.

Maximum a posteriori.

Matched Filter.

Multiple-Input Multiple-Output.

Multiple-Input Single-Output.

Maximum Likelihood.

Maximum-Minimum Distance.

Modulation Order Selection.

Maximum Ratio Combining.

Near-optimal MF.

Orthogonal Frequency-Division Multiplexing.

Orthogonal Frequency Division Multiple Access.

OFDM with Index Modulation.

Optimal Hybrid-SM.

Ordered SD.

Orthogonal Spatial-Division Multiplexing.

Power Allocation.

Peak-to-Average-Power Ratio.

Pairwise Error Probability.

Phase Rotation Precoding.

Phase Shift Keying.

Quadrature Amplitude Modulation.

Radio Frequency.

Receiver-SM.

Single-Carrier Frequency Division Multiple Access.

Sphere Decoding.

Spatial Division Multiplexing.

Space-Frequency Shift Keying.

Subcarrier-Index Modulation.

Single-Input Single-Output.
SM Spatial Modulation.

1355

STBC Space Time Block Codes. 1356

STC Space-Time Coding. 1357

STFSK Space-Time-Frequency Shift Keying. 1358

SSK $\quad$ Space Shift Keying. 1359

STSK Space-Time Shift Keying. $\quad 1360$

VBD $\quad$ Vector Based Detection. 1361

TA Transmit Antenna. 1362

TAS Transmit Antenna Selection. 1363

TC Trellis Coding. 1364

TCM Trellis Coded Modulation. 1365

TOSD-SM Time-Orthogonal Signal Design assisted SM. 1366

TPC Transmit Precoding. 1367

TMS Transmit Mode Switching. $\quad 1368$

USTM Unitary Space-Time Modulation. 1369

V-BLAST Vertical-Bell Laboratories Layered Space-Time. 1370
1371

[1] G. J. Foschini and M. J. Gans, "On limits of wireless communications 1372 in a fading environment when using multiple antennas," Wireless Pers. 1373 Commun., vol. 6, no. 3, pp. 311-335, Mar. 1998.

[2] I. E. Telatar, "Capacity of multi-antenna Gaussian channels," Eur. Trans. 1375 Telecommun., vol. 10, no. 6, pp. 585-595, Nov./Dec. 1999.

[3] V. Tarokh, N. Seshadri, and A. R. Calderbank, "Space-time codes for 1377 high data rate wireless communication: Performance criterion and code 1378 construction," IEEE Trans. Inf. Theory, vol. 44, no. 2, pp. 744-765, 1379 Mar. 1998.

1380

[4] L. Hanzo, O. Alamri, M. El-Hajjar, and N. Wu, Near-Capacity Multi- 1381 Functional MIMO Systems: Sphere-Packing, Iterative Detection, and 1382 Cooperation. Hoboken, NJ, USA: Wiley, 2009. 1383

[5] Q. Li et al., "MIMO techniques in WiMAX and LTE: A feature 1384 overview," IEEE Commun. Mag., vol. 48, no. 5, pp. 86-92, May 2010. 1385

[6] F. Boccardi et al., "Multiple-antenna techniques in LTE-advanced," 1386 IEEE Commun. Mag., vol. 50, no. 3, pp. 114-121, Mar. 2012.

[7] S. Sugiura, S. Chen, and L. Hanzo, "MIMO-aided near-capacity turbo 1388 transceivers: Taxonomy and performance versus complexity," IEEE 1389 Commun. Surveys Tuts., vol. 14, no. 2, pp. 421-442, Second Quarter 1390 2012.

1391

[8] S. Sugiura, S. Chen, and L. Hanzo, "A universal space-time architec- 1392 ture for multiple-antenna aided systems," IEEE Commun. Surveys Tuts., 1393 vol. 14, no. 2, pp. 401-420, Second Quarter 2012.

[9] J. Mietzner, R. Schober, L. Lampe, W. H. Gerstacker, and P. A. Hoeher, 1395 "Multiple-antenna techniques for wireless communications-A compre- 1396 hensive literature survey," IEEE Commun. Surveys Tuts., vol. 11, no. 2, 1397 pp. 87-105, Second Quarter 2009.

1398

[10] L. Zheng and D. N. C. Tse, "Diversity and multiplexing: A fundamental 1399 tradeoff in multiple-antenna channels," IEEE Trans. Inf. Theory, vol. 49, 1400 no. 5, pp. 1073-1096, May 2003.

1401

[11] R. Y. Mesleh, H. Haas, S. Sinanovic, C. W. Ahn, and S. Yun, "Spatial 1402 modulation," IEEE Trans. Veh. Technol., vol. 57, no. 4, pp. 2228-2241, 1403 Jul. 2008.

1404

[12] Y. Yang and B. Jiao, "Information-guided channel-hopping for high 1405 data rate wireless communication," IEEE Commun. Lett., vol. 12, no. 4, 1406 pp. 225-227, Apr. 2008.

1407

[13] L. Hanzo, S. X. Ng, T. Keller, and W. Webb, Quadrature Amplitude 1408 Modulation: From Basics to Adaptive Trellis-Coded, Turbo-Equalised 1409 and Space-Time Coded OFDM, CDMA and MC-CDMA Systems. 1410 Hoboken, NJ, USA: Wiley, 2004.

[14] M. Di Renzo, H. Haas, and P. M. Grant, "Spatial modulation for 1412 multiple-antenna wireless systems: A survey," IEEE Commun. Mag., 1413 vol. 49, no. 12, pp. 182-191, Dec. 2011.

[15] M. Di Renzo, H. Haas, A. Ghrayeb, S. Sugiura, and L. Hanzo, "Spatial 1415 modulation for generalized MIMO: Challenges, opportunities and im- 1416 plementation," Proc. IEEE, vol. 102, no. 1, pp. 56-103, Jan. 2014.

[16] A. Stavridis, S. Sinanovic, M. Di Renzo, H. Haas, and P. M. Grant, "An 1418 energy saving base station employing spatial modulation," in Proc. IEEE 1419 Int. Workshop Comput.-Aided Model. Anal. Des. Commun. Links Netw., 1420 Barcelona, Spain, Sep. 2012, pp. 231-235.

[17] A. Stavridis, S. Sinanovic, M. Di Renzo, and H. Haas, "Energy 1422 evaluation of spatial modulation at a multi-antenna base station," in 1423 
Proc. IEEE Veh. Technol. Conf.-Fall, Barcelona, Spain, Sep. 2013, pp. 1-5.

[18] T. L. Marzetta, "Noncooperative cellular wireless with unlimited numbers of base station antennas," IEEE Trans. Wireless Commun., vol. 9, no. 11, pp. 3590-3600, Nov. 2010.

[19] F. Rusek et al., "Scaling up MIMO: Opportunities and challenges with very large arrays," IEEE Signal Proces. Mag., vol. 30, no. 1, pp. 40-46, Jan. 2013.

[20] R. Mesleh, H. Elgala, and H. Haas, "Optical spatial modulation," IEEE/OSA J. Optical Commun. Netw., vol. 3, no. 3, pp. 234-244, Mar. 2011.

[21] W. Popoola, E. Poves, and H. Haas, "Error performance of generalised space shift keying for indoor visible light communications," IEEE Trans. Commun., vol. 61, no. 5, pp. 1968-1976, May 2013.

[22] W. Popoola, E. Poves, and H. Haas, "Spatial pulse position modulation for optical communications," EEE/OSA J. Lightw. Technol., vol. 30, no. 18, pp. 2948-2954, Sep. 2012.

23] C. Vladeanu, R. Lucaciu, and A. Mihaescu, "Optical spatial modulation for indoor wireless communications in presence of inter-symbol interference," in Proc. IEEE Int. Symp. Electron. Telecommun., Nov. 2012, pp. 183-186.

24] E. Poves, W. Popoola, H. Haas, J. Thompson, and D. Cardenas, "Experimental results on the performance of optical spatial modulation systems," in Proc. IEEE Veh. Technol. Conf._Fall, Quebec City, QC, Canada, Sep. 2012, pp. 1-5.

25] A. Younis et al., "Performance of spatial modulation using measured real-world channels," in IEEE Veh. Technol. Conf.-Fall, Las Vegas, NV, USA, 2013, pp. 1-5.

26] N. Serafimovski et al., "Practical implementation of spatial modulation," IEEE Trans. Veh. Technol., vol. 62, no. 9, pp. 4511-4523, Nov. 2013.

27] M. Di Renzo and H. Haas, "A general framework for performance analysis of space shift keying (SSK) modulation for MISO correlated Nakagami-m fading channels," IEEE Trans. Commun., vol. 58, no. 9, pp. 2590-2603, Sep. 2010

28] M. Di Renzo and H. Haas, "Space shift keying (SSK-) MIMO over correlated Rician fading channels: Performance analysis and a new method for transmit-diversity," IEEE Trans. Commun., vol. 59, no. 1, pp. 116129, Jan. 2011.

29] M. Di Renzo and H. Haas, "Bit error probability of SM-MIMO over generalized fading channels," IEEE Trans. Veh. Technol., vol. 61, no. 3, pp. 1124-1144, Mar. 2012

[30] M. Di Renzo and H. Haas, "Improving the performance of space shift keying (SSK) modulation via opportunistic power allocation," IEEE Commun. Lett., vol. 14, no. 6, pp. 500-502, Jun. 2010.

31] M. Di Renzo and H. Haas, "On transmit-diversity for spatial modulation MIMO: Impact of spatial-constellation diagram and shaping filters at the transmitter," IEEE Trans. Veh. Technol., vol. 62, no. 6, pp. 2507-2531, Jul. 2013.

[32] M. Di Renzo and H. Haas, "Bit error probability of space shift keying MIMO over multiple-access independent fading channels," IEEE Trans. Veh. Technol., vol. 60, no. 8, pp. 3694-3711, Oct. 2011.

[33] M. Di Renzo and H. Haas, "Space shift keying (SSK) modulation with partial channel state information: Optimal detector and performance analysis over fading channels," IEEE Trans. Commun., vol. 58, no. 11, pp. 3196-3210, Nov. 2010.

34] M. Di Renzo, D. De Leonardis, F. Graziosi, and H. Haas, "Space shift keying (SSK-) MIMO with practical channel estimates," IEEE Trans. Commun., vol. 60, no. 4, pp. 998-1012, Apr. 2012.

35] S. M. Alamouti, "A simple transmit diversity technique for wireless communications," IEEE J. Sel. Areas Commun., vol. 16, no. 8, pp. 14511458, Oct. 1998.

[36] P. W. Wolniansky, G. J. Foschini, G. D. Golden, and R. A. Valenzuela, "V-BLAST: An architecture for realizing very high data rates over the rich-scattering wireless channel," in Proc. ISSSE, Pisa, Italy, 1998, pp. $295-300$.

37] B. Hassibi and B. M. Hochwald, "High-rate codes that are linear in space and time," IEEE Trans. Inf. Theory, vol. 48, no. 7, pp. 1804-1824, Jul. 2002.

[38] L. Hanzo, J. Blogh, and S. Ni, 3G, HSPA and FDD Versus TDD Networking: Smart Antennas and Adaptive Modulation. Hoboken, NJ, USA: Wiley, 2008.

39] D. J. Love et al., "An overview of limited feedback in wireless communication systems," IEEE J. Sel. Areas Commun., vol. 26, no. 8, pp. 13411365, Oct. 2008.

[40] C. B. Chae, A. Forenza, R. W. Heath, Jr., M. R. Mckay, and I. B. Collings, "Adaptive MIMO transmission techniques for broadband wireless communication systems," IEEE Commun. Mag., vol. 48, no. 5, 1501 pp. 112-118, May 2010.

[41] S. Catreux, V. Erceg, D. Gesbert, and R. W. Heath, Jr., "Adaptive mod- 1503 ulation and MIMO coding for broadband wireless data networks," IEEE 1504 Commun. Mag., vol. 40, no. 6, pp. 108-115, Jun. 2002.

[42] H. Haas, E. Costa, and E. Schultz, "Increasing spectral efficiency by 1506 data multiplexing using antennas arrays," in Proc. IEEE Int. Symp. Pers., 1507 Indoor, Mobile Radio Commun., Sep. 2002, vol. 2, pp. 610-613. 1508

[43] S. Sugiura, S. Chen, and L. Hanzo, "Coherent and differential 1509 space-time shift keying: A dispersion matrix approach," IEEE Trans. 1510 Commun., vol. 58, no. 11, pp. 3219-3230, Nov. 2010.

[44] J. Jeganathan, A. Ghrayeb, L. Szczecinski, and A. Ceron, "Space 1512 shift keying modulation for MIMO channels," IEEE Trans. Wireless 1513 Commun., vol. 8, no. 7, pp. 3692-3703, Jul. 2009.

[45] Y. Chau and S.-H. Yu, "Space modulation on wireless fading chan- 1515 nels," in Proc. IEEE Veh. Technol. Conf._Fall, Atlantic City, NJ, USA, 1516 Oct. 2001, vol. 3, pp. 1668-1671.

[46] J. Jeganathan, A. Ghrayeb, and L. Szczecinski, "Generalized space shift 1518 keying modulation for MIMO channels," in Proc. IEEE Int. Symp. Pers., 1519 Indoor, Mobile Radio Commun., Cannes, France, Sep. 2008, pp. 1-5. 1520

[47] J. Fu, C. Hou, W. Xiang, L. Yan, and Y. Hou, "Generalised spatial modu- 1521 lation with multiple active transmit antennas," in Proc. IEEE Globecom 1522 Workshops, Miami, FL, USA, Dec. 2010, pp. 839-844.

[48] A. Younis, N. Serafimovski, R. Mesleh, and H. Haas, "Generalised 1524 spatial modulation," in Proc. Signals, Syst. Comput., Pacific Grove, CA, 1525 USA, Nov. 2010, pp. 1498-1502.

[49] J. Wang, S. Jia, and J. Song, "Generalised spatial modulation system 1527 with multiple active transmit antennas and low complexity detection 1528 scheme," IEEE Trans. Wireless Commun., vol. 11, no. 4, pp. 1605-1615, 1529 Apr. 2012.

[50] E. Başar, Ü. Aygölü, E. Panayırc1, and H. V. Poor, "Space-time block 1531 coded spatial modulation," IEEE Trans. Commun., vol. 59, no. 3, 1532 pp. 823-832, Mar. 2011.

[51] R. Y. Mesleh, M. Di Renzo, H. Haas, and P. M. Grant, "Trellis coded spa- 1534 tial modulation," IEEE Trans. Wireless Commun., vol. 9, no. 7, pp. 2349- 1535 2361, Jul. 2010.

[52] E. Başar, Ü. Aygölü, E. Panayırc1, and H. V. Poor, "New trellis code 1537 design for spatial modulation," IEEE Trans. Wireless Commun., vol. 10, 1538 no. 8, pp. 2670-2680, Aug. 2011.

[53] E. Başar, Ü. Aygölü, E. Panayırcı, and H. V. Poor, "Super-orthogonal 1540 trellis-coded spatial modulation," IET Commun., vol. 6, no. 17, 1541 pp. 2922-2932, Nov. 2012.

[54] S. Sugiura, S. Chen, and L. Hanzo, "Generalized space-time shift keying 1543 designed for flexible diversity-, multiplexing- and complexity-tradeoffs," 1544 IEEE Trans. Wireless Commun., vol. 10, no. 4, pp. 1144-1153, 1545 Apr. 2011.

[55] H. A. Ngo, C. Xu, S. Sugiura, and L. Hanzo, "Space-time-frequency 1547 shift keying for dispersive channels," IEEE Signal Process. Lett., vol. 18, 1548 no. 3, pp. 177-180, Mar. 2011.

1549

[56] H. A. Ngo and L. Hanzo, "Area spectral efficiency of soft-decision 1550 space-time-frequency shift-keying-aided slow-frequency-hopping mul- 1551 tiple access," IEEE Trans. Veh. Technol., vol. 61, no. 3, pp. 1433-1439, 1552 Mar. 2012.

[57] L. Hanzo, M. Münster, B. J. Choi, and T. Keller, OFDM and MC-CDMA 1554 for Broadband Multi-User Communications, WLANs and Broadcasting. 1555 Hoboken, NJ, USA: Wiley, 2003. 1556

[58] R. Abu-alhiga and H. Haas, "Subcarrier-index modulation OFDM," in 1557 Proc. IEEE Int. Sym. Pers., Indoor Mobile Radio Commun., Tokyo, 1558 Japan, Sep. 2009, pp. 177-181.

[59] E. Başar, Ü. Aygölü, E. Panayırc1, and H. V. Poor, "Orthogonal frequency 1560 division multiplexing with index modulation," in Proc. IEEE Global 1561 Commun. Conf., Anaheim, CA, USA, Dec. 2012, pp. 4741-4746. 1562

[60] R. A. Scholtz, "Multiple access with time-hopping impulse modulation," 1563 in Proc. Mil. Commun. Conf., Boston, MA, USA, Oct. 1993, vol. 2, 1564 pp. $447-450$.

[61] A. Latif and N. D.Gohar, "On the PAPR reduction of hybrid QAM/FSK 1566 (HQFM) OFDM transceiver,” J. Appl. Sci., vol. 8, no. 6, pp. 1061-1066, 1567 2008.

[62] S. Song et al., "A channel hopping technique I: Theoretical studies 1569 on band efficiency and capacity," in Proc. IEEE Int. Conf. Commun., 1570 Circuits Syst., Chengdu, SiChuan, Jun. 2004, vol. 1, pp. 229-233. 1571

[63] R. Y. Mesleh, H. Haas, C. W. Ahn, and S. Yun, "Spatial modulation-A 1572 new low complexity spectral efficiency enhancing technique," in IEEE 1573 Int. Conf. Commun. Netw. China, Beijing, China, Oct. 2006, pp. 1-5. 1574

[64] N. Serafimovski, M. Di Renzo, S. Sinanović, R. Y. Mesleh, and H. Haas, 1575 "Fractional bit encoded spatial modulation (FBE-SM)," IEEE Commun. 1576 Lett., vol. 14, no. 5, pp. 429-431, May 2010. 
[65] M. Di Renzo and H. Haas, "Performance comparison of different spatial modulation schemes in correlated fading channels," in Proc. IEEE Int. Conf. Commun., Cape Town, South Africa, May 2010, pp. 1-6.

[66] Y. Yang and S. Aissa, "Bit-padding information guided channel hopping," IEEE Commun. Lett., vol. 15, no. 2, pp. 163-165, Feb. 2011.

67] Q. Qu, A. Wang, Z. Nie, and J. Zheng, "Block mapping spatial modulation scheme for MIMO systems," J. China Univ. Posts Telecommun., vol. 18, no. 5, pp. 30-36, Oct. 2011.

[68] P. Zhang, D. Yuan, and H. Zhang, "A novel spatial modulation scheme over correlated fading channels," J. Commun., vol. 7, no. 11, pp. 847857, Nov. 2012.

[69] R. Y. Chang, S.-J. Lin, and W.-H. Chung, "Energy efficient transmission over space shift keying modulated MIMO channels," IEEE Trans. Commun., vol. 60, no. 10, pp. 2950-2959, Oct. 2012.

[70] R. Y. Chang, S.-J. Lin, and W.-H. Chung, "New space shift keying modulation with Hamming code-aided constellation design," IEEE Wireless Commun. Lett., vol. 1, no. 1, pp. 2-5, Feb. 2012.

[71] P.-H. Kuo, "SCRAD modulation: High data rate space shift keying scheme permitting reduced-complexity detector," IET Electron. Lett., vol. 48, no. 24, pp. 1567-1569, Nov. 2012.

[72] J. Jeganathan, A. Ghrayeb, and L. Szczecinski, "Spatial modulation: Optimal detection and performance analysis," IEEE Commun. Lett., vol. 12, no. 8, pp. 545-547, Aug. 2008.

[73] S. U. Hwang, S. Jeon, S. Lee, and J. Seo, "Soft-output ML detector for spatial modulation OFDM systems," IEICE Electron. Exp., vol. 6, no. 19, pp. 1426-1431, Sep. 2009.

[74] S. Chen, S. Sugiura, and L. Hanzo, "Semi-blind joint channel estimation and data detection for space-time shift keying systems," IEEE Signal Process. Lett., vol. 17, no. 12, pp. 993-996, Dec. 2010.

[75] M. X. Guo, C. Jia, and Y. H. Shen, "Detection algorithm for spatial modulation system under unconstrained channel," in Proc. IEEE Int. Conf. Commun. Technol., Nanjing, China, Nov. 2010, pp. 458-461.

[76] A. Younis, R. Y. Mesleh, H. Haas, and P. Grant, "Reduced complexity sphere decoder for spatial modulation detection receivers," in Proc. IEEE Global Commun. Conf., Miami, FL, USA, Dec. 2010, pp. $1-5$.

[77] A. Younis, M. Di Renzo, R. Y. Mesleh, and H. Haas, "Sphere decoding for spatial modulation," in Proc. IEEE Int. Conf. Commun., Kyoto, Japan, Jun. 2011, pp. 1-6.

78] P. Yang, Y. Xiao, Q. Tang, B. Zhou, and S. Q. Li, "A low complexity ordered sphere decoding algorithm for spatial modulation," IEICE Trans. Commun., vol. E95-B, no. 07, pp. 2494-2497, Jul. 2012.

[79] A. Younis, S. Sinanovic, M. Di Renzo, R. Y. Mesleh, and H. Haas, "Generalised sphere decoding for spatial modulation," IEEE Trans. Commun, vol. 61, no. 7, pp. 2805-2815, Jul. 2013.

[80] S. Sugiura, C. Xu, S. X. Ng, and L. Hanzo, "Reduced-complexity coherent versus non-coherent QAM-aided space-time shift keying," IEEE Trans. Commun., vol. 59, no. 11, pp. 3090-3101, Nov. 2011.

[81] C. Xu, S. Sugiura, S. X. Ng, and L. Hanzo, "Reduced-complexity softdecision aided space-time shift keying," IEEE Signal Process. Lett., vol. 18 , no. 10, pp. 547-550, Oct. 2011

[82] C. Vladeanu, A. Martian, A. F. Paun, and E. El Assad, "A new ML detector for trellis-coded spatial modulation using hard and soft estimates," in Proc. IEEE Int. Symp. Electron. Telecommun., Timisoara, Romania, Nov. 2012, pp. 143-146.

83] P. Yang, Y. Xiao, L. Li, Q. Tang, and S. Li, "An improved matched-filter based detection algorithm for space-time shift keying systems," IEEE Signal Process. Lett., vol. 19, no. 5, pp. 271-274, May 2012.

[84] H. Xu, "Simple low-complexity detection schemes for $M$-ary quadrature amplitude modulation spatial modulation," IET Commun., vol. 6, no. 17, pp. 2840-2847, Nov. 2012.

[85] N. R. Naidoo, H. Xu, and T. Quazi, "Spatial modulation: Optimal detector asymptotic performance and multiple-stage detection," IET Commun., vol. 5, no. 10, pp. 1368-1376, Jul. 2011.

[86] S. Sugiura, C. Xu, S. X. Ng, and L. Hanzo, "Reduced-complexity iterative-detection aided generalized space-time shift keying," IEEE Trans. Veh. Technol., vol. 61, no. 8, pp. 3656-3664, Oct. 2012.

[87] Q. Tang, Y. Xiao, P. Yang, Q. Yu, and S. Li, "A new low-complexity near-ML detection algorithm for spatial modulation," IEEE Wireless Commun. Lett., vol. 2, no. 1, pp. 90-93, Feb. 2013.

88] C. Xu, S. Sugiura, S. X. Ng, and L. Hanzo, "Spatial modulation and space-time shift keying: Optimal performance at a reduced detection complexity," IEEE Trans. Commun., vol. 61, no. 1, pp. 206-216, Jan. 2013.

[89] J. Wang, S. Jia, and J. Song, "Signal vector based detection scheme for spatial modulation," IEEE Commun. Lett., vol. 16, no. 1, pp. 19-21, Jan. 2012.
[90] J. Zheng, "Signal vector based list detection for spatial modulation," 1655 IEEE Wireless Commun. Lett., vol. 1, no. 4, pp. 265-267, Aug. 2013.

[91] C.-M. Yu et al., "Compressed sensing detector design for space shift key- 1657 ing in MIMO systems," IEEE Commun. Lett., vol. 16, no. 10, pp. 1556- 1658 1559, Oct. 2012.

[92] M. Kadir, S. Sugiura, J. Zhang, S. Cheng, and L. Hanzo, "OFDMA/ 1660 SC-FDMA-aided space-time shift keying for dispersive multiuser sce- 1661 narios," IEEE Trans. Veh. Technol., vol. 62, no. 1, pp. 408-414, 1662 Jan. 2013.

1663

[93] E. Viterbo and J. Boutros, "A universal lattice code decoder for fading 1664 channels," IEEE Trans. Inf. Theory, vol. 45, no. 5, pp. 1639-1642, 1665 Jul. 1999 .

[94] L. Hanzo, T. H. Liew, and B. L. Yeap, Turbo Coding, Turbo Equalisa- 1667 tion, and Space-Time Coding for Transmission Over Fading Channels. 1668 Hoboken, NJ, USA: Wiley, 2002.

[95] B. M. Hochwald and T. L. Marzetta, "Unitary space-time modulation for 1670 multiple-antenna communications in Rayleigh flat fading," IEEE Trans. 1671 Inf. Theory, vol. 46, no. 2, pp. 543-564, Mar. 2000.

1672

[96] B. L. Hughes, "Differential space-time modulation," IEEE Trans. Inf. 1673 Theory, vol. 46, no. 7, pp. 2567-2578, Nov. 2000.

1674

[97] C. Xu, S. Sugiura, S. X. Ng, and L. Hanzo, "Reduced-complexity non- 1675 coherently detected differential space-time shift keying," IEEE Signal 1676 Process. Lett., vol. 18, no. 3, pp. 153-156, Mar. 2011.

1677

[98] L. Xiao, Y. Xiao, P. Yang, and S. Li, "Low-complexity non-coherent 1678 detection for spatial modulation OFDM systems," in Proc. IEEE Int. 1679 Conf. Commun. Technol., Chengdu, China, 2012, pp. 469-473. 1680

[99] R. Zhang and L. Hanzo, "A unified treatment of superposition coding 1681 aided communications: Theory and practice," IEEE Commun. Surveys 1682 Tuts., vol. 13, no. 3, pp. 503-520, Third Quarter 2011.

1683

[100] S. X. Ng and L. Hanzo, "On the MIMO channel capacity of multidimen- 1684 sional signal sets," IEEE Trans. Veh. Technol., vol. 55, no. 2, pp. 528- 1685 536, Mar. 2006.

1686

[101] X. Guan, Y. Xiao, Y. Cai, and W. Yang, "On the mutual information and 1687 precoding for spatial modulation with finite alphabet," IEEE Wireless 1688 Commun. Lett., vol. 2, no. 4, pp. 383-386, Aug. 2013.

[102] J. G. Proakis, Digital Communications, 4th ed. New York, NY, USA: 1690 McGraw-Hill, 2004.

[103] T. Handte, A. Muller, and J. Speidel, "BER analysis and optimization of 1692 generalized spatial modulation in correlated fading channels," in Proc. 1693 IEEE Veh. Technol. Conf., Anchorage, AK, USA, Sep. 2009, pp. 1-5. 1694

[104] P. Yang, Y. Xiao, Y. Yi, and S. Li, "Adaptive spatial modulation for 1695 wireless MIMO transmission systems," IEEE Commun. Lett., vol. 15, 1696 no. 6, pp. 602-604, Jun. 2011. 1697

[105] A. Goldsmith, Wireless Communications. Cambridge, U.K.: 1698 Cambridge Univ. Press, 2005.

[106] M. S. Alaouni and M. K. Simon, Digital Communications Over Fading 1700 Channels, 2nd ed. Hoboken, NJ, USA: Wiley, 2005.

[107] E. Başar, Ü. Aygölü, E. Panayırcı, and H. V. Poor, "Performance of 1702 spatial modulation in the presence of channel estimation errors," IEEE 1703 Commun. Lett., vol. 16, no. 2, pp. 176-179, Feb. 2012.

[108] S. S. Ikki and R. Mesleh, "A general framework for performance analysis 1705 of space shift keying (SSK) modulation in the presence of Gaussian 1706 imperfect estimations," IEEE Commun. Lett., vol. 16, no. 2, pp. 228- 1707 230, Feb. 2012

[109] S. Sugiura and L. Hanzo, "Effects of channel estimation on spatial 1709 modulation," IEEE Signal Process. Lett., vol. 19, no. 12, pp. 805-808, 1710 Dec. 2012.

[110] P. Yang, Y. Xiao, L. Dan, and S. Li, "An improved gradient-based PAPR 1712 reduction method for space shift keying (SSK)-OFDM systems," IEICE 1713 Trans. Commun., vol. E94-B, no. 12, pp. 3532-3539, Dec. 2011.

[111] Z. Hasan, H. Boostanimehr, and V. K. Bhargava, "Green cellular net- 1715 works: A survey, some research issues and challenges," IEEE Commun. 1716 Surveys Tuts., vol. 13, no. 4, pp. 524-540, Nov. 2011.

[112] S. Sugiura and L. Hanzo, "On the joint optimization of dispersion ma- 1718 trices and constellations for near-capacity irregular precoded space-time 1719 shift keying," IEEE Trans. Wireless Commun., vol. 12, no. 1, pp. 380- 1720 387, Jan. 2013.

[113] J. M. Luna-Rivera, D. U. Campos-Delgado, and M. G. Gonzalez-Perez, 1722 "Constellation design for spatial modulation," Procedia Technol., vol. 7, 1723 pp. 71-78, 2013.

[114] M. Maleki, H. R. Bahrami, S. Beygi, M. Kafashan, and N. H. Tran, 1725 "Space modulation with CSI: Constellation design and performance 1726 evaluation," IEEE Trans. Veh. Technol., vol. 62, no. 4, pp. 1623-1634, 1727 May 2013.

[115] P. Yang et al., "Link adaptation for spatial modulation with limited 1729 feedback," IEEE Trans. Veh. Technol., vol. 61, no. 8, pp. 3808-3813, 1730 Oct. 2012 
1732 [116] K. Ishibashi, W. Shin, H. Ochiai, and V. Tarokh, "A peak power efficient 1733 cooperative diversity using star-QAM with coherent/noncoherent detec1734 tion," IEEE Trans. Wireless Commun., vol. 12, no. 5, pp. 2137-2147, 1735 May 2013

1736 [117] L. Lampe, "Noncoherent coded modulation," Ph.D. dissertation, Dept. 1737 Elect. Eng., University of Erlangen, Erlangen, Germany, 2002.

1738 [118] P. Yang et al., "Star-QAM signaling constellations for spatial modula1739 tion," IEEE Trans. Veh. Technol.

1740 [119] P. Yang et al., "Simplified adaptive spatial modulation for limited1741 feedback MIMO,” IEEE Trans. Veh. Technol., vol. 62, no. 6, pp. 26561742 2666, Jul. 2013.

1743 [120] B. M. Mthethwa and H. Xu, "Adaptive $M$-ary quadrature amplitude 1744 spatial modulation," IET Commun., vol. 6, no. 18, pp. 3098-3108, 1745 Dec. 2012.

1746 [121] D. Yang, C. Xu, L.-L. Yang, and L. Hanzo, "Transmit-diversity-assisted 1747 1748

1764 [126] W.-H. Chung and C.-Y. Hung, "Multi-antenna selection using space

1765

1766

1767

1768

1769

1770

1771

1772

1773

1774

1775

1776

1777

1778

1779

1780

1781

1782

1783 [

1784

1785

1786

1787

1788

1789

1790 [134] J. Kim, B. W. Kim, and D. J. Park, "Mitigation of transmit correlation 1791 for TIMO spatial multiplexing through phase rotation," IEEE Commun. 1792 Lett., vol. 12, no. 2, pp. 118-120, Feb. 2008.

1793 [135] A. E. Ekpenyong and Y. Huang, "Feedback constraints for adaptive 1794 transmission," IEEE Signal Process. Mag., vol. 24, no. 3, pp. 69-78, 1795 May 2007

1796 [136] S. Sanayei and A. Nosratinia, "Antenna selection in MIMO systems," 1797 IEEE Commun. Mag., vol. 42, no. 10, pp. 68-73, Oct. 2004.

1798 [137] N. B. Mehta, A. F. Molisch, and S. Kashyap, "Antenna selection in LTE: 1799 From motivation to specification," IEEE Commun. Mag., vol. 50, no. 10, 1800 pp. 144-150, Oct. 2012.

1801 [138] R. W. Heath, Jr., S. Sandhu, and A. Paulraj, "Switching between diversity 1802 and multiplexing in MIMO systems," IEEE Trans. Commun., vol. 53, 1803 no. 6, pp. 962-968, Jun. 2005.

1804 [139] J. N. Laneman, D. N. C. Tse, and G. W. Wornell, "Cooperative diversity 1805 in wireless networks: Efficient protocols and outage behavior," IEEE 1806 Trans. Inf. Theory, vol. 50, no. 12, pp. 3062-3080, Dec. 2004.

1807 [140] S. Sugiura, S. Chen, H. Haas, P. M. Grant, and L. Hanzo, "Coher1808 ent versus non-coherent decode-and-forward relaying aided coopera- tive space-time shift keying," IEEE Trans. Commun., vol. 59, no. 6, 1809 pp. 1707-1719, Jun. 2011.

1810

[141] P. Zhang, S. Chen, and L. Hanzo, "Differential space-time shift keying 1811 aided successive relaying assisted cooperative multiuser CDMA," IEEE 1812 Trans. Veh. Technol., vol. 62, no. 5, pp. 2156-2169, Jun. 2013.

1813

[142] Y. Yang and S. Aissa, "Information-guided transmission in decode- 1814 and forward relaying systems: Spatial exploitation and throughput en- 1815 hancement," IEEE Wireless Commun., vol. 10, no. 7, pp. 2341-2351, 1816 Jul. 2011.

1817

[143] R. Mesleh, S. S. Ikki, E. M. Aggoune, and A. Mansour, "Performance 1818 analysis of space shift keying (SSK) modulation with multiple coopera- 1819 tive relays," EURASIP J. Adv. Signal Process., vol. 2014, no. 1, pp. 1-10, 1820 Sep. 2012.

1821

[144] R. Mesleh, S. S. Ikki, and M. Alwakeel, "Performance analysis of space 1822 shift keying with amplify and forward relaying," IEEE Commun. Lett., 1823 vol. 15, no. 12, pp. 1350-1352, Dec. 2011.

1824

[145] N. Serafimovski, S. Sinanović, M. Di Renzo, and H. Haas, "Dual-hop 1825 spatial modulation (Dh-SM)," in Proc. VTC -Spring, Budapest, Hun- 1826 gary, May 15-18, 2011, pp. 1-5.

[146] S. Narayanan, M. Di Renzo, F. Graziosi, and H. Haas, "Distributed space 1828 shift keying for the uplink of relay-aided cellular networks," in Proc. 1829 IEEE Int. Workshop Comput.-Aided Model. Anal. Des. Commun. Links 1830 Netw., Barcelona, Spain, Sept. 2012, pp. 17-19.

1831

[147] S. Narayanan, M. Di Renzo, F. Graziosi, and H. Haas, "Distributed 1832 spatial modulation for relay networks," in Proc. IEEE Veh. Technol. 1833 Conf._Fall, Las Vegas, USA, Sep. 2013, pp. 130-134. 1834

[148] L. Hanzo, L.-L. Yang, E.-L. Kuan, and K. Yen, Single and Multicarrier 1835 DS-CDMA: Multi-User Detection, Space-Time Spreading, Synchronisa- 1836 tion, Networking, and Standards. Hoboken, NJ, USA: Wiley, 2003. 1837

[149] S. Sugiura, S. X. Ng, L. Kong, S. Chen, and L. Hanzo, "Quasi- 1838 synchronous cooperative networks," IEEE Veh. Technol. Mag., vol. 7, 1839 no. 4, pp. 66-76, Dec. 2012.

1840

[150] H. Wei, L.-L. Yang, and L. Hanzo, "Iterference-free broadband sin- 1841 gle and multicarrier DS-CDMA," IEEE Commun. Mag., vol. 4, no. 3, 1842 pp. 68-73, Feb. 2005.

1843

[151] L. Hanzo, J. Akhtman, L. Wang, and M. Jiang, MIMO-OFDM for 1844 LTE, WiFi and WiMAX: Coherent Versus Non-Coherent and Cooperative 1845 Turbo Transceivers. Hoboken, NJ, USA: Wiley, 2010.

1846

[152] M. I. Kadir, L. Li, S. Chen, and L. Hanzo, "Successive-relaying-aided 1847 decode-and-forward coherent versus noncoherent cooperative multicar- 1848 rier space-time shift keying," IEEE Trans. Veh. Technol., vol. 62, no. 6, 1849 pp. 2544-2557, Jul. 2013.

[153] M. Driusso, F. Babich, M. I. Kadir, and L. Hanzo, "OFDM aided 1851 space-time shift keying for dispersive downlink channels," in IEEE Veh. 1852 Technol. Conf._Fall, Quebec City, QC, QC, Sept. 2012, pp. 1-5. 1853

[154] L. L. Yang, "Signal detection in antenna-hopping space-division 1854 multiple-access systems with space-shift keying modulation," IEEE 1855 Trans. Sig. Process., vol. 60, no. 1, pp. 351-366, Jan. 2012.

[155] P. Som and A. Chockalingam, "Spatial modulation and space shift key- 1857 ing in single carrier communication," in Proc. IEEE PIMRC, Sydney, 1858 NSW, Australia, 2012, pp. 1991-1996.

1859

[156] R. Rajashekar, K. V. S. Hari, and L. Hanzo, "Spatial modulation aided 1860 zero-padded single carrier transmission for dispersive channels," IEEE 1861 Trans. Commun., vol. 61, no. 6, pp. 2318-2329, Jun. 2013.

1862

[157] D. Feng et al., "A survey of energy-efficient wireless communications," 1863 IEEE Commun. Surveys Tuts., vol. 15, no. 1, pp. 167-178, Nov. 2013. 1864

[158] X. Wu, S. Sinanovic, M. Di Renzo, and H. Haas, "Base station energy 1865 consumption for transmission optimised spatial modulation (TOSM) 1866 in correlated channels," in Proc. IEEE 17th Int. Workshop CAMAD, 1867 Barcelona, Spain, 2012, pp. 261-265.

1868

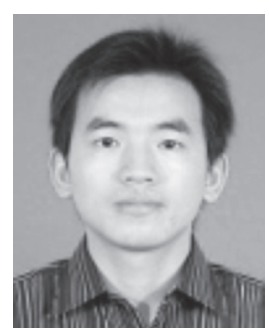

Ping Yang received the B.E., M.E., and Ph.D. de- 1869 grees from the University of Electronic Science and 1870 Technology of China, Chengdu, China, in 2006, 1871 2009 , and 2013, respectively. He is currently with 1872 the National Key Laboratory of Science and Tech- 1873 nology on Communications, University of Electronic 1874 Science and Technology of China. He has published 1875 AQ3 more than 20 international journals and international 1876 conference papers. His research interests include 1877 MIMO systems, space-time coding, and communi- 1878 cation signal processing. 


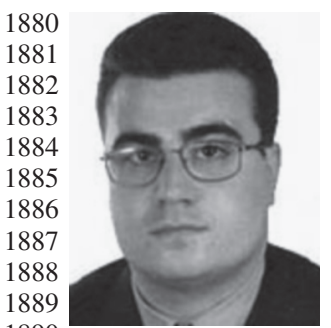

Marco Di Renzo (S'05-AM'07-M'09-SM'14) was born in L'Aquila, Italy, in 1978. He received the Laurea (cum laude) and Ph.D. degrees in electrical and information engineering from the University of L'Aquila, L'Aquila, in 2003 and 2007, respectively, and the Habilitation à Diriger des Recherches (HDR) from the University of Paris-Sud XI, Paris, France, in 2013.

Since January 2010, he has been a Tenured Academic Researcher ("Chargé de Recherche Titulaire") with the French National Center for Scientific Re1891 search (CNRS), as well as a faculty member of the Laboratory of Signals 1892 and Systems (L2S), a joint research laboratory of the CNRS, the École 1893 Supérieure d'Électricité (SUPÉLEC), and the University of Paris-Sud XI. His 1894 main research interests are in the area of wireless communications theory.

1895 Dr. Di Renzo currently serves an Editor of the IEEE COMMUNICATIONS 1896 LETTERS and of the IEEE TRANSACTIONS ON COMMUNICATIONS (Wireless 1897 Communications-Heterogeneous Networks Modeling and Analysis). He was 1898 the recipient of a special mention for the outstanding five-year (1997-2003) 1899 academic career, University of L'Aquila; the THALES Communications fel1900 lowship (2003-2006), University of L'Aquila; the 2004 Best Spin-Off Com1901 pany Award, Abruzzo Region, Italy; the 2006 DEWS Travel Grant Award, 1902 University of L'Aquila; the 2008 Torres Quevedo Award, Ministry of Sci1903 ence and Innovation, Spain; the "Dérogation pour l'Encadrement de Thèse" 1904 (2010), University of Paris-Sud XI; the 2012 IEEE CAMAD Best Paper 1905 Award; the 2012 IEEE WIRELESS COMMUNICATIONS LETTERS Exemplary 1906 Reviewer Award; the 2013 IEEE VTC-Fall Best Student Paper Award; the 19072013 Network of Excellence NEWCOM\# Best Paper Award; the 2013 IEEE 1908 Transactions on Vehicular Technology Top Reviewer Award; the 19092013 IEEE COMSOC Best Young Researcher Award for Europe, Middle East, 1910 and Africa (EMEA Region); and the 2014 IEEE ICNC Single Best Paper Award 1911 Nomination (Wireless Communications Symposium).

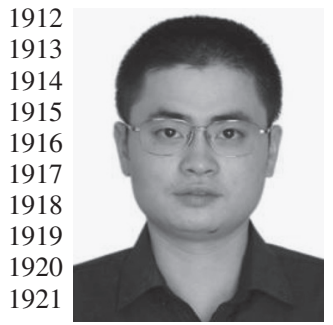

Yue Xiao received the Ph.D. degree in communication and information systems from the University of Electronic Science and Technology of China, Chengdu, China, in 2007. He is currently an Associate Professor with University of Electronic Science and Technology of China. He has published more than 30 international journals and been involved in several projects in Chinese Beyond 3G Communication R\&D Program. His research interests are in the area of wireless and mobile communications.

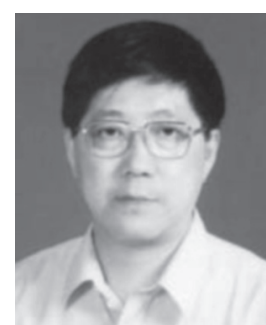

Shaoqian $\mathbf{L i}$ received the B.Eng. degree in electrical 1922 engineering from the American University of Beirut, 1923 Beirut, Lebanon, in 2004 and the M.Sc. degree 1924 in radio frequency communication systems and the 1925 $\mathrm{Ph} . \mathrm{D}$. degree in wireless communications from the 1926 University of Southampton, Southampton, U.K., in 1927 2005 and 2008, respectively. Following his Ph.D. 1928 degree, he joined Imagination Technologies as a Re- 1929 search Engineer, where he worked on designing and 1930 developing the BICM peripherals in Imagination's 1931 multi-standard communications platform, which re- 1932 sulted in several patent applications. In January 2012, he joined the School of 1933 Electronics and Computer Science, University of Southampton, as a Lecturer 1934 in the Communications, Signal Processing and Control Research Group. He 1935 has published a Wiley-IEEE book and in excess of 40 journal and international 1936 conference papers. His research interests include machine-to-machine commu- 1937 nications, millimeter-wave communications, large-scale MIMO, cooperative 1938 communications, and radio over fiber systems. Dr. Li was the recipient of 1939 several academic awards.

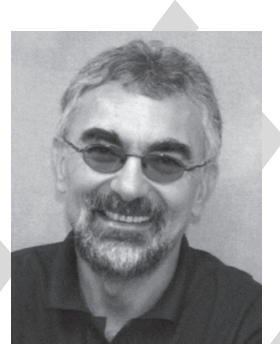

Lajos Hanzo received the M.S. degree in electronics 1941 and the Ph.D. degree from the Technical University 1942 of Budapest, Budapest, Hungary, in 1976 and 1983, 1943 respectively; the D.Sc. degree from the University of 1944 Southampton, Southampton, U.K., in 2004; and the 1945 "Doctor Honoris Causa" degree from the Technical 1946 University of Budapest in 2009.

During his 37-year career in telecommunications, 1948 he has held various research and academic posts in 1949 Hungary, Germany, and the U.K. Since 1986, he has 1950 been with the School of Electronics and Computer 1951 Science, University of Southampton, U.K., where he holds the Chair in 1952 Telecommunications. He is currently directing a 100-strong academic research 1953 team, working on a range of research projects in the field of wireless multimedia 1954 communications sponsored by industry, the Engineering and Physical Sciences 1955 Research Council (EPSRC) U.K., the European Research Council's Advanced 1956 Fellow Grant, and the Royal Society's Wolfson Research Merit Award. During 1957 2008-2012, he was a Chaired Professor with Tsinghua University, Beijing 1958 China. He is an enthusiastic supporter of industrial and academic liaison and 1959 offers a range of industrial courses. He has successfully supervised more than 1960 80 Ph.D. students, coauthored 20 John Wiley/IEEE Press books on mobile 1961 radio communications totaling in excess of 10000 pages, and published more 1962 than 1400 research entries at IEEE Xplore. He has more than 19000 citations. 1963 His research is funded by the European Research Council's Senior Research 1964 Fellow Grant. For further information on research in progress and associated 1965 publications, please refer to http://www-mobile.ecs.soton.ac.uk.

Dr. Hanzo is a Fellow of the Royal Academy of Engineering, the Institution 1967 of Engineering and Technology, and the European Association for Signal 1968 Processing. He is also a Governor of the IEEE Vehicular Technology Society. 1969 During 2008-2012, he was the Editor-in-Chief of the IEEE Press. He has served 1970 as the Technical Program Committee Chair and the General Chair of IEEE 1971 conferences, has presented keynote lectures, and has been awarded a number 1972 of distinctions. 


\section{AUTHOR QUERIES}

\section{AUTHOR PLEASE ANSWER ALL QUERIES}

AQ1 = Please provide publication update in Ref. [118].

AQ2 = Please provide page range in Ref. [124].

AQ3 = This was added as given in the author's affiliation information. Is this correct? If not, please provide his current affiliation data.

\section{END OF ALL QUERIES}




\title{
Design Guidelines for Spatial Modulation
}

\author{
Ping Yang, Marco Di Renzo, Senior Member, IEEE, Yue Xiao, Shaoqian Li, Senior Member, IEEE, and
}

Lajos Hanzo, Fellow, IEEE

\begin{abstract}
4 Abstract-A new class of low-complexity, yet energy-efficient 5 Multiple-Input Multiple-Output (MIMO) transmission tech6 niques, namely, the family of Spatial Modulation (SM) aided 7 MIMOs (SM-MIMO), has emerged. These systems are capable of 8 exploiting the spatial dimensions (i.e., the antenna indices) as an 9 additional dimension invoked for transmitting information, apart 10 from the traditional Amplitude and Phase Modulation (APM). SM 11 is capable of efficiently operating in diverse MIMO configurations 12 in the context of future communication systems. It constitutes a 13 promising transmission candidate for large-scale MIMO design 14 and for the indoor optical wireless communication while relying 15 on a single-Radio Frequency (RF) chain. Moreover, SM may be 16 also viewed as an entirely new hybrid modulation scheme, which is 17 still in its infancy. This paper aims for providing a general survey 18 of the SM design framework as well as of its intrinsic limits. In 19 particular, we focus our attention on the associated transceiver 20 design, on spatial constellation optimization, on link adaptation 21 techniques, on distributed/cooperative protocol design issues, and 22 on their meritorious variants.
\end{abstract}

23 Index Terms-Cooperative communications, large-scale 24 MIMO, link adaptation, space-time coding, spatial modulation.

\section{INTRODUCTION}

26 ULTIPLE-INPUT multiple-output (MIMO) systems are 27 capable of achieving a capacity gain and/or diversity 28 gain, which is based on striking a beneficial trade-off, de29 pending on the near-instantaneous channel conditions [1]-[4]. 30 Hence they have been adopted in most of the recent com31 munication standards, such as IEEE 802.11n, IEEE 802.16e, 32 and 3GPP Long-Term Evolution (LTE) [5], [6]. In a wireless 33 MIMO transmission system, the transmission technique em34 ployed plays an important role in determining the achievable 35 system performance. Recently, the conventional spatial-domain 36 MIMO transmission techniques have been extended to the time37 domain, the frequency-domain as well as to their combinations

Manuscript received October 15, 2013; revised March 19, 2014; accepted May 23, 2014. This work was supported by the National Science Foundation of China under Grant 61101101, by the European Research Council's Advanced Fellow Grant, by the National Basic Research Program of China under Grant 2013CB329001, and by the European Commission under the auspices of the FP7-PEOPLE ITN-GREENET Project under Grant 264759.

P. Yang, Y. Xiao, and S. Li are with the National Key Laboratory of Science and Technology on Communications, University of Electronic Science and Technology of China, Chengdu 611731, China (e-mail: yplxw@163.com; 1sq@uestc.edu.cn; xiaoyue@uestc.edu.cn).

M. Di Renzo is with the Laboratory of Signals and Systems (L2S), French National Center for Scientific Research (CNRS), University of Paris-Sud XI, 91192 Gif-sur-Yvette, France (e-mail: marco.direnzo@1ss.supelec.fr).

L. Hanzo is with the School of Electronics and Computer Science, University of Southampton, Southampton SO17 1BJ, U.K. (e-mail: 1h@ecs.soton.ac.uk).

Color versions of one or more of the figures in this paper are available online at http://ieeexplore.ieee.org.

Digital Object Identifier 10.1109/COMST.2014.2327066
[7], [8]. In order to efficiently exploit the associated grade of 38 freedom offered by MIMO channels, a meritorious transmis- 39 sion technique should be designed to satisfy a diverse range 40 of practical requirements and to strike an attractive tradeoff 41 amongst the conflicting factors of the computational complexity 42 imposed, the attainable bit error ratio (BER) and the achievable 43 transmission rate [9], [10].

In the diverse family of MIMO techniques, the recently pro- 45 posed spatial modulation (SM) [11] (which was referred to as 46 Information-Guided Channel Hopping (IGCH) modulation in 47 [12]) is particularly promising, since it is capable of exploiting 48 the indices of the transmit antennas (TAs) as an additional 49 dimension invoked for transmitting information, apart from 50 the traditional Amplitude and Phase Modulation (APM) [13]. 51 At a given Signal to Noise Ratio (SNR), the throughput of 52 the SM-MIMO may potentially become higher than that of 53 Space-Time Coding (STC) [14], but this is not necessarily its 54 most prominent benefit, because in SM only a single TA is 55 activated at any time instant. Hence SM is capable of dispensing 56 with the requirement of multiple Radio Frequency (RF) chains, 57 therefore relaxing the Inter-Antenna-Synchronization (IAS) 58 specifications, whilst mitigating the Inter Antenna Interference 59 (IAI) of conventional MIMO techniques [15]. Additionally, 60 the single-RF design is capable of reducing the total power 61 consumption. In fact, only a single power amplifier is needed 62 for implementing SM-MIMO systems, which is typically re- 63 sponsible for the vast majority of power dissipation at the 64 transmitter [16], [17]. Another advantage of SM is that it may 65 be flexibly configured for diverse transmit and receive antenna 66 constellations, especially for the challenging scenario of asym- 67 metric/unbalanced MIMO systems, whose channel matrix is 68 rank-deficient [15].

Due to the above-mentioned advantages, SM constitutes an 70 attractive option for the emerging family of large-scale MIMO 71 systems [18], [19]. As a further advance, the principle of 72 SMs was also extended to indoor optical wireless communi- 73 cation in [20]-[23], which relies on optical transmissions for 74 conveying information. Altogether, SM constitutes a promis- 75 ing low-complexity energy-efficient MIMO transmission tech- 76 nique, which relies on a low-cost transceiver and is capable 77 of efficiently operating in diverse MIMO configurations in 78 the context of future communication systems. Recently, the 79 potential benefits of SM have been validated not only via 80 simulations [11], [14] but also by experiments [24]-[26]. The 81 benefits of SM-MIMOs aided wireless communications are 82 summarized in Fig. 1. In the sequel, they are characterized in 83 more detail.

The wide-ranging simulation based and analytical studies 85 disseminated in [27]-[34] have characterized some of the 86 


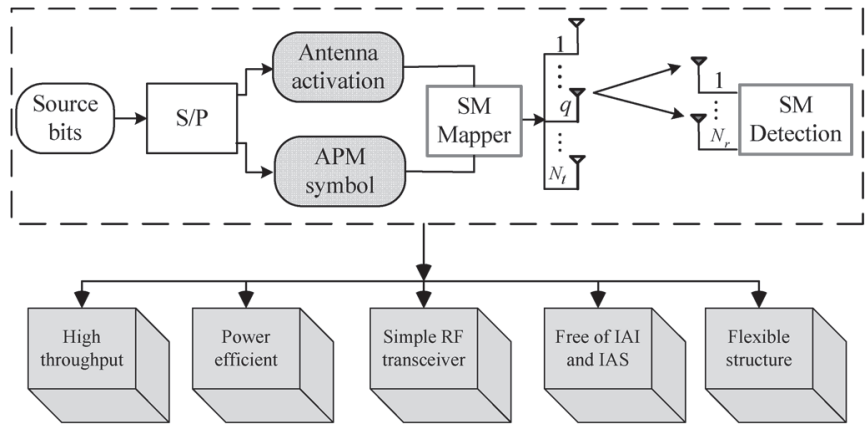

Fig. 1. Benefits of SM-MIMOs for wireless communications.

87 fundamental properties of SM related to the channel's corre88 lation [27], [28]. Furthermore, the issues of achieving transmit 89 diversity [29], the effects of power imbalance [30], the specific 90 choice of the APM scheme used [31], the impact of the specific 91 channel encountered [29], [32] as well as the effects of channel 92 estimation errors [33], [34] were also characterized. It was 93 found that the performance of SM-MIMOs is highly dependent 94 on the specific type of the APM scheme used. For example, as 95 a hybrid modulation scheme, which combines the classic APM 96 constellation and the spatial-domain (SD) constellation, the 97 SM's achievable performance depends both on the minimum 98 Euclidean distance (ED) of the APM constellation employed, 99 as well as the on absolute values of the modulated symbols 100 [29]. Hence, a suitable APM scheme has to be carefully de101 signed for exploiting the benefits of this hybrid modulation 102 scheme.

103 On the other hand, it was also noted that the conventional 104 open-loop SM schemes [11], [12] only offer receive-diversity 105 gains. Hence there is also a paucity of SM-MIMO solutions on 106 how to increase the system's robustness to time-varying channel 107 conditions with the aid of either open or closed-loop transmit108 symbol design techniques [14]. Additionally, unlike in conven109 tional MIMO techniques, the transmit vectors of SM-MIMO 110 schemes are sparsely populated, since they have mostly zero 111 values [11]. This constraint makes SM rather different from 112 classic Space Time Block Codes (STBC) [35] designed for 113 achieving a diversity gain or from Spatial Division Multiplexing 114 (SDM) [36] conceived for attaining a multiplexing gain as well 115 as from the hybrid SDM-STBC schemes [37] aiming for strik116 ing a compromise. In order to increase the robustness of SM117 MIMO systems, the classic time-variant parameter adaptation 118 techniques [38], such as power allocation and precoding [39]119 [41], which were proposed for conventional MIMO techniques 120 may not be directly applied to SM schemes owing to their 121 specific transmission mode.

122 In this treatise, we provide a general survey of the SM design 123 framework as well as of its intrinsic limitations. We summarize 124 the most recent research achievements and outline their poten125 tial applications, as well as their impediments, which have to be 126 overcome before these MIMO technique may be used as main127 stream solutions in practical systems. In particular, we focus 128 our attention on the associated transceiver design, on spatial 129 constellation optimization, on link adaptation techniques, on 130 distributed/cooperative protocol design and on their meritorious 131 variants.
The paper is organized as follows. Section II reviews the con- 132 ventional SM technique and its relevant variants, emphasizing 133 the flexible transceiver design techniques conceived for striking 134 an attractive trade-off amongst the often conflicting system 135 requirements. The spatial constellation optimization and the as- 136 sociated link adaptation techniques are presented in Sections III 137 and IV, respectively. Section V surveys the family of relay 138 aided SM schemes, which exploits the particular information 139 transmission characteristics of SM and introduces the class of 140 SM-related systems designed for dispersive channels. Finally, 141 Section VI concludes the paper.

142

Although the list of the references is not exhaustive, the 143 papers cited as well as the references therein can serve as 144 a good starting point for further reading. In particular, there 145 are several tutorial-style articles, [8], [14] and [15], which 146 tend to have quite a different focus. To be specific, in [8], 147 the authors have reviewed diverse MIMO arrangements and 148 then focus on a new class of MIMOs based on the concept 149 of space-time shift keying. In [14], the authors have evaluated 150 the advantages and disadvantages of SM with respect to other 151 popular MIMO schemes and summarized some early research 152 achievements. Moreover, in [15], some of the co-authors of 153 this treatise have provided a comprehensive survey of spa- 154 tial modulation research, with an emphasis on a generalized 155 transceiver scheme combining spatial modulation with spatial 156 multiplexing and space-time block coding in order to increase 157 either the spectral efficiency or the diversity gain. The price to 158 pay for this flexibility is the need for multiple radio frequency 159 chains. Moreover, in [15] the authors emphasized the energy 160 efficiency of MIMO-based transmission schemes and the first 161 SM-MIMO-based testbed results recorded both in realistic 162 outdoor and indoor propagation environments were reported. 163 Suffice to say that [15] was conceived for stimulating cross- 164 disciplinary research across different communities, whilst this 165 contribution is targeted at readers with a background in wireless 166 communications, who might like to delve into SM-research. 167

Against this background, this contribution firstly provides a 168 succinct description of the basic spatial modulation principle. 169 To be specific, the SM techniques are classified and then the 170 corresponding detection techniques are categorized with the aid 171 of tables for explicit clarity. Moreover, this paper is more fo- 172 cused on illustrating those results that lead to new design guide- 173 lines, as exemplified by the constellation optimization issues 174 of SM. Furthermore, there is a special emphasis on powerful 175 adaptive modulation aided SM and on precoding aided SM. 176 A range of performance metrics are introduced for optimizing 177 spatial modulation, which rely either on the available long-term 178 statistical or on the near-instantaneous knowledge about the 179 channel.

\section{TRAnsceiver Design of SM-MIMO}

\section{A. The Transmitter Design of SM}

In this section, we consider the $\left(N_{t} \times N_{r}\right)$-element SM- 183 MIMO system, which relies on $N_{t}$ transmit and $N_{r}$ receive 184 antennas, while communicating over frequency-flat Rayleigh 185 fading channels. The conventional bit-to-symbol mapping rule 186 


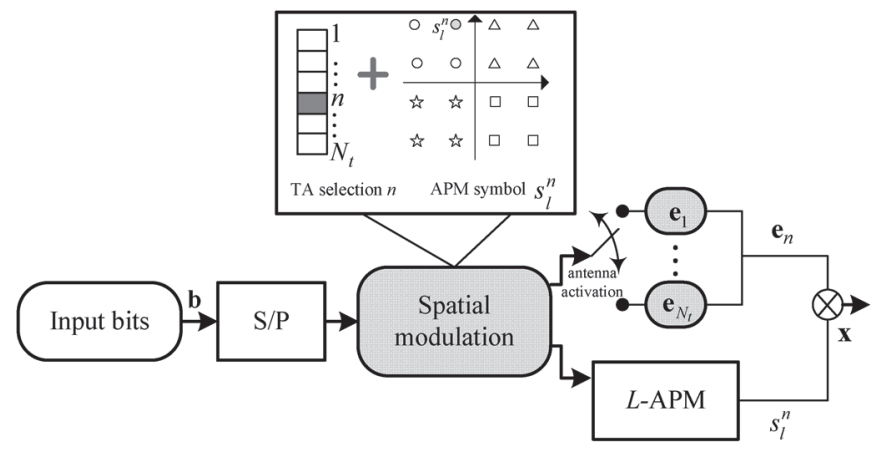

Fig. 2. SM bit-to-symbol mapping rule.

187 [11] of SM is portrayed in Fig. 2, which can be divided into 188 three steps as follows:

189 Algorithm 1: Bit-to-symbol mapping principle of the SM 190 transmitter of Fig. 2

191 1) First, the information bit stream is divided into vectors containing $m_{\text {all }}=\log _{2}\left(L \cdot N_{t}\right)$ bits each.

2) Next, each vector is further split into two sub-vectors of $\log _{2}\left(N_{t}\right)$ and $\log _{2}(L)$ bits each. The bits in the first subvector are used for activating a unique TA for transmission, while the bits in the second sub-vector are mapped to an APM symbol $s_{l}^{n}$. Note that the TA activation process can be described by the $N_{t}$-dimensional standard basis vector $\mathbf{e}_{n}\left(1 \leq n \leq N_{t}\right)$ (i.e., $\mathbf{e}_{1}=[1,0, \cdots, 0]^{T}$ ).

3) Finally, the transmitted symbol $x$ is comprised of the APM symbol $s_{l}^{n}$ emitted from the activated TA $n$. The resultant modulated symbol can be formulated as $\mathbf{x}=$ $s_{l}^{q} \mathbf{e}_{q} \in \mathbb{C}^{N_{t} \times 1}$.

204 The corresponding vector-based signal received at the SM205 MIMO receiver is given by

$$
\mathbf{y}=\mathbf{H x}+\mathbf{n}=\mathbf{h}_{n} s_{l}^{n}+\mathbf{n}
$$

206 where $\mathbf{H}$ is an $\left(N_{r} \times N_{t}\right)$-element channel matrix, $\mathbf{h}_{n}$ is the 207 nth column of $\mathbf{H}$ and the elements of the $N_{r}$-dimensional 208 noise vector $\mathbf{n}$ are complex Gaussian random variables obeying $209 \mathcal{C N}\left(0, N_{0}\right)$.

\section{B. Variants of the SM Principle}

211 The first conference paper on SM was published in 2001 [45], 212 but its extensive research was mainly fueled by the pioneering 213 works of Haas et al. [42], Mesleh et al. [11], followed by 214 Sugiura et al. [43], Yang et al. [12] and Jeganathan et al. [44]. 215 Throughout its decade-long history, the SM concept has been 216 termed in different ways and it was extended to different scenar217 ios. A range of major contributions on the subject of SM and its 218 related variants are listed in Table I. Specifically, the concept of 219 SM was first touched upon in [45], where the distinct multipath 220 components were exploited for detection. In [42], a novel Or221 thogonal Spatial-Division Multiplexing (OSDM) scheme was proposed, which utilizes the index of the TAs as a means of 222 conveying additional source information. In [11], a beneficial 223 framework was established for the bit-to-symbol mapping rule 224 of SM. It was also demonstrated in [11] that SM may be 225 capable of attaining a better performance than other conven- 226 tional MIMO schemes, such as the Vertical Bell Laboratories 227 Layered Space-Time (V-BLAST) and STBC [4], even with- 228 out reducing the achievable data rate,. The above-mentioned 229 IGCH technique was proposed in [12] for achieving a high 230 throughput. Later, Space Shift Keying (SSK) [44] modulation 231 was conceived for relying exclusively on the TA indices to 232 convey information, whilst entirely dispensing with any classic 233 Phase Shift Keying (PSK)/Quadrature Amplitude Modulation 234 (QAM) signaling [13]. In a nutshell, all of the above-mentioned 235 schemes activate only a single TA at any instant in order to 236 maintain a low complexity, whilst mitigating to IAI and IAS 237 specifications, as well as reducing to total power consumed. 238

Motivated by the above concepts, various generalized ver- 239 sions of SM were proposed. First, as a natural extension of 240 SSK, the Generalized SSK (GSSK) scheme was proposed in 241 [46], which activates multiple TAs for the sake of achieving 242 an increased-rate data transmission. This extension has also 243 been incorporated into the SM scheme and two classes of 244 Generalized SM (GSM) schemes were obtained [47]-[49]. To 245 be specific, in [47] a class of GSM arrangements was proposed 246 for the sake of attaining increased transmit diversity gains, 247 which uses all the active TAs for transmitting the same APM- 248 modulated symbols. By contrast, in [48] and [49], another class 249 of GSM arrangements was proposed for attaining an increased 250 multiplexing gain, which uses the active transmit antennas to 251 carry different information symbols during each time slot. Note 252 that the above-mentioned generalized SM schemes of [46]- 253 [49] allow us to activate several-rather than only a single 254 antenna-at the transmitter for bit-to-symbol mapping, hence 255 they are capable of overcoming a specific constraint of SM, 256 namely that the number of TAs has to be a power of two. 257 Moreover, SM was combined with the classic STBC scheme 258 in [50] and with Trellis Coding (TC) in [51]-[53] in order to 259 take advantage of the benefits of both.

260

Recently, Space-Time Shift Keying (STSK) [43] and its 261 generalized form, namely GSTSK [54] was further extended by 262 applying SSK/SM to both the space and to the time dimensions 263 upon combining SSK/SM with space-time block codes, which 264 resulted in an improved diversity versus multiplexing tradeoff. 265 In contrast to the TA-index of conventional SM, in STSK [43], 266 the specific indices of the pre-designed space-time dispersion 267 matrices were exploited for conveying additional data. To be 268 specific, one out of $N_{t}$ dispersion matrices was activated rather 269 than simply activating one out of $N_{t}$ TAs in order to disperse a 270 PSK/QAM symbol in STSK, where a beneficial diversity gain 271 may be achieved as a merit of the simultaneous transmissions 272 from the multiple TAs. As a further advance, the STSK concept 273 was extended to the frequency domain in [55] and [56] with 274 the assistance of a Frequency-Shift Keying (FSK) modulator. 275 To be specific, in [55] the Space-Frequency Shift Keying 276 (SFSK) as well as the Space-Time-Frequency Shift Keying 277 (STFSK) schemes were proposed, which have the added benefit 278 of spreading the transmit signal across both the space and time 279 
TABLE I

CONTRIBUtion to SM SCHEME AND ITS RELATED VARIANTS

\begin{tabular}{|c|c|c|}
\hline Year & Authors & Contributions \\
\hline 2001 & "Chau and $\mathrm{Yu}[45]$ & $\begin{array}{l}\text { Introduced the concept of SM and exploited the distinct multipath fading } \\
\text { characteristics for antenna index detection. }\end{array}$ \\
\hline 2002 & Haas et al. $[42]$ & $\begin{array}{l}\text { Proposed an OSDM scheme, which uses Walsh-Hadamard codes and an antenna } \\
\text { array for data multiplexing. }\end{array}$ \\
\hline 2004 & Song et al. [62] & $\begin{array}{l}\text { Proposed channel hopping modulation, which is applicable to an arbitrary number } \\
\text { of TAs. }\end{array}$ \\
\hline 2006 & Mesleh et al. [63] & $\begin{array}{l}\text { Proposed an efficient MIMO scheme, namely SM, which maps multiple information } \\
\text { bits into a single information symbol and to the index of a single TA } \\
\text { transmitting antenna. }\end{array}$ \\
\hline \multirow[t]{3}{*}{2008} & Jeganathan et al. [46] & $\begin{array}{l}\text { Conceived an SSK concept and its improved version of the SSK modulation, } \\
\text { namely GSSK, which activates multiple TAs for data transmission. }\end{array}$ \\
\hline & Yang et al. [12] & $\begin{array}{l}\text { Introduced the IGCH technique based on the fact that the independent fading of } \\
\text { multiple channel can be used as an additional information channel. }\end{array}$ \\
\hline & Mesleh et al. [11] & $\begin{array}{l}\text { Proposed a simple MRC-based receiver design for SM, which detects the TA } \\
\text { index and APM separately. }\end{array}$ \\
\hline \multirow[t]{2}{*}{2009} & Abu-alhiga et al. [58] & $\begin{array}{l}\text { Designed a power-efficient SIM scheme, which maps a stream of bits into the indices } \\
\text { of the available subcarriers in an on-off keying fashion. }\end{array}$ \\
\hline & Jeganathan et al. [44] & $\begin{array}{l}\text { Presented the framework of SSK, which is a low-complexity version of SM } \\
\text { concept and exclusively employs the TA indices for data transmission. }\end{array}$ \\
\hline \multirow[t]{7}{*}{2010} & Di Renzo et al. [30] & $\begin{array}{l}\text { Introduced an opportunistic power allocation scheme for SSK modulation, which } \\
\text { exploits CSI for performance improvement }\end{array}$ \\
\hline & Mesleh et al. [51] & $\begin{array}{l}\text { Proposed a trellis coded SM (TC-SM) scheme, where the Trellis Coded Modulation } \\
\text { is applied to SM to improve its performance in correlated channels. }\end{array}$ \\
\hline & Serafimovski et al. [64] & $\begin{array}{l}\text { Introduced a Fractional Bit Encoded (FBE)-SM scheme, which allows the } \\
\text { transmitter to be equipped with an arbitrary number of TAs. }\end{array}$ \\
\hline & Fu et al. $[47]$ & $\begin{array}{l}\text { Proposed high-rate generalized SM, which uses multiple active TAs to encode } \\
\text { information bits. }\end{array}$ \\
\hline & Younis et al. [48] & $\begin{array}{l}\text { Proposed a GSM scheme, which sends the same symbol from more than one } \\
\text { transmit antenna at a time. }\end{array}$ \\
\hline & Sugiura et al. [43] & $\begin{array}{l}\text { A novel STSK modulation scheme is proposed, which constitutes a generalized shift } \\
\text { keying architecture utilizing both the space as well as time dimensions and hence } \\
\text { includes the SM and SSK schemes as special cases. }\end{array}$ \\
\hline & Renzo et al. [65] & $\begin{array}{l}\text { Introduced the Time-Orthogonal Signal Design assisted SM (TOSD-SM) for offering } \\
\text { transmit-diversity. }\end{array}$ \\
\hline \multirow[t]{6}{*}{2011} & Yang et al. [66] & $\begin{array}{l}\text { Designed a Bit-Padding IGCH (BP-IGCH) scheme, which eliminates the limitation } \\
\text { that the number of TAs has to be a power of two based on the IGCH concept. }\end{array}$ \\
\hline & Başar et al. $[50]$ & $\begin{array}{l}\text { Combined SM and STBC to take advantage of the benefits of both, while avoiding } \\
\text { their drawbacks. }\end{array}$ \\
\hline & Sugiura et al. $[54]$ & $\begin{array}{l}\text { Proposed a novel Generalized STSK (G-STSK) architecture for striking a flexible } \\
\text { tradeoff among diversity, throughput as well as complexity. }\end{array}$ \\
\hline & Ngo et al. [55] & $\begin{array}{l}\text { Proposed the SFSK modulation as well as the STFSK concept, which spreads the } \\
\text { transmit signal across the space- and time- and frequency-domain. }\end{array}$ \\
\hline & Qu et al. [67] & Conceived a block mapping SM (BMSM) scheme for increasing the transmit rate. \\
\hline & Başar et al. [52] & Proposed a new TC-SM scheme with for achieving higher diversity and coding gains. \\
\hline \multirow[t]{4}{*}{2012} & Zhang et al. [68] & $\begin{array}{l}\text { Introduced a novel SM scheme based on Ungerboeck's set partitioning for a } \\
\text { correlated Rician fading scenario. }\end{array}$ \\
\hline & Wang et al. $[49]$ & $\begin{array}{l}\text { Designed a novel high-rate Multiple Active-SM (MA-SM) schemes and a } \\
\text { near-optimal decoder with linear complexity. }\end{array}$ \\
\hline & Chang et al. $[69],[70]$ & $\begin{array}{l}\text { Proposed a new SSK modulation with Hamming code-aided constellation design for } \\
\text { striking a flexible tradeoff among transmission rate, performance and power. }\end{array}$ \\
\hline & Kuo $[71]$ & $\begin{array}{l}\text { Proposed a Symbol Coordinate Representations in Antenna Domains modulation } \\
\text {, which leads superior performance to both SM and GSSK at the same data rate. }\end{array}$ \\
\hline \multirow[t]{3}{*}{2013} & Di Renzo et al. [15] & $\begin{array}{l}\text { Illustrated the archived experimental results substantiating the benefits of SM } \\
\text { and presented its beneficial application areas. }\end{array}$ \\
\hline & Serafimovski et al. $[26]$ & First practical testbed implementation of SM in indoors (laboratory environment). \\
\hline & Younis et al. [25] & luation of SM in indoors using real-world measured channels. \\
\hline
\end{tabular}

280 domains, as well as the frequency domain. In [56], the STFSK 281 concept was extended to the Slow-Frequency-Hopping Multi282 ple Access (SFHMA) philosophy for the sake of supporting 283 multiple users and its Area Spectral Efficiency (ASE) gain over 284 the classic Gaussian Minimum Shift Keying (GMSK)-aided 285 SFHMA and GMSK assisted time-division/frequency-division 286 multiple access (TD/FDMA) systems was quantified.

287 Inspired by the concept of SM/SSK, the subcarrier orthogo288 nality can also be exploited and the indices of active subcarriers 289 of Orthogonal Frequency-Division Multiplexing (OFDM) [57] 290 symbols can be employed for conveying additional information, 291 which is referred to as Subcarrier-Index Modulation (SIM)
[58]. Based on the same principle, but following a different 292 approach from that of [58], a novel transmission scheme termed 293 as OFDM combined with Index Modulation (OFDM-IM) was 294 proposed in [59] for frequency selective fading channels, with 295 the objective of increasing the data rate as well as simultane- 296 ously improving the attainable BER performance. In Fig. 3, 297 we classify the above-mentioned schemes, which exploit dif- 298 ferent degrees of freedom offered by the temporal domain, 299 frequency domain and spatial domain fading. For completeness, 300 we also briefly allude to the classic time hopping impulse 301 modulation (THIM) [60], which exploits the indices of time- 302 slots for implicitly conveying additional data. As a further 303 


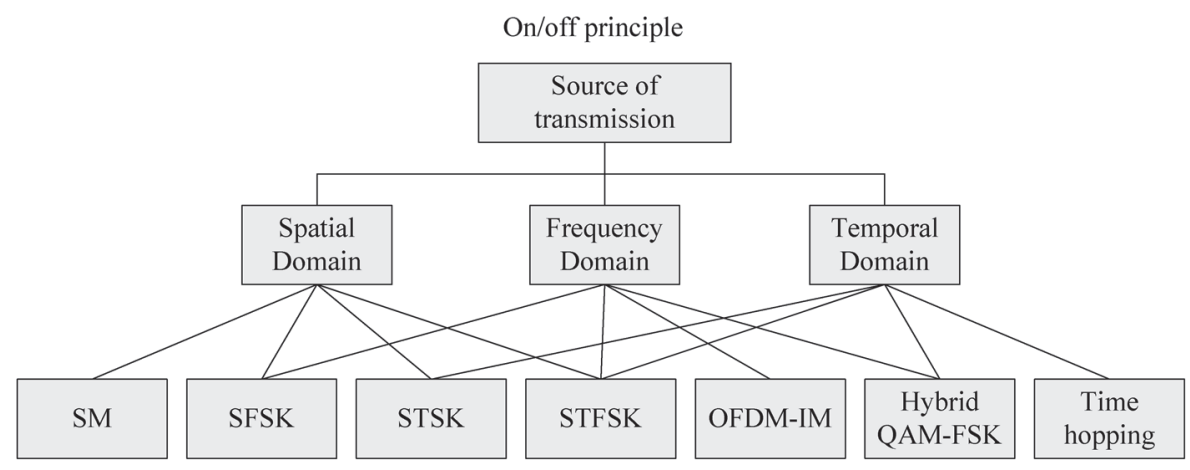

Fig. 3. Transmission techniques based on the on/off keying principle applied to the temporal domain, frequency domain and spatial domain. Here, we have "SM": spatial modulation, "SFSK": Space-Frequency Shift Keying, "STSK": Space-Time Shift Keying, "STFSK": Space-Time-Frequency Shift Keying, and "OFDM-IM": OFDM with Index Modulation.

304 improvement, hybrid QAM-FSK modulation [61] combine 305 the time-frequency domain for the sake of exploiting their 306 independent fading.

\section{C. Detector Design}

308 As seen in Fig. 2, the TA index is combined with the 309 APM symbol index by the SM mapper. Hence, only the TA 310 antenna index and the transmitted APM symbol index have to 311 be estimated at the receiver. Note that most variants of SM, such 312 as STSK and SSK, have an equivalent system model similar to 313 (1), which is free from the effects of ICI, and each equivalent 314 transmit vector includes only a single non-zero component [43], 315 [44]. As a result, they may be able to use the same detection 316 algorithm. As indicated in [72]-[88], the detection techniques 317 of SM-MIMO systems may be broadly divided into four funda318 mental categories: Maximum Likelihood (ML) detection [72]319 [74], Matched Filter (MF) based detection [11], [75], Sphere 320 Decoding (SD) algorithm based detection [76]-[79] and hybrid 321 detection, which combines the modified MF concept and the 322 reduced-complexity exhaustive ML search of [12], [80]-[88]. 323 An overview of the various detection techniques conceived 324 for SM-related schemes is seen in Fig. 4. Next, they will be 325 characterized in more detail.

326 An optimal ML-based SM detector, which carries out an 327 exhaustive search for the global optimum in the entire signal 328 space, was developed in [72]. This detector jointly detects the 329 active TA index as well as the transmitted APM symbol and 330 then retrieves the original data bit sequence. In [73], the authors 331 have derived a soft-output ML detector for recovering the 332 desired signals with the aid of soft decisions, and have shown 333 that the soft-output ML detector outperforms its hard-decision 334 counterpart. Moreover, in [74], the authors have exploited the 335 inherent ML data detection in the context of STSK systems 336 and proposed a semi-blind iterative channel estimation and 337 data detection scheme for STSK, which is capable of reducing 338 the training overhead required. Furthermore, a low-complexity 339 multi-stage ML detector was proposed for the ICGH of [12], 340 which adopts the principles of SM. The proposed detector 341 estimates the APM symbol prior to detecting the TA index. 342 Unlike the ML detector of other spatial multiplexing MIMO 343 techniques, the complexity of the single-stream ML receiver 344 only increases linearly with the number of TAs. However, as

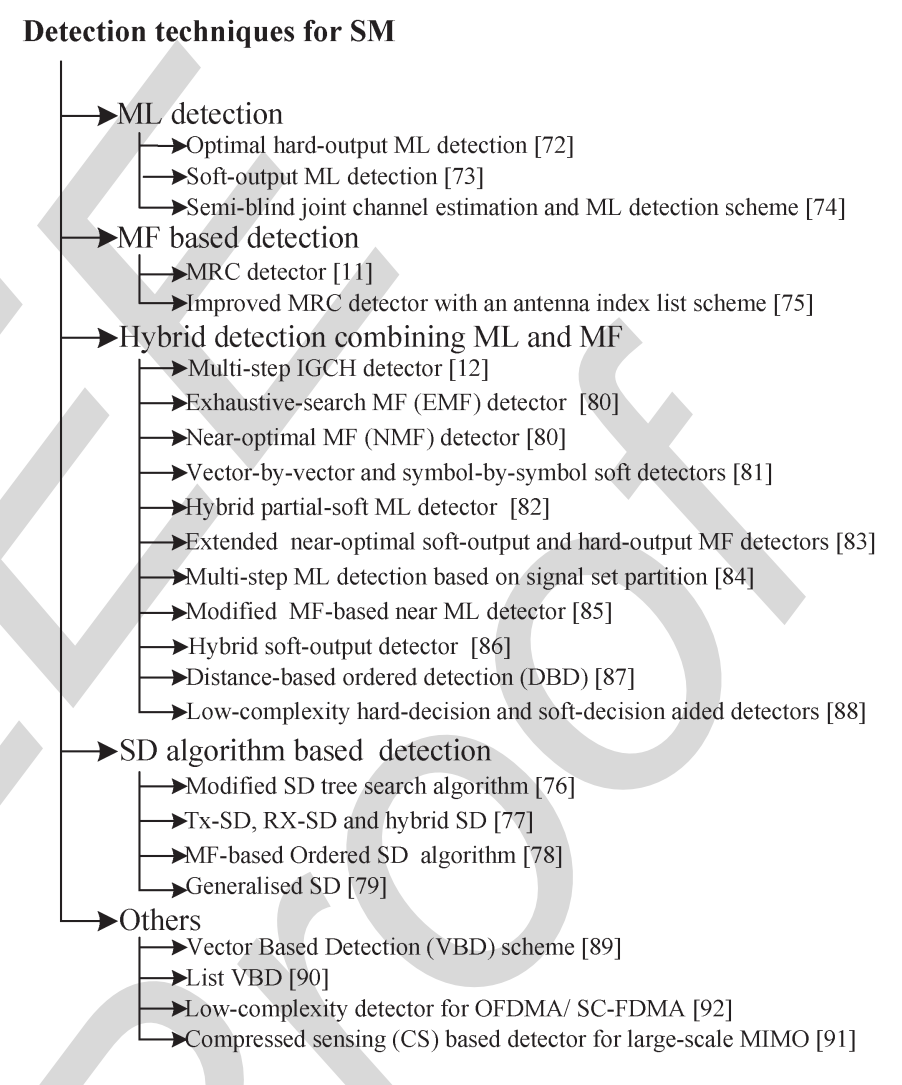

Fig. 4. Overview of SM detectors and related techniques.

the transmission rate increases, even the complexity of the ML 345 single-stream detector might become excessive. 346

Among the promising alternatives, the MF-based detector 347 exhibits a considerably reduced complexity, since the activated 348 TA index and the modulated APM constellation point are sep- 349 arately estimated. However, as mentioned in [11], the conven- 350 tional MF detector, namely the MRC, only performs well under 351 the idealized assumption of perfect channel knowledge. This 352 detector was improved in [75] and a TA index list based scheme 353 was introduced for all the conventional MIMO channels. 354

For the sake of approaching the single-stream ML detector's 355 performance without any substantial performance degradation, 356 beneficial hybrid detectors were designed for the SM family 357 in [80]-[88], which combine the modified MF concept of [11] 358 and the reduced-complexity exhaustive ML search philosophy 359 
360 of [72]. For example, in [80], two modified MF-based detec361 tors, namely the Exhaustive-search based MF (EMF) detector 362 and the Near-optimal MF (NMF) detector were proposed for 363 achieving a better performance than the conventional MF detec364 tor. However, the EMF has to invoke an exhaustive signal space 365 search at the MF's output for maintaining the ML's perfor366 mance, which prevents the detector from achieving a significant 367 reduction in complexity, when high data rates are required. By 368 contrast, the NMF detector further reduced the EMF's complex369 ity, but naturally it performs worse than the ML detector [72]. 370 To overcome this limitation, the authors of [83] proposed an 371 extended NMF detector, which relies on finding multiple high372 probability indices for the sake of attaining further performance 373 improvements. Then, this improved NMF detector was further 374 simplified in [87] and [88]. Considering that SM-MIMO sys375 tems typically rely on powerful channel codes, an attractive 376 detector has to provide soft-decision-based information. In [44] 377 and [73], an optimal Maximum a Posteriori (MAP) detector 378 was invoked for turbo-coded SM schemes. However, it suffers 379 from the problem of having a high complexity. In [81], the au380 thors have proposed a low-complexity vector-by-vector based 381 soft-detector operating on a symbol-by-symbol basis, where the 382 associated complexity was considerably reduced compared to 383 that of the max-log MAP detector's, albeit this was achieved at 384 the cost of a modest performance degradation.

385 On the other hand, the SD [93], [94], which is widely used 386 in spatial multiplexing systems, avoids the exhaustive search 387 of the potentially excessive-complexity signal constellation by 388 examining only those candidate solutions that lie inside an 389 SNR-dependent decoding sphere. However, the conventional 390 SD and the more advanced SD methods [94] are oblivious of the 391 specific principle of SM, namely that only a single TA is active 392 at any given time instant. As a result, the SD methods designed 393 for spatial multiplexing MIMOs cannot be directly applied 394 to SM-MIMO detection. In [76], a modified SD algorithm 395 referred to as SM-SD was proposed, which is based on the tree396 search structure. The SM-SD algorithm exploits the specific 397 transmission mode of SM and hence attains a considerable 398 complexity reduction. However, the performance of the SM399 SD algorithm depends on the particular choice of the SNR400 dependent initial search-radius as well as on the transmitter 401 parameters. Hence, in [78], an Ordered SD (OSD) algorithm 402 was proposed for the family of SM arrangements for the sake 403 of reducing the receiver's complexity, while maintaining the op404 timum single-stream ML performance, which searches through 405 the signal space sequentially according to the sorted TA set. 406 Recently, a generalized version of the SM-SD was proposed in 407 [77] and [79].

408 Relying on a novel approach, in [89] the authors have pro409 posed a new Vector Based Detection (VBD) scheme for SM, 410 which is suitable for high-order APM constellations. In [90], 411 an improved VBD scheme, namely the list-VBD was proposed, 412 where the TA index detection is performed first and a list of the 413 best candidates survives. As indicated in Section I, the family 414 of SM constitutes an attractive framework for the emerging 415 family of large-scale MIMO systems in reducing the hard416 ware costs and detection complexity, which becomes realistic 417 at microwave frequencies. Since ML detection of high-order
APM schemes in large-scale high-rate MIMO systems has 418 a potentially excessive complexity, in [91] a low-complexity 419 Compressed Sensing (CS) based detector was proposed for 420 overcoming this problem by exploiting the sparsity in SM 421 signaling. Again, the family of SM has also been effectively 422 extended to the Orthogonal Frequency Division Multiple Ac- 423 cess (OFDMA)/Single-Carrier Frequency Division Multiple 424 Access (SC-FDMA)-aided architecture and some related low- 425 complexity detectors were proposed in [92].

426

Additionally, most of the above-mentioned detectors assume 427 that perfect CSI is available at the receiver. However, it is chal- 428 lenging to acquire accurate CSI in high-speed vehicles and mul- 429 tiple antenna systems. In order to dispense with CSI-estimation, 430 the class of Differentially-encoded STC (DSTC) was proposed 431 in [95] and [96]. Specifically, the Unitary Space-Time Modula- 432 tion (USTM) scheme does not require CSI estimation and hence 433 facilitates non-coherent detection at the receiver. Motivated by 434 the concept of DSTC, the design of non-coherent SM-MIMO 435 schemes was investigated in [43], [97], and [98]. To be specific, 436 in [43], the differential STSK (DSTSK) concept was proposed 437 with the aid of the Cayley unitary transformation, which has 438 a low-complexity single-stream non-coherent detector. In [97], 439 the DSTSK scheme was further developed for the sake of avoid- 440 ing the nonlinear Cayley transform and a reduced-complexity 441 multiple-symbol differential sphere detector was proposed for 442 rapidly fading channels. Moreover, a PSK-aided differential 443 modulation concept was conceived in [98], which relies on 444 differential decoding while retaining the fundamental benefits 445 of coherent SM-MIMO schemes.

D. Channel Capacity and Error Performance Metric

1) Channel Capacity: The capacity of SM constitutes a vi- 448 tally important research topic. In [12], the authors have derived 449 the capacity of SM in the context of Rayleigh fading chan- 450 nels, assuming continuous-amplitude discrete-time Gaussian 451 distributed transmitted signals. This capacity is also referred to 452 as the Continuous-input Continuous-output Memoryless Chan- 453 nel (CCMC) capacity [7]. However, this assumption cannot be 454 readily satisfied in a practical communication system, unless 455 carefully designed superposition modulation is used [99]. By 456 contrast, in [43] the Discrete-input Continuous-output Memo- 457 ryless Channel (DCMC) capacity [100] of the family of SM 458 scheme was formulated, where the transmitted signals were 459 drawn from finite-alphabet discrete constellations, such as the 460 classic APM schemes [13]. Moreover, a closed-form expression 461 of the mutual information of SM based Multiple-Input Single- 462 Output (MISO) channels was derived and the impact of finite- 463 alphabet inputs on the attainable performance of SM was in- 464 vestigated in [101]. Owing to its particular operating principle, 465 its capacity and the corresponding optimization algorithms still 466 require further research.

467

Fig. 5 shows the CCMC and DCMC capacity curves of the 468 $(4 \times 2)$-element SM-MIMO scheme. Furthermore, the G4- 469 STBC arrangement of [3] was also considered as benchmarkers 470 in Fig. 5. As shown in Fig. 5, the CCMC capacity of the SM 471 scheme is higher than that of G4-STBC. Additionally, observe 472 in Fig. 5 that the DCMC capacity tends to be increased upon 473 


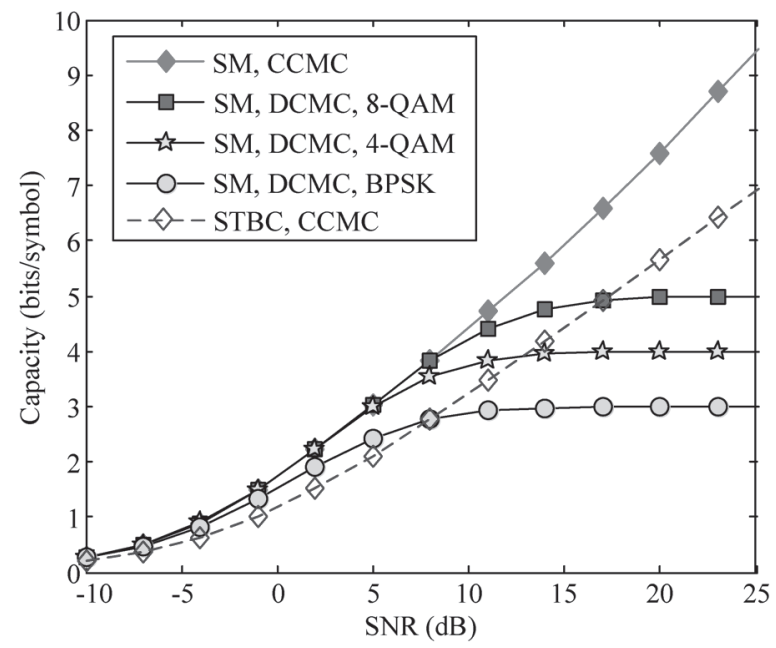

Fig. 5. Bandwidth efficiency of $(4 \times 2)$-element $\mathrm{SM}$ system, comparing the CCMC and the DCMC capacity.

474 increasing the modulation order, as noted in [12]. Moreover, as 475 indicated in [8] and [26], the capacity of SM may be lower than 476 that of the V-BLAST arrangement, however its detection com477 plexity does not depend on the number of transmit antennas. 478 This attractive advantage facilitates the practical application of 479 SM-MIMO.

480 2) Error Performance Metric: The BER performance of SM 481 has also been studied extensively in the context of various 482 channel models and MIMO setups [28]-[34]. Generally, the an483 alytical study of SM-MIMO systems tends to rely on its union 484 bound based approximation [102]. However, apart from the 485 STSK studies of [43] and the investigations of Di Renzo et al. 486 [15], the studies in [27], [28], and [32]-[34] considered the sim487 plified version of SM, namely SSK. For the conventional SM 488 combining SSK with classic APM techniques for the sake of 489 transmitting additional bits, the analytical studies disseminated 490 in [11], [14], [29], and [103] exploited some of the fundamental 491 properties of SM related to the channel's correlation, to its 492 transmit diversity, channel estimation errors and coding gain. 493 For example, in [103] the authors have provided a closed-form 494 Average Bit Error Probability (ABEP) upper bound expression 495 based on the conventional union-bound methods, which also 496 quantified the transmit diversity order of SM. This framework 497 is usually used as a reference for highlighting the advantages 498 of SM over other MIMO arrangements, such as the classic 499 STBC and VBLAST schemes. In [29], an improved union500 bound is formulated, which partitions the ABEP expression of 501 SM-MIMO systems into three terms: the term $P_{\text {spatial }}(\rho)$ only 502 related to the spatial signals (i.e., TA index), the term $P_{\text {signal }}(\rho)$ 503 is only related to the APM signals, while the joint term $P_{\text {joint }}(\rho)$ 504 depends on both the spatial signals and on the APM signals, 505 where $\rho$ is the average SNR. This bound is formulated as

$$
\mathrm{P}_{\mathrm{SM}}(\rho) \leq P_{\text {spatial }}(\rho)+P_{\text {signal }}(\rho)+P_{\text {joint }}(\rho) .
$$

506 Assuming i.i.d. Rayleigh fading channels, $P_{\text {signal }}(\rho)$ pre507 dominantly depends on the minimum ED $d_{\text {min }}$ of the constel508 lation points of APM, while $P_{\text {joint }}(\rho)$ and $P_{\text {spatial }}(\rho)$ mainly 509 depend on the modulus values $\beta_{l}(l=1, \cdots, L)$ of the APM

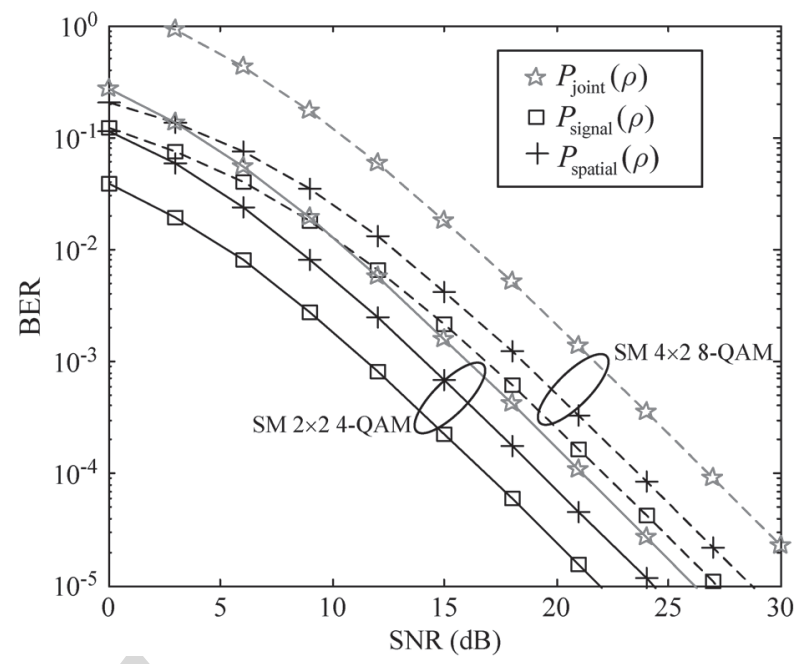

Fig. 6. The ABEPs of SM-MIMO: $P_{\text {signal }}(\rho), P_{\text {joint }}(\rho)$ and $P_{\text {spatial }}(\rho)$.

constellation points, as detailed in [29]. As a result, $\mathrm{P}_{\mathrm{SM}}(\rho) 510$ of (2) depends both on the minimum ED of the specific APM 511 constellations employed, as well as on the absolute values of 512 the APM-symbols. This improved ABEP upper bound of SM 513 provides deeper insights into the interactions of the APM signal 514 constellation and the spatial signal constellation. For example, 515 the interaction term $P_{\text {joint }}(\rho)$ of (2) dominates the performance 516 of SM in diverse popular MIMO configurations, as indicated in 517 Fig. 6. On the other hand, it can also be used for optimizing the 518 system's performance by exploiting any statistical knowledge 519 about the Channel State Information (CSI) at the transmitter 520 and we will discuss in Section III.

Moreover, since the exact ABEP does not have a simple 522 closed form solution, the nearest neighbor approximation was 523 proposed in [104]. Assuming that all the channel inputs are 524 equally likely, the nearest neighbor approximation of the Pair- 525 wise Error Probability (PEP) for a given channel matrix $\mathbf{H}$ can 526 be expressed as [105]

$$
P_{e \mid \mathbf{H}} \approx \lambda \cdot Q\left(\sqrt{\frac{1}{2 N_{0}} d_{\min }^{2}(\mathbf{H})}\right)
$$

where we have $Q(x)=(1 / \sqrt{2 \pi}) \int_{x}^{\infty} e^{-y^{2} / 2} d y$, and $\lambda$ is the 528 number of neighboring constellation points [10] associated with 529 the free distance $(\mathrm{FD}) d_{\min }(\mathbf{H})$ defined as

$$
d_{\min }(\mathbf{H})=\min _{\substack{\mathbf{x}_{i}, \mathbf{x}_{j} \in \mathbb{X}, \mathbf{x}_{i} \neq \mathbf{x}_{j}}}\left\|\mathbf{H P}\left(\mathbf{x}_{i}-\mathbf{x}_{j}\right)\right\|
$$

where $\mathbb{X}$ is the set of legitimate transmit symbols, while $\mathbf{x}_{i} 531$ and $\mathbf{x}_{j}$ are two distinct transmitted symbols in $\mathbb{X}$. In (4), $\mathbf{P}$ is 532 the transmit preprocessing (TPP) matrix, which is the $\left(N_{t} \times 533\right.$ $N_{t}$ )-element identity matrix I for conventional open-loop SM 534 schemes dispensing with TPP.

Note that the nearest neighbor approximation of the PEP will 536 always be slightly lower than that provided by the union bound, 537 since this approximation does not include the errors associated 538 with those legitimate symbols that are farther apart than the 539 FD. However, in case of low SNRs, there is a non-negligible 540 probability of corrupting a symbol into more distant symbols. 541 
542 Nonetheless, the result is quite close to the exact probability of 543 symbol error at high SNRs, as detailed in [105]. Indeed, since 544 the error events mainly arise from the nearest neighbors, the 545 maximization of the FD in (3) directly reduces the probability 546 of error, especially at high SNRs [106]. As a result, the bound 547 of (3) can be adapted for system optimization by exploiting the 548 knowledge of the near-instantaneous CSI, as discussed these in 549 more detail in Section IV.

550 Furthermore, the effects of CSI errors on the achievable per551 formance of SM-MIMOs were further researched in [34] and 552 [107]-[109]. It was found that SM is quite robust to imperfect 553 CSI compared to V-BLAST. For example, in [107] an asymp554 totically tight upper bound on the ABEP was derived for SM 555 under imperfect CSI and the simulation results confirmed that $556 \mathrm{SM}$ is more robust to channel estimation errors than V-BLAST 557 for reasonable practical channel estimation error values.

\section{8 \\ III. APM Constellation Optimization}

559 As indicated in (2), the performance of SM-MIMO systems 560 is highly dependent on the specific APM signal constellation 561 adopted. In a conventional Single-Input Single-Output (SISO) 562 system, the Gray-coded Maximum-minimum distance (MMD) 563 QAM constellation minimizes the Bit Error Ratio (BER) [13]. 564 However, the advantage of MMD-QAM may be eroded in SM565 MIMO systems [29]. This is due to the fact that the BER 566 performance of SM-MIMO systems is jointly determined by 567 the spatial signal (i.e., TA indices), by the classic APM con568 stellation and by their interaction [29]. Hence, a suitable APM 569 scheme has to be designed for this hybrid modulation scheme. 570 Furthermore, SM also allows us to achieve a high transmis571 sion rate by combining its benefits with those of the classic 572 APM schemes, as detailed in [46]-[49]. However, when the 573 source employs higher-order square QAM in order to increase 574 the attainable transmission rate, a high Peak-to-Average-Power 575 Ratio (PAPR) [110] is encountered, hence requiring a low576 efficiency linear power amplifier [111]. To overcome this im577 pediment, peak-power reduction constellation shaping [110] 578 may be employed at the transmitter, albeit this technique im579 poses additional complexity. Thus, for the sake of achieving a 580 high power-efficiency, the choice of the modulation scheme in 581 SM-MIMO systems has to be revisited.

582 The effects of APM schemes on the performance of SM 583 have been investigated in [112]-[114]. More specifically, in 584 [112], the dispersion matrices and the signal constellations were 585 jointly optimized for a near-capacity precoded STSK system, 586 which includes SM as a special case and strikes a flexible rate587 versus-diversity tradeoff. It was also shown in [80] that the star588 QAM aided STSK scheme outperforms its MMD based square589 QAM aided counterpart. This is because the STSK's achievable 590 performance depends both on the minimum ED of the APM 591 constellation employed, as well as on the absolute values of the 592 modulated symbols, which may also be valid for SM systems, 593 as shown in (2) [29]. More recently, in [31] low-complexity, 594 yet single-stream ML transmit diversity schemes have been 595 studied by analyzing the impact of the spatial constellation and 596 shaping filters. In [70], a Hamming code construction technique 597 was proposed as a modulation design strategy for SSK-based

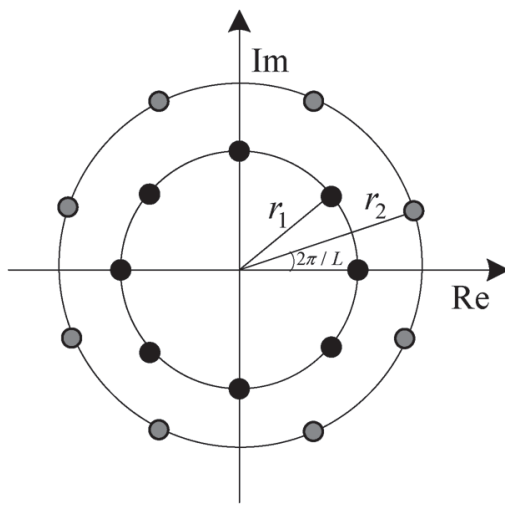

Fig. 7. The complex signal constellation of 16-ary star-QAM. The symbols are evenly distributed on two rings, and the phase differences between the neighboring symbols on the same ring are equal.

systems for the sake of improving their error probability. In 598 [113], a new SM constellation design strategy was proposed 599 based on the ED of the constellation, which retains the key 600 advantages of SM, while activating multiple TAs. In [114], 601 two approaches were investigated with the goal of designing 602 the SSK's transmit constellation space by relying either on 603 the idealized simplifying assumption of having perfect CSI or 604 on the more practical scenario of imperfect CSI at the trans- 605 mitter, in order to increase the distance between each pair of 606 the received combined TA-APM vector. The above-mentioned 607 techniques were however mainly conceived for STSK and SSK 608 schemes, but may not be readily applicable to the conventional 609 SM scheme.

610

In [29], the performance of SM systems relying both on 611 conventional QAM and PSK modulation were studied, demon- 612 strating that in some MIMO setups, the PSK-modulated SM 613 scheme may outperform the identical-throughput MMD-QAM 614 SM scheme. More specifically, as shown in [29] and [115], for 615 certain SM-MIMO configurations, $P_{\text {signal }}(\rho)$ of (2) is signifi- 616 cantly higher than the sum of $P_{\text {joint }}(\rho)$ and $P_{\text {spatial }}(\rho)$, which 617 implies that the minimum ED of APM constellations dominates 618 the performance of SM. In this scenario, MMD-QAM may 619 constitute an attractive APM candidate for minimizing the 620 ABEP. By contrast, as shown in Fig. 6, if $P_{\text {signal }}(\rho)$ is lower 621 than the sum of $P_{\text {joint }}(\rho)$ and $P_{\text {spatial }}(\rho)$, which implies that 622 the moduli of the APM constellation points dominates the 623 $\mathrm{P}_{\mathrm{SM}}(\rho)$ term, then a constant-modulus modulation scheme, 624 such as PSK, may be optimal, as indicated in [29]. Recall that 625 $P_{\text {signal }}(\rho)$ of (2) is dominated by the minimum ED $d_{\text {min }}$, while 626 $P_{\text {joint }}(\rho)$ and $P_{\text {spatial }}(\rho)$ mainly depend on the modulus values 627 $\beta_{l}(l=1, \cdots, L)$ of the APM constellation adopted. Note that 628 the modulus values $\beta_{l}(l=1, \cdots, L)$ are represented by the 629 Frobenius norms of the APM constellation points. These results 630 suggested that for the sake of jointly minimizing $P_{\text {signal }}(\rho), 631$ $P_{\text {joint }}(\rho)$ and $P_{\text {spatial }}(\rho)$ of (4), we can readily focus our 632 attention on design of $d_{\min }$ and on the $\beta_{l}$ parameters of APM. 633

On the other hand, star-QAM [13] constitutes a special case 634 of circular APM, which is capable of outperforming the classic 635 square-shaped QAM constellation in peak-power-limited sys- 636 tems. Hence its diverse relatives have been adopted in most 637 of the recent satellite communication standards, such as the 638 
TABLE II

The Minimum ED of DifFERENT APM SCHEMES

\begin{tabular}{|l|c|c|c|c|c|}
\hline Modulation order $(L)$ & 2 & 4 & 8 & 16 & 32 \\
\hline PSK & $d_{\min }=2$ & $d_{\min }=\sqrt{2}$ & $d_{\min }=0.76$ & $d_{\min }=0.39$ & $d_{\min }=0.19$ \\
\hline QAM & -- & $d_{\min }=\sqrt{2}$ & $d_{\min }=0.81$ & $d_{\min }=0.63$ & $d_{\min }=0.41$ \\
\hline Star-QAM & $d_{\min }=2$ & $d_{\min }=\sqrt{2}$ & $d_{\min }=0.91$ & $d_{\min }=0.57$ & $d_{\min }=0.40$ \\
\hline
\end{tabular}

639 Digital Video Broadcast System (DVB) S2, DVB-SH, as well 640 as in the Internet Protocol over Satellite (IPOS) and Advanced 641 Broadcasting System via Satellite (ABS-S) [116]. To elaborate 642 a little further, the star-QAM constellation is composed of 643 multiple concentric circles and it was shown to be beneficial in 644 the context of STSK systems [80]. However, the constellations' 645 optimization has not been carried out for star-QAM aided SM. 646 In order to make the choice of the APM parameters $d_{\text {min }}$ 647 and $\beta_{l}$ as flexible as possible, we consider a class of star648 QAM constellations, which subsumes the classic PSK as a 649 special case, but may also be configured for maximizing the 650 minimum ED of the constellation by appropriately adjusting 651 the ring ratios of the amplitude levels. For the sake of sim652 plicity, we consider the example of a twin-ring 16-star-QAM 653 constellation having a ring-ratio of $\alpha=r_{2} / r_{1}$ as shown in 654 Fig. 7. The symbols are evenly distributed on the two rings 655 and the phase differences between the neighboring symbols 656 on the same ring are equal. Unlike the conventional twin-ring 657 star-QAM constellation [116], the constellation points on the 658 outer circle of star-QAM constellation are rotated by $2 \pi / L$ 659 degrees compared to the corresponding constellation points on 660 the inner circle. Hence again, the conventional PSK constitutes 661 an integral part of our star-QAM scheme, which is associated 662 with a ring-ratio of $\alpha=1$. Note that although this twin-ring 663 star-QAM constellation has indeed been invoked for nonco664 herent detection [117], it has not been considered whether 665 this constellation can be directly applied to SM for achieving 666 performance improvements.

667 Table II summarizes the minimum EDs $d_{\min }$ between the 668 constellation points for different APM schemes, where the 669 modulation order is the number of the constellation points. 670 Moreover, the $L$-PSK $/ L$-QAM schemes in [13] are used. It is 671 shown that the star-QAM is capable of achieving almost the 672 same minimum ED as the MMD-based QAM [8].

673 Given an $\left(N_{r} \times N_{t}\right)$-element MIMO setup having a trans674 mission rate of $m_{\text {all }}$, and $L$ modulation levels, the goal of star675 QAM aided signaling constellation optimization is to find the 676 ring-ratio $\alpha$, which minimizes the ABEP of SM-MIMO of (2). 677 Following this approach, the related optimization problem may 678 be formulated as

$$
\left\{\begin{array}{c}
\alpha^{*}=\min _{\alpha} \mathrm{P}_{\mathrm{SM}}(\rho) \\
\text { s.t. } \alpha \geq 1
\end{array}\right.
$$

679 Based on an exhaustive numerical search, for example, for 680 the 16-star-QAM aided $(4 \times 4)$-element SM-MIMO, the opti$681 \mathrm{mal}$ ring ratio was found to be $\alpha^{*}=1.7$ [118]. According to (2), 682 this optimized star-QAM aided SM scheme provides an SNR 683 gain of about $3 \mathrm{~dB}$ over the conventional 16-PSK modulated 684 SM scheme and an SNR gain of about $1.1 \mathrm{~dB}$ over the identical685 throughput Gray-coded MMD 16-QAM modulated SM scheme 686 at $\mathrm{BER}=10^{-5}$. Note that the optimized star-QAM constella-

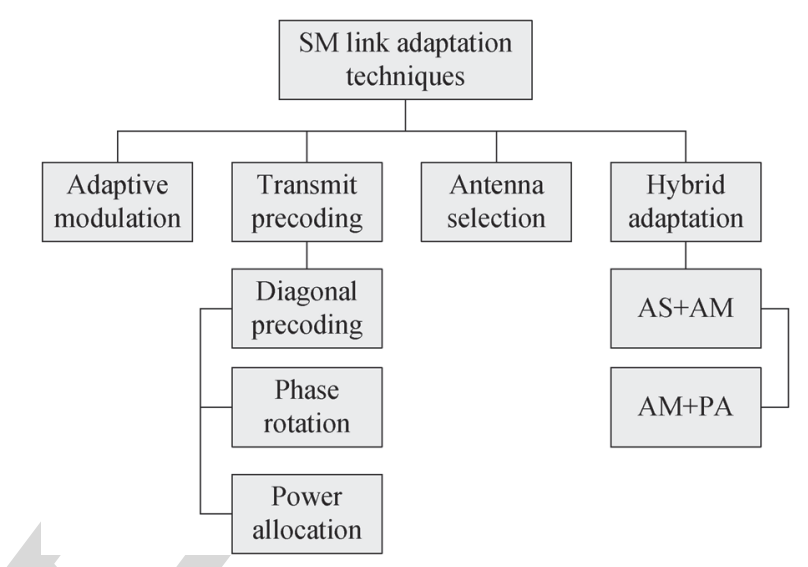

Fig. 8. Classification of the LA techniques designed for SM-MIMO. Here, AS+AM: antenna selection combined with adaptive modulation, AM+PA: adaptive modulation combined with power allocation.

tion can be designed off-line based on the CSI statistics (i.e., 687 the fading type) for different SM-MIMO systems and hence 688 the resultant system does not need any feedback. Next, we will 689 introduce a suite of beneficial adaptation techniques based on 690 the assumption that the knowledge of the near-instantaneous 691 channel matrix is available at the receiver in the frequency flat- 692 fading channel.

\section{Link ADAPTATION TECHNiQUES}

Link Adaptation (LA) has an important role in wireless 695 communication systems [39]-[41]. Traditionally, LA refers to 696 the concept of dynamically adjusting the transmit parameters, 697 such as the modulation order and coding rate according to the 698 near-instantaneous channel conditions. LA has been extensively 699 studied in the conventional MIMO context for the sake of im- 700 proving the achievable multiplexing and diversity performance. 701 However, it has not been considered, whether these existing LA 702 techniques can be directly applied to SM-based transmission 703 systems. Note that the introduction of LA techniques in SM- 704 MIMO should not jeopardize the advantages of SM, such as the 705 avoidance of the IAI, IAS and multiple RF chains [11]. This 706 makes the design of LA algorithms more challenging. In order 707 to increase the robustness of the SM-MIMO system, several 708 limited-feedback aided LA techniques have been proposed in 709 [30], [104], [115], and [119]-[130], as summarized in Fig. 8. 710 Depending on the MIMO scheme's degree freedom, these 711 techniques can be roughly divided into four types, namely into 712 Adaptive Modulation (AM) [104], [115], [119], [120], transmit 713 precoding (TPC) [30], [103], [121]-[125], Antenna Selection 714 (AS) [126]-[128] and Hybrid Adaptation (HA) techniques re- 715 lying on diverse combinations of the above three [115], [129], 716 [130], as shown in Fig. 8. To elaborate a little further, the 717 


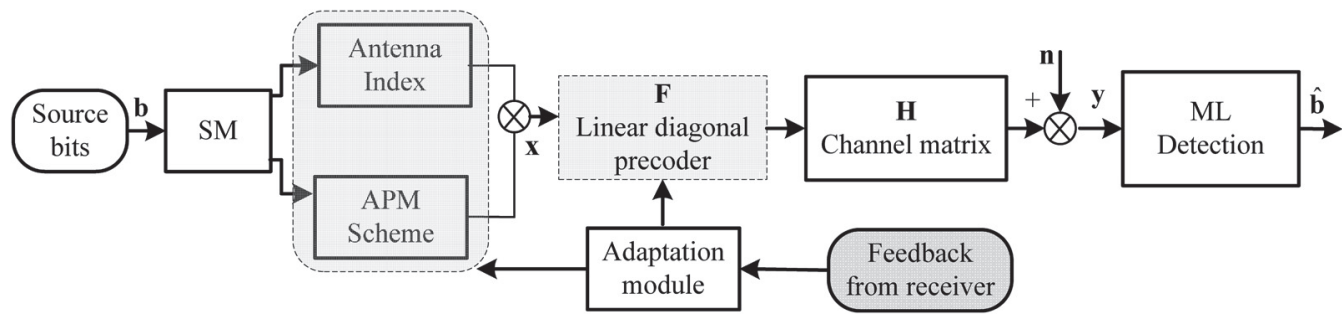

Fig. 9. Block diagram of LA-aided MIMO communication systems.

718 general philosophy of a LA-aided SM-MIMO system obeying 719 the architecture of Fig. 9 can be summarized as follows.

720 Algorithm 2: The adaptation process of LA-aided SM721 MIMO systems

1) Consider an $\left(N_{r} \times N_{t}\right)$-element SM-MIMO system associated with the transmission rate $m_{\text {all }}$;

2) The receiver estimates the CSI and decides upon the optimum transmit mode, which is then sent back to the transmitter through a low-rate feedback channel;

3) The transmitter processes the feedback information and employs the optimum transmission mode (i.e., the modulation orders and the precoding matrix) for its transmission.

731 Having formulated the SM-MIMO's LA algorithm, let us 732 now describe the class of LA techniques with the aid of 733 Fig. 8 developed for the family of SM-MIMO schemes in more 734 detail below. Note that in this treatise only the TPC matrix $735 \mathbf{P}$ and the transmit symbol $\mathbf{x}$ are adapted in response to the 736 near-instantaneous channel conditions in order to improve the 737 system's performance, as indicated in (4).

\section{A. Adaptive Modulation}

739 Again, AM techniques are capable of alleviating the adverse 740 effects of channel fading, so as to achieve an increased data 741 rate or a reduced BER [131], which have hence been adopted 742 in most of the recent communication standards, such as 3GPP, 743 3GPP2, IEEE 802.11a, IEEE 802.15.3 and IEEE 802.16 [132]. 744 SM may also be beneficially combined with AM for adjust745 ing the transmission parameters for the sake of accommodating 746 time-varying channels. Therefore, the beneficial combination of $747 \mathrm{AM}$ and SM-MIMO techniques is a promising design alterna748 tive for high-rate wireless systems.

749 To this end, adaptive SM-MIMO architectures relying on 750 different combinations of modulation/coding schemes were 751 proposed in [120], which aimed for maximizing the channel 752 capacity at a predefined target BER, rather than for optimizing 753 the BER. By contrast, in [104] a near-instantaneously Adaptive 754 SM (ASM) scheme was proposed for improving the attainable 755 system performance, while maintaining a fixed average transmit 756 rate with the aid of AM techniques. In ASM, the receiver 757 requests the most suitable modulation order to be used by 758 the transmitter for each TA and/or time-slot. Assuming that 759 no-transmission, BPSK and $M$-QAM are available for each
TA, which are represented by the set $\mathbb{M}_{\text {all }}$, the detailed design 760 procedure of ASM schemes can be summarized as follows: 761

Algorithm 3: Adaptive SM

1) Given the transmit parameters as: $N_{t}, N_{r}$ and the trans- 763 mission rate $m_{\text {all }}$, generate all the legitimate modulation 764 order combinations for a given $m_{\text {all }}$ and represent these 765 combinations as a set $\mathbf{R}=\left\{\mathbf{r}_{1}, \mathbf{r}_{2}, \cdots, \mathbf{r}_{j}, \cdots, \mathbf{r}_{J}\right\}, 766$ where we have $\mathbf{r}_{j}=\left[r_{j}^{1}, \cdots, r_{j}^{n} \cdots, r_{j}^{N_{t}}\right]$ and $r_{j}^{n}$ denotes 767 the modulation order for the $n$th $\left(n=1,2, \cdots N_{t}\right)$ TA of 768 the $j$ th ASM combination.

769

2) Based on the optimization rule, such as the nearest neigh- 770 bor approximation of (3), we can achieve a performance 771 gain by maximizing $d_{\min }(\mathbf{H})$ with the aid of switching 772 among these candidates.

773

3) Then, the corresponding index of the optimal ASM mode 774 is fed back to the transmitter, which transmits the symbols 775 accordingly.

776

In (3), the conditioned PEP is a monotonically decreasing 777 function of $d_{\min }(\mathbf{H})$. Hence, the attainable system performance 778 can be improved by maximizing the FD $d_{\min }(\mathbf{H})$ by adapting 779 the transmit parameters. As an example, let us consider a 780 $(2 \times 2)$-element SM-MIMO transmission scheme associated 781 with $m_{\text {all }}=3$ bits/symbol under a channel realization matrix 782 $\mathbf{H}$, which is given by

$$
\mathbf{H}=\left[\begin{array}{cc}
0.26-0.75 \mathrm{i} & 1.33+0.49 \mathrm{i} \\
0.03+1.30 \mathrm{i} & -0.61+0.25 \mathrm{i}
\end{array}\right]
$$

Let us assume that no-transmission, BPSK, QPSK, 8-QAM, 784 16-QAM, 32-QAM and 64-QAM are available for each TA and 785 these schemes are represented as $\mathbb{M}_{\text {all }}=\{0,2,4,8,16,32,64\}$, 786 where the no-transmission mode has the identifier of $M=0,787$ while the BPSK and QPSK constellations are denoted as $M=2788$ and $M=4$ respectively. For $m_{\text {all }}=3$ bits/symbol, we have five 789 ASM mode candidates denoted as $\mathbf{R}=\left\{\mathbf{r}_{1}, \mathbf{r}_{2}, \mathbf{r}_{3}, \mathbf{r}_{4}, \mathbf{r}_{5}\right\}=790$ $\{[16,0],[2,8],[4,4],[8,2],[0,16]\}$, where $\mathbf{r}_{1}=[16,0]$ repre- 791 sents that 16-QAM and no-transmission are assigned to the 792 first and the second TA, respectively, while the candidate [4,4] 793 corresponds to the conventional non-adaptive SM scheme using 794 QPSK for both TAs.

Based on Algorithm 3, Fig. 10 shows the detailed actions of 796 the ASM scheme for this 3-bits/channel-use system. As shown 797 in Fig. 10, the five ASM modes (the legitimate modulation 798 


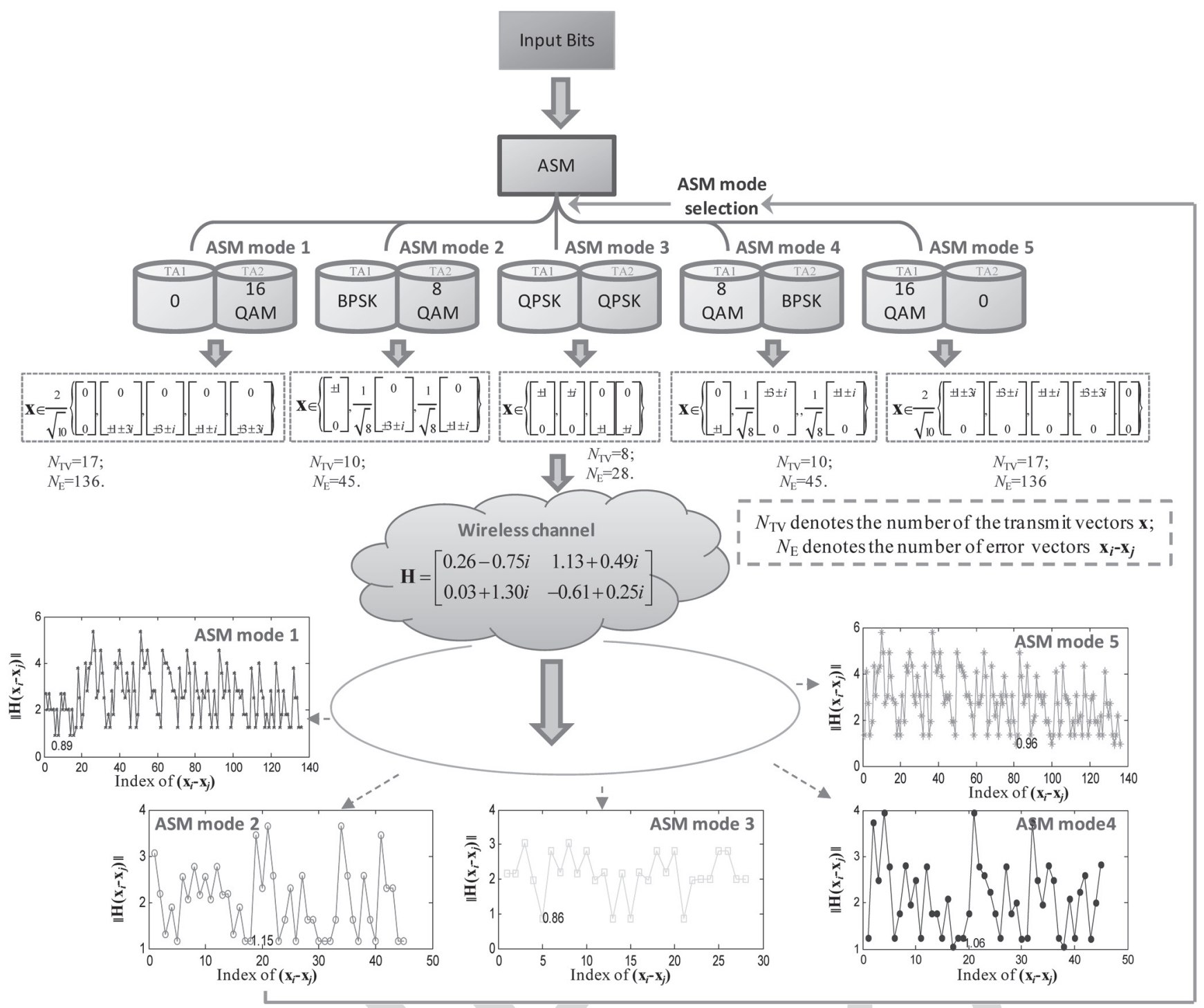

Fig. 10. The example of ASM associated with $(2 \times 2)$-element MIMO channels at a throughput of $m_{\text {all }}=3$ bits/symbol.

799 order combinations) are generated first. For each ASM mode, 800 we can calculate its legitimate transmit symbols $\mathrm{x}$ and its 801 corresponding error vectors. For example, as shown in Fig. 10, 802 the number of $\mathrm{x}$ combinations is $N_{\mathrm{TV}}=8$ for the ASM mode 3 803 (the candidate $[4,4]$ ), while the corresponding number of the 804 error vectors $\mathbf{e}_{i j}=\mathbf{x}_{i}-\mathbf{x}_{j}, i \neq j$ of (4) is $N_{E}=\left(\begin{array}{c}2 \\ N_{\mathrm{TV}}\end{array}\right)=28$. 805 Here, each error vector $\mathbf{e}_{i j}$ is given a specific index, which 806 is associated with its corresponding distance $\left\|\mathbf{H e}_{i j}\right\|$. Then, 807 the minimum value of $\left\|\mathbf{H e}_{i j}\right\|$ among all the legitimate error 808 vectors is found, which determines the FD of this ASM mode. 809 In Fig. 10, the FD of the ASM mode 3 is 0.86. For other ASM 810 modes, we can use the same method of determining the corre811 sponding FDs. Observe in Fig. 10 that ASM mode 2 has the 812 highest FD for the ASM candidate of [2,8]. The corresponding 813 ASM mode index 2 is then fed back to the transmitter.

814 As indicated above, the Modulation Order Selection (MOS) 815 of ASM turns out to be a demanding process, because the 816 global optimum is found by carrying out an exhaustive search 817 across the entire ASM's mode-candidate set. For example, for 818 an ASM scheme associated with $N_{\mathrm{t}}=8$ and 4 bits/symbol 819 transmission, we need a global search of 154,645 candidates, which results in an excessive complexity and feedback load, 820 when high data rates are required. To circumvent this problem, 821 the probabilities of occurrence for the ASM candidates were 822 evaluated theoretically in [119]. More specifically, all legiti- 823 mate ASM-mode candidates were classified according to their 824 variances and FD. It was shown that for most of the practical 825 channel realizations the probability that the maximum FD oc- 826 curs when all the TAs have the same modulation order is high. 827 As a result, only the specific ASM mode candidates associated 828 with lower variances were earmarked for the optimization in 829 Algorithm 3. Based on this result, a One Bit Re-Allocation 830 (OBRA) algorithm was proposed in [119] for the ASM mode 831 selection. OBRA-ASM imposes both a lower complexity and 832 a lower feedback requirement than that of the ASM relying 833 on a potentially excessive-complexity exhaustive search, while 834 imposing a marginal performance degradation. ${ }^{1}$

\footnotetext{
${ }^{1}$ Note that ASM may transmit an unequal number of bits in different time slot. Hence, this mismatch in the transmission frame-length will result in a potential error propagation effect at the detector, which may be mitigated using channel coding techniques, as detailed in [69].
} 


\section{B. Transmit Precoding Techniques}

837 Similar to the AM technique, Transmit Precoding (TPC) is 838 another attractive LA regime, which exploits the knowledge of 839 the CSI at the transmitter, in order to match the transmission 840 parameters to the instantaneous channel conditions. A bene841 ficial solution to this problem is to use the TPC matrix $\mathbf{P}$ 842 of (4) for enhancing the attainable performance. There is a 843 paucity of literature on how to design both linear and non-linear 844 precoders for conventional MIMO schemes [39]. To be specific, 845 non-linear precoding may be more powerful than its linear 846 counterparts, but linear TPC usually achieves a reasonable per847 formance at a significantly lower complexity. Moreover, most 848 of the precoders were designed using a capacity-maximization 849 approach [39], although in practice minimizing the BER may 850 be more important, than maximizing the mutual information or 851 the capacity [40].

852 1) Diagonal Precoding: The SM technique employed in 853 conjunction with a precoding scheme, where the transmitted 854 symbols are appropriately weighted according to the near855 instantaneous channel condition constitutes an attractive so856 lution in terms of improving the system's BER performance. 857 One of the key design challenges of the precoded SM-MIMO 858 architectures is to construct a beneficial precoding matrix $\mathbf{P}$ 859 that relies on a modest amount of feedback information, while 860 retaining all the single-RF benefits of SM-MIMOs.

861 To this end, in [103] a beamforming codebook was designed 862 for optimizing the coding gain of SM-MIMO in the presence 863 of spatial correlation amongst the fading envelopes of the TAs. 864 Recently, a closed-loop TPC method was invoked for providing 865 both diversity and coding gains in the context of GSSK [124], 866 which activated more than one TAs for transmission. However, 867 the above-mentioned schemes considered only a special case 868 of SM, namely SSK. As a result, the schemes proposed for 869 SSK may not be directly applicable to the conventional SM 870 scheme. By contrast, in [133] a TPC technique was used for 871 improving the signal design for a new class of SM, namely 872 for Receiver-SM (R-SM). Moreover, in [100] the authors in873 vestigated the effects of finite-alphabet inputs on the achievable 874 capacity of SM for transmission over MISO channels and 875 then developed a TPC scheme for improving this performance 876 metric.

877 In this section, we continue by considering a novel TPC 878 scheme based on maximizing the FD for the family of SM879 MIMO systems. Note that since the attainable performance of 880 the optimum single-stream ML receiver depends on the FD 881 of the received signal constellation [29], the maximization of 882 the FD directly reduces the probability of error. In order to 883 retain all the single-RF related benefits of SM, we designed 884 the TPC matrix $\mathbf{P}$ to be a diagonal matrix formulated as $885 \mathbf{P}=\operatorname{diag}\left\{p_{1}, \cdots, p_{n}, \cdots, p_{N_{t}}\right\}$. Note that although there are 886 various diagonal matrix aided TPCs proposed for the family 887 of conventional MIMO schemes, they tends to aim for diag888 onalizing the channel matrix [39], which may jeopardize the 889 advantages of SM-MIMOs. As a result, the conventional TPC 890 techniques proposed for classic MIMO schemes, such as the 891 STBC and VBLAST, may not be directly suitable for the family 892 of SM-MIMOs.
In order to identify the specific TPC parameters $p_{n}(n=893$ $1, \cdots, N_{t}$ ), which are capable of maximizing the FD, we have 894 to determine all the $N_{t}$ parameters $p_{n}\left(n=1, \cdots, N_{t}\right)$. Since 895 it may become excessively complex to jointly optimize these 896 $N_{t}$ parameters in the complex-valued field, we decomposed $\mathbf{P} 897$ as $\mathbf{P}=\overline{\mathbf{P}} \boldsymbol{\Theta}=\operatorname{diag}\left\{\bar{p}_{1} e^{j \theta_{1}}, \cdots, \bar{p}_{n} e^{j \theta_{n}}, \cdots, \bar{p}_{N_{t}} e^{j \theta_{N_{t}}}\right\}$. Be- 898 cause the FD of this particular TA-pair predominantly deter- 899 mines the achievable performance, only the specific TA pair 900 $(g, k)$ associated with the FD is considered and the TPC param- 901 eters are selected for appropriately weighting the SM symbols. 902 As a result, there are only two parameters, namely $p_{g}$ and $p_{k}, 903$ to be searched for. Finding the optimal values of $p_{g}$ and $p_{k}$ as 904 a function of both $\mathbf{H}$ and of the optimal transmit parameters 905 involves an exhaustive search over the vast design-space of 906 $\bar{p}_{g}, \bar{p}_{k}, \theta_{g}$ and $\theta_{k}$, which is overly complex. By considering 907 the power constraint, we have $\bar{p}_{k}=\sqrt{2-\bar{p}_{g}^{2}}$. Moreover, since 908 the phase rotation of the symbol is only carried by two TAs, 909 we can simplify the computation by fixing $\theta_{k}=0$ and then 910 finding the optimal $\theta_{g}$. The proposed low-complexity TPC 911 design algorithm is summarized as follows. 912

Algorithm 4: A low-complexity TPC design algorithm for 913 SM-MIMO

914

1) Given the transmit parameters $N_{t}, N_{r}$ and the transmis- 915 sion rate $m_{\text {all }}$ as well as the channel matrix $\mathbf{H}$, the indices 916 of the TA pair $(g, k)$ associated with the FD of (4) are 917 first obtained. In order to offer an increased FD, the TPC 918 parameters of this TA pair can be dynamically adapted. ${ }^{2} 919$

2) Generate all the legitimate diagonal TPC matrix candi- 920 dates represented as $\mathbf{P}_{\text {cand }}=\operatorname{diag}\left\{1, \cdots, \bar{p}_{g} e^{j \theta_{g}}, \cdots, 921\right.$ $\left.\sqrt{2-\bar{p}_{g}^{2}}, \cdots, 1\right\}$, where we have $\bar{p}_{g}=\sqrt{2} / L_{1} * l_{1}, l_{1}=922$ $0, \cdots, L_{1}$ and $\theta_{g}=2 \pi / L_{2} * l_{2}, l_{2}=0, \cdots, L_{2}$. Here, 923 $L_{1}$ and $L_{2}$ are the quantized parameters, which can 924 be flexibly selected according to the prevalent BER 925 requirements.

3) Based on the above-mentioned optimization rule, we 927 can achieve a performance gain by maximizing the FD 928 $d_{\min }(\mathbf{H})$ by switching among these TPC candidates. Note 929 that the FD of the TPC matrixes $\mathbf{P}_{\text {cand }}$ generated will be 930 compared to that of the conventional scheme and then we 931 select the one having the largest FD as our final result. 932

4) Then, the index of the optimized TPC matrix has to be 933 fed back to the transmitter.

934

Unlike in the traditional TPC method of [39], our proposed 935 scheme is suitable for scenarios relying bandwidth-limited 936 feedback channels, because the TPC design is reduced to the 937 design of a diagonal matrix. Moreover, as demonstrated in 938 Algorithm 4 as few as two elements of the diagonal TPC matrix 939 have to be fed back to the transmitter, regardless of the value 940 of $N_{t}$.

\footnotetext{
${ }^{2}$ Note that if the value of $g$ is the same as $k$, we have to adapt the TPC parameters of the pair $(g, u)$, where the TA $u$ has the maximum channel gain $\left\|\mathbf{h}_{u}\right\|_{F}$. Here, $\mathbf{h}_{u}$ is the uth column of $\mathbf{H}$ and $|\cdot|$ stands for the Frobenius norm.
} 


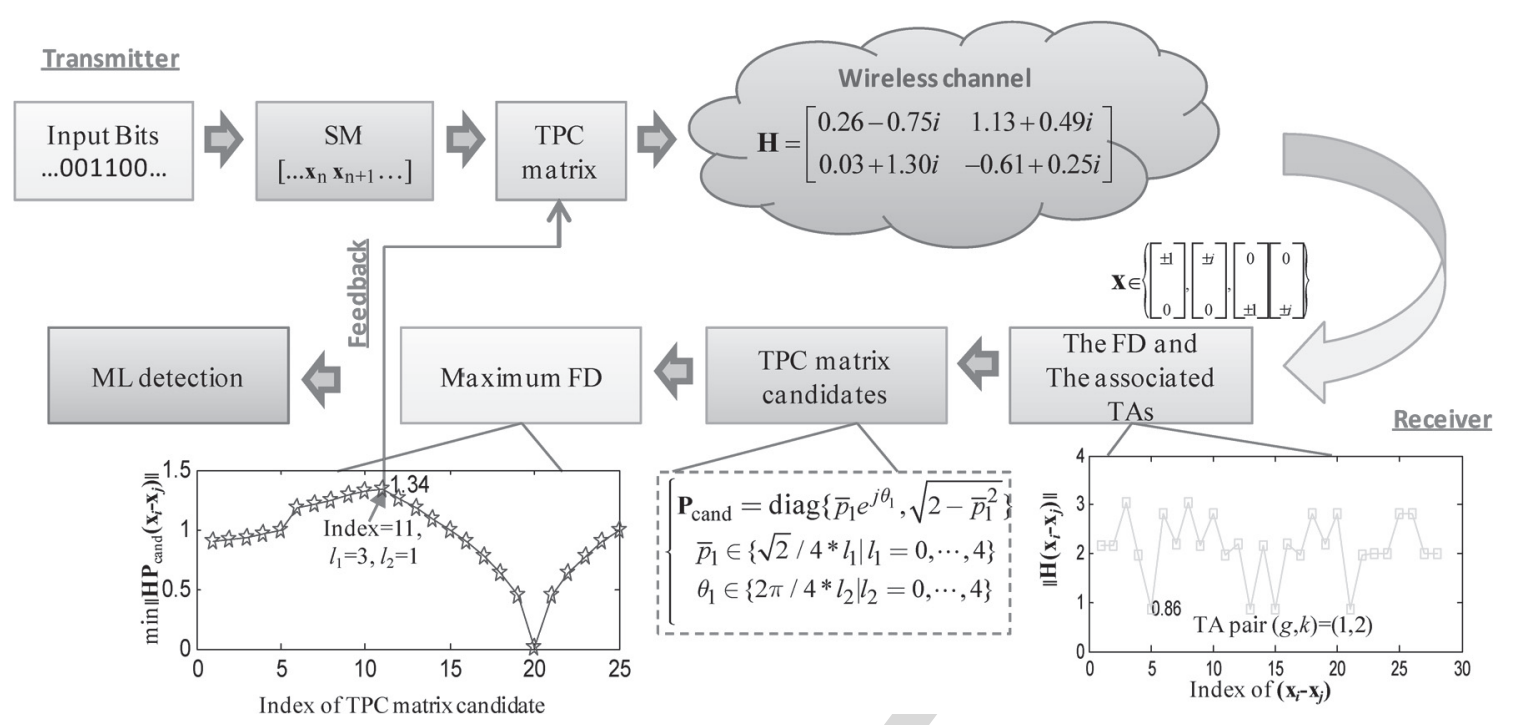

Fig. 11. The example of TPC aided SM.

942 More specifically, revisiting the previous example in 943 Algorithm 3, as shown in Fig. 11, for the same channel 944 realization $\mathbf{H}$, if the TPC matrix $\mathbf{P}$ of Algorithm 4 is used 945 for optimizing the system's performance, where the specific 946 TA-pair $(1,2)$ associated with the FD of 0.86 is first found by 947 using (4), which corresponds to the conventional SM scheme 948 (the ASM mode 3 in Fig. 10). This result implies that the FD 949 is computed for different TAs and the FD of this particular 950 TA-pair predominantly determines the achievable performance. 951 To improve the system's performance, the TPC parameters of 952 this pair should be optimized. Here, the optimized TPC matrix 953 is selected from the quantized TPC matrix set, as shown in 954 Fig. 11, where the quantized parameters $L_{1}$ and $L_{2}$ are se955 lected as $L_{1}=L_{2}=4$. Hence, the number of TPC candidates 956 is $\left(L_{1}+1\right) \times\left(L_{2}+1\right)=25$. We can assign a specific index 957 for each candidate and then calculate its corresponding FD 958 according to (4). As shown in Fig. 11, the specific candidate 959 associated with $l_{1}=3$ and $l_{2}=1$ has the highest FD of 1.34 960 among all the legitimate TPC matrix candidates. Note that if 961 the highest FD of all the legitimate TPC matrix candidates 962 is lower than that of the conventional SM. Based on step 3) 963 of Algorithm 4, The optimal TPC matrix is $\mathbf{P}=\mathbf{I}_{N_{t}}$. The 964 corresponding index of this candidate is then fed back to the 965 transmitter, which appropriately weights the SM modulated 966 symbol.

967 2) Phase Rotation Precoding and Power Allocation: Since 968 the proposed precoder $\mathbf{P}$ consists of two different diagonal 969 matrices $\overline{\mathbf{P}}$ and $\boldsymbol{\Theta}$, we may reduce the complexity of the 970 precoding process in Algorithm $\mathbf{4}$ by employing only a subset 971 of matrices at a modest performance loss. Firstly, when only the 972 diagonal matrix $\Theta$ is considered, this solution may be referred 973 to as the Phase Rotation Precoding (PRP) technique [134], 974 which is usually used for improving the BER, when spatial 975 correlation exists between the TAs of the ML-detection aided 976 V-BLAST architecture.

977 An alternative complexity reduction is achieved by consid978 ering only the diagonal matrix $\overline{\mathbf{P}}$, which can be viewed as 979 a simple form of Power Allocation (PA) [30], [121]-[123].
This arrangement has been intensively researched in the con- 980 text of spatial multiplexing systems [30]. However, these PA 981 approaches designed for spatial multiplexing based MIMO 982 systems may not be directly suitable for the family of SM- 983 MIMO systems, because only a single TA is active in each time 984 slot and hence the PA between the TAs should be carefully con- 985 sidered. In [30], an opportunistic power allocation scheme was 986 conceived for achieving a beneficial transmit diversity gain in 987 SSK-aided MIMO systems relying on two TAs. Then, this 988 feedback-aided PA scheme was further developed in [121]. 989 However, no APM scheme was considered in the above- 990 mentioned PA-aided SSK-MIMO systems and hence their 991 throughput may remain limited. In order to realize the full po- 992 tential of PA techniques in a SM-MIMO context, Algorithm 4993 can also be invoked by simply changing the legitimate diagonal 994 TPC matrix to the PA matrix.

Still considering the example given in Fig. 11, if the PA 996 technique is considered, we gradually assign the appropriate 997 portion of power to each TA of the TA pair $(1,2)$, where the 998 number of PA matrix candidates is $L_{1}+1=5$, as shown in 999 Fig. 12(a). Similar to Fig. 11, we can also assign a specific 1000 index for each candidate and then calculate its corresponding 1001 FD according to (4). As shown in Fig. 12(a), the PA matrix 1002 candidate associated with $l_{1}=3$ has the highest FD of 1.261003 among all the legitimate PA matrix candidates. On the other 1004 hand, as shown in Fig. 12(b), if the PRP technique is invoked, 1005 only the phases of the TA pair $(1,2)$ are adjusted, where the 1006 number of PRP matrix candidates is $L_{2}+1=5$. We observe 1007 from the results of Fig. 12(b) that the PRP matrix candidate 1008 associated with $l_{2}=3$ has the highest FD of 1.3 among all the 1009 legitimate PRP matrix candidates. The index of the optimized 1010 matrix is fed back to the transmitter for allowing the transmitter 1011 to compensate for the effects of channel fading.

1012

3) Performance Results: In Fig. 13, we compared the var- 1013 ious LA-aided SM schemes to the conventional non-adaptive 1014 SM scheme in the context of $(2 \times 2)$-element MIMO chan- 1015 nels at a throughput of $m_{\text {all }}=3$ bits/symbol for transmission 1016 over independent Rayleigh block-flat channels. In all cases we 1017 


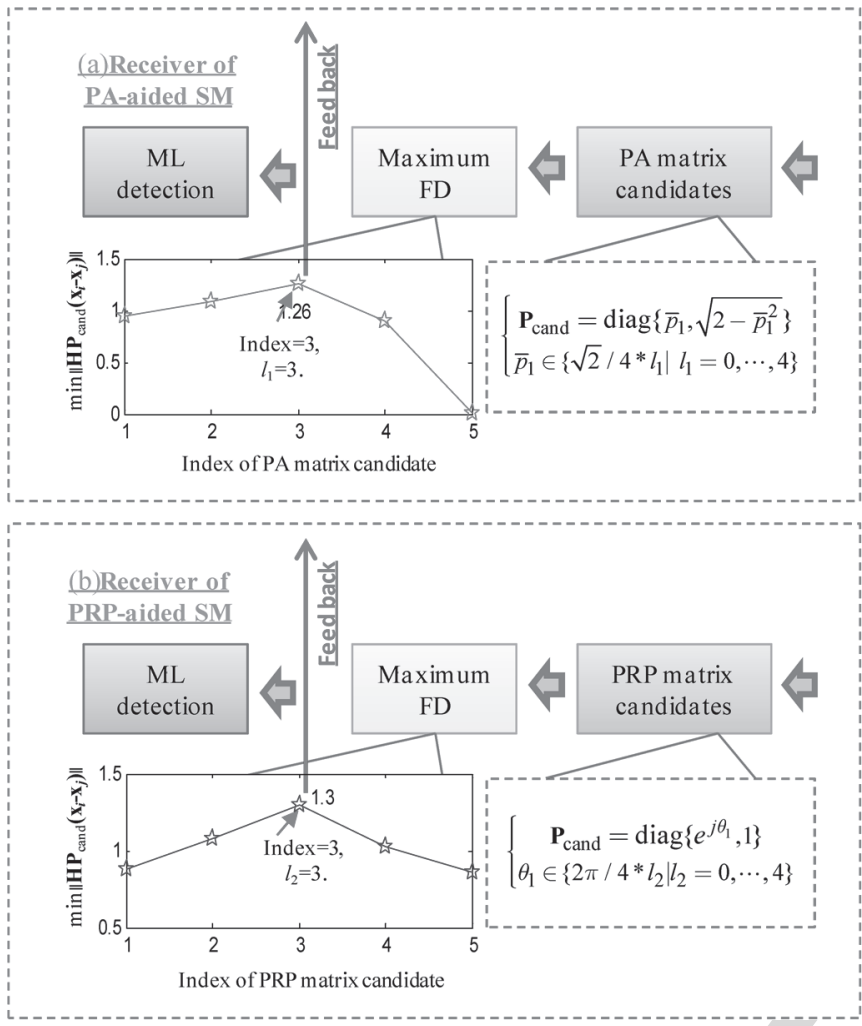

Fig. 12. The example of PA and PRP aided SM system.

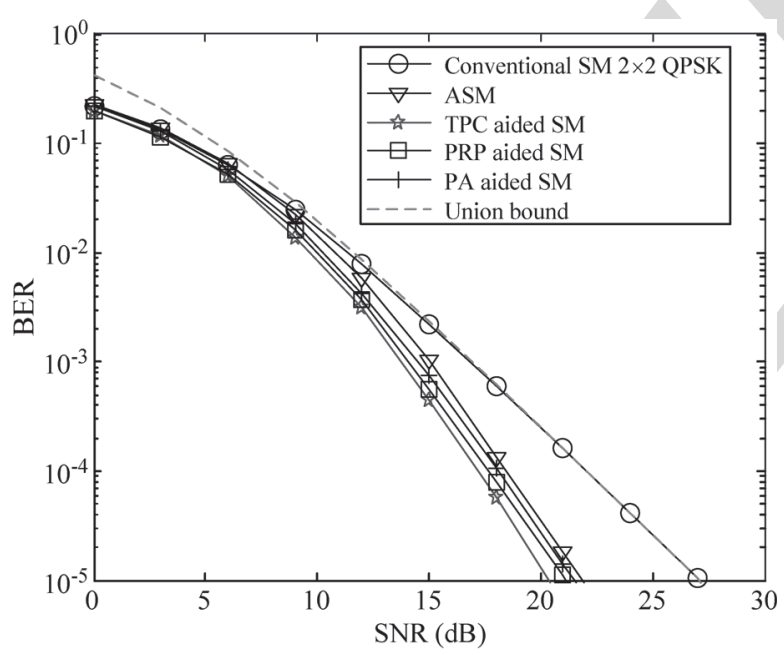

Fig. 13. BER performance of the conventional SM and the LA-aided SM schemes in $(2 \times 2)$-element MIMO channels at a throughput of $m_{\text {all }}=$ 3 bits/symbol.

1018 assumed that the feedback channel is free of errors and delay. ${ }^{3}$ 1019 For completeness, we also added the theoretical upper bound 1020 curve derived with the aid of the union bound [29], [103] of 1021 the conventional SM scheme. Moreover, in the TPC design of 1022 Algorithm 4, we selected $L_{1}=L_{2}=4$.

\footnotetext{
${ }^{3}$ The error-free feedback channel assumption in SM-based schemes may be justifiable, since the feedback channel is usually protected using powerful error correction coding and hence has a low error probability [4]. The effect of imperfect feedback channels in closed-loop MIMO systems has been documented, for example in [135].
}

As expected, the proposed LA-aided schemes beneficially 1023 exploit the flexibility of the transmit parameters and as seen 1024 in Fig. 13, they provide an SNR gain of about 5.1-7.3 dB over 1025 the conventional SM scheme at the BER of $10^{-5}$. Moreover, the 1026 TPC-aided SM achieves the best BER performance amongst all 1027 benchmark schemes, as seen in Fig. 13. This is mainly due to 1028 the fact that the PA-assisted SM and PRP-aided SM schemes 1029 are simplified versions of the TPC-aided SM scheme, which 1030 have a suboptimal BER performance. Moreover, the selection 1031 of TPC parameters is more flexible than that of ASM, because 1032 the modulation orders of ASM are selected from a discrete set, 1033 while the TPC parameters are chosen from the vast complex- 1034 valued field. The performance gain of the TPC-aided SM over 1035 ASM is explicitly seen in Fig. 13.

\section{Antenna Selection}

Antenna Selection (AS) constitutes another promising low- 1038 cost technique, since it enjoys the full-diversity benefits offered 1039 by MIMO architectures at the cost of requiring a low feedback 1040 rate. Due to its advantages, AS has been adopted in contempo- 1041 rary wireless systems such as IEEE 802.11n [136]. A detailed 1042 overview of AS techniques was presented in [136] and both 1043 the so-called norm-based selection and the successive selection 1044 scheme were detailed. Recently, a systematic overview of all 1045 physical and higher layer features of the LTE standard relying 1046 on Transmit AS (TAS) were presented in [137]. To be specific, 1047 TAS has been adopted by LTE for both its Frequency Division 1048 Duplexing (FDD) and Time Division Duplexing (TDD) modes 1049 of operation.

SM can also be beneficially combined with the AS technique 1051 for the sake of enhancing its performance. In recent years, 1052 several AS methods have been introduced and extended to the 1053 class of SM-MIMO systems with the goal of enhancing its 1054 capacity or its BER. For example, in [127], a TAS method based 1055 on exhaustive search was proposed for exploiting the available 1056 CSI. As natural extensions of the existing literature on TAS 1057 for spatial multiplexing systems, in [128], a low-complexity 1058 maximum-ED based TAS method and a maximum-capacity 1059 TAS method were investigated. Moreover, three closed-loop 1060 AS-aided SSK schemes were proposed in [126], which relied 1061 on the classic norm-based AS criterion, on the minimal PEP 1062 criterion and on their hybrid.

\section{Hybrid Adaptation and Other LA Schemes}

As mentioned in Section IV-A, ASM is capable of transmit- 1065 ting different number of bits over different TAs. Hence this 1066 scheme may achieve increased benefits due to the associated 1067 channel gain difference by exploiting it with the aid of dis- 1068 similar channel matrix column vectors [104]. For example, 1069 as shown in Algorithm 3, the number of bits carried by the 1070 conventional 4-QAM symbol is 2 in each SM symbol, while 1071 the number of bits conveyed by the TA indices is only one. 1072 The AM scheme is capable of varying this bit-mapping strategy 1073 according to the near-instantaneous channel conditions, while 1074 the TPC aided schemes [30], [103], [121]-[125] have to utilize 1075 a fixed modulation order and hence they may fail to achieve 1076 
1077 this level of flexibility. However, TPC exhibits an extra grade 1078 of flexibility, since it can have arbitrary coefficients.

1079 As discussed in the context of (4) and Fig. 9, apart from 1080 adapting the APM modes, LA-aided SM can also benefit from 1081 adapting the TPC parameters for the sake of improving the sys1082 tem's performance. For example, when a high power amplifier 1083 efficiency and a high transmission rate are required, the classic 1084 PSK scheme may be preferred to QAM in diverse SM-MIMO 1085 configurations both in terms of its BER and PAPR, because 1086 PSK may be conveniently combined with the above-mentioned 1087 PRP technique for creating a PRP-aided constant-modulus SM 1088 scheme. In this scheme, the APM constellation optimization 1089 technique of Section III may be efficiently combined with 1090 the TPC technique of Section IV-B for improving both the 1091 achievable energy efficiency and the BER performance.

\section{V. FURTHER SM-RELATED STUdiES}

\section{A. Cooperative SM-Related Systems}

1094 Cooperative techniques are capable of gleaning some of 1095 the advantages of classic multiple-antenna aided transmission 1096 techniques with the aid of cooperating single-antenna assisted 1097 nodes within a network [139]. Based on a philosophy similar to 1098 that of the STC-based schemes, relay-aided SM schemes have 1099 been proposed in [140]-[147]. For example, in [140], a decode1100 and-forward (DF) relaying aided coherent STSK system was 1101 proposed, where the dispersion-vector was activated based on 1102 cyclic redundancy checking (CRC)-assisted error detection. 1103 The proposed design is capable of adapting both the number of 1104 the RNs as well as the transmission rate and the achievable di1105 versity order, depending on the associated system requirements 1106 and channel conditions. Moreover, a differentially-encoded and 1107 non-coherently detected version of STSK was developed in 1108 [140], which dispenses with CSI estimation at all of the nodes, 1109 while retaining the benefits of the cooperative coherent STSK. 1110 In order to further improve the cooperative STSK's perfor1111 mance as well as to combat the effects of frequency-selective 1112 channels, in [141], Successive-Relaying (SR)aided cooperative 1113 multicarrier (MC) STSK was proposed. This technique invokes 1114 the selective DF and SR principles for the sake of recovering the 1115 half-duplex multiplexing loss while relying on the MC Code1116 Division Multiple Access (MC-CDMA) [148] principle for 1117 supporting multiple users, and simultaneously circumventing 1118 the dispersive effects of wireless channels. Moreover, in [142] 1119 a so-called Information Guided Transmission (IGT) scheme 1120 was employed for carrying out the random selection of the 1121 active nodes from the set of candidate Relay Nodes (RNs) for 1122 the sake of achieving a high relay throughput. Note that the 1123 above-mentioned SM-related cooperative systems may rely on 1124 single-antenna based transmissions at the Source-Node (SN), 1125 but some form of loose inter-relay synchronization (IRS) should 1126 be considered, unless the so-called Large-Area-Synchronized 1127 (LAS) spreading codes of [149], [150] are employed.

1128 Moreover, in [121], an Amplify-and-Forward (AF)-relaying1129 aided SSK scheme was conceived for reducing the number 1130 of TAs and for mitigating the effects of deep fading. More 1131 recently, Mesleh et al. [143], [144] invoked dual-hop AF and
DF relaying aided SSK schemes, which were characterized 1132 by the corresponding BER performance upper-bounds. How- 1133 ever, as mentioned in Section II, the throughput of the SSK- 1134 aided cooperative schemes may remain somewhat limited. To 1135 eliminate this impediment, a dual-hop cooperative SM scheme 1136 [145] was conceived for combining SSK with classic APM 1137 techniques for the sake of transmitting additional bits. More 1138 specifically, the spatial domain of dual-hop SM has been ex- 1139 ploited for transmitting additional information bits, hence this 1140 system may have the potential of providing substantial spectral 1141 efficiency and coding gains in the context of wireless relay 1142 networks. In [146], the SSK-MIMO principle is studied for 1143 the uplink of cellular networks. The source broadcasts its data 1144 packet to the available relays. The data packets are decoded by 1145 each relay individually and each decoded symbol is compared 1146 against unique identifiers of the relays. The specific relays 1147 that demodulate the data associated with their own identifier 1148 become active and transmit the associated SSK symbol to the 1149 destination. Hence, the set of relays act as a distributed spatial- 1150 constellation diagram for the source, similar to the SSK-MIMO 1151 communications concept with co-located TAs. The distributed 1152 encoding principle of [146] was then extended in [147] with 1153 the objective of improving the achievable bandwidth efficiency 1154 of half-duplex relaying. The associated transmission protocol 1155 is similar to that of [146], with one main exception, namely 1156 that active relays transmit the first data packet stored in their 1157 buffers during the second phase. This enables the relay to 1158 simultaneously transmit both the data received from the source 1159 and its own data. This is due to the fact that when a relay 1160 is active, the source data is conveyed by conventional APM 1161 modulation through this relay, while an additional data symbol 1162 can be implicitly mapped onto the relay's index. The results 1163 show that the adoption of a distributed SM-MIMO scheme is 1164 indeed capable of improving the attainable performance.

\section{B. SM-Related Systems for Frequency Selective Channels}

Despite its rich literature, the family of SM-related schemes 1167 has been predominantly investigated in the context of single- 1168 user flat fading channels. However, in high-rate SM-MIMO 1169 communication systems, the Inter-Symbol Interference (ISI) 1170 caused by multipath components of the frequency selective 1171 channel has to be considered.

Hence various SM-related systems have been investigated 1173 not only in the context of single carrier (SC) contexts [148] 1174 and but also in multi-carrier systems [151]. More specifically, 1175 in [55] the authors proposed the STFSK regime for overcoming 1176 the effects of dispersive channels, while striking a flexible 1177 trade-off between the attainable diversity and multiplexing 1178 gain. STFSK is capable of flexibly exploiting the available 1179 time-, space- and frequency-diversity, hence attaining an attrac- 1180 tive performance in frequency-selective fading environments. 1181 In [152], an OFDM-aided STSK system was proposed, which 1182 achieves almost the same BER performance as that of its single- 1183 carrier counterpart operating in a narrowband channel. More- 1184 over, in order to support high-rate multiuser transmissions, a 1185 novel multiuser STSK scheme was conceived for frequency- 1186 selective channels in [153], which was combined with the 1187 
1188 classic OFDMA/SC-FDMA techniques for the sake of con1189 verting the frequency-selective wideband channel to numerous 1190 parallel non-dispersive narrowband channels. In [154], an 1191 antenna-hopping space-division multiple-access aided SM 1192 scheme was advocated for exploiting the advantages of SM. 1193 For efficiently detecting this scheme, a range of linear and non1194 linear detection schemes have been investigated.

1195 Recently, SM-related schemes were investigated in a fre1196 quency selective channel by combining the classic Cyclic1197 Prefixed (CP) single carrier technique [155], [156], which is 1198 capable of avoiding the PAPR problem encountered in mul1199 ticarrier based systems. A comparison between CP-aided SC$1200 \mathrm{SM}$ and the CP-aided SM-OFDM systems was also presented 1201 for the sake of identifying the advantages of the single-carrier1202 SM scheme. Then, Rajashekar et al. further generalized the 1203 solutions of [156], where a Zero-Padded (ZP) single-carrier 1204 SM system was proposed for achieving the maximum attain1205 able multi-path diversity order with the aid of low-complexity 1206 single-stream ML detection. It was shown that the proposed ZP1207 aided SC-SM system provides beneficial system performance 1208 improvements compared to both the CP-aided SC-SM and the 1209 CP-aided SM-OFDM systems.

\section{C. The Energy-Efficient SM-Related Systems}

1211 Recently, the energy consumption issue in wireless commu1212 nication has attracted increasing attention, especially in MIMO1213 aided LTE and LTE-A networks [111], [157]. As a new kind of 1214 MIMO transmission technique and a promising candidate for 1215 future wireless applications and standards, SM can be realized 1216 by using a single RF front-end, hence it has a high power1217 efficiency [15]. However, how to further improve the energy1218 efficiency of SM-MIMO schemes is important in practical 1219 deployments.

1220 Some of the above-mentioned issues have been already 1221 investigated in [16], [17], [69], and [158]. More specifi1222 cally, in [16] the authors evaluated the energy efficiency of a 1223 multi-antenna assisted base station employing SM based on a 1224 realistic power consumption model. It was found that the SM1225 aided base station has a considerable power consumption gain 1226 compared to multi-RF chain aided MIMO arrangements. This 1227 advantage of SM was further confirmed in [17] by considering 1228 different base station types. Then, in [158], the energy con1229 sumption of a class of adaptive SM was evaluated. Moreover, 1230 in [69], an energy-efficient SM-MIMO scheme was designed, 1231 which relied on the Hamming coding and Huffman coding 1232 techniques. This scheme was capable of striking a flexible 1233 spectral-efficiency versus energy-efficiency tradeoff. Note that 1234 although the above-mentioned research demonstrated that SM 1235 constitutes an energy-efficient design [111], [157], the current 1236 research results are still preliminary and hence further investi1237 gations are required.

\section{CONCLUSION}

\section{A. Summary of the Paper}

1240 In this tutorial, we reviewed a range of recent research 1241 achievements on SM and its potential applications. We consid- ered some of its transceiver design aspects, the spatial constel- 1242 lation optimization, the associated link adaptation techniques, 1243 the distributed/cooperative system design issues and their ben- 1244 eficial combinations.

In Section II, we provided a rudimentary system overview 1246 of the conventional SM technique and its variants, empha- 1247 sizing the associated transceiver design techniques for strik- 1248 ing an attractive trade-off amongst the range of potentially 1249 conflicting system requirements. More specifically, the bit-to- 1250 symbol mapping principle of the SM transmitter was presented 1251 Section II-A. Then, various generalized versions of SM were 1252 introduced in Section II-B. Section II-C summarized the class 1253 of hard- and soft-detection techniques designed for SM-related 1254 schemes, which was roughly divided into four fundamental 1255 categories. In Section II-D, both the channel capacity and error 1256 performance metrics of SM-related schemes were summarized, 1257 which were used as a reference for the sake of highlighting 1258 the advantages of SM compared to other MIMO arrangements. 1259 These metrics were also used for system optimization by ex- 1260 ploiting the knowledge of CSI.

In Section III, the effects of APM schemes on the perfor- 1262 mance of SM were characterized and we proposed a class of 1263 star-QAM constellations for minimizing the system's BER. In 1264 Section IV, we introduced the family of limited-feedback aided 1265 LA techniques designed for SM-related schemes. Depending on 1266 the specific degree of freedom exploited, these techniques were 1267 divided into four types constituted by AM, TPC, AS and their 1268 hybrid techniques. Specifically, the near-instantaneously ASM 1269 scheme of Section IV-A has been proposed in [104], [115], 1270 and [119] for improving the attainable system performance, 1271 while maintaining a fixed average transmit rate with the aid 1272 of AM techniques. Moreover, the diagonal TPC scheme of 1273 Section IV-B has been proposed in [118] and [122] based 1274 on maximizing the FD for the family of SM-MIMO systems, 1275 where the transmitted symbols are appropriately pre-weighted 1276 according to the channel condition. Finally, we discussed a 1277 variety of other SM-related classes including those designed for 1278 frequency selective channels, for cooperative SM scenarios and 1279 for energy-efficient applications.

\section{B. Future Research Ideas}

In this paper, we considered only the minimum-distance 1282 based approach of extracting the LA parameters, in order to 1283 achieve beneficial performance improvements in the high-SNR 1284 regime. As further work, one can formulate and solve the LA 1285 problems by considering a range of other optimization criteria 1286 depending on the amount of channel state information available 1287 as well as on other system requirements, such as capacity- and 1288 SNR-optimized design rules [39]. Moreover, the integration of 1289 trellis coding as well as space-time block coding and other 1290 coding techniques [4] into the proposed LA schemes may also 1291 be further researched. Perhaps the most challenging of all is 1292 the design of non-coherent detection aided or blind-detection 1293 assisted schemes, which are capable of dispensing with channel 1294 information. These are particularly important in the context of 1295 relay-aided systems, where the source-relay channel cannot be 1296 readily estimated. 
1298

\section{ABEP}

1300 AM

1301 APM

1302 AS

1303 ASM

1304 BER

1305 BP-IGCH

$1306 \mathrm{CS}$

1307 CSI

1308 C-SM

1309 CSTSK

1310 DSTC

$1311 \mathrm{ED}$

$1312 \mathrm{EMF}$

1313 FD

1314 FSK

1315 FBE

1316 GSM

1317 G-STSK

1318 IAI

1319 IAS

$1320 \mathrm{IGCH}$

1321 ISI

1322 LA

1323 LTE

1324 MA-SM

1325 MAP

$1326 \mathrm{MF}$

1327 MIMO

1328 MISO

$1329 \mathrm{ML}$

1330 MMD

1331 MOS

1332 MRC

$1333 \mathrm{NMF}$

1334 OFDM

1335 OFDMA

1336 OFDM-IM

$1337 \mathrm{OH}-\mathrm{SM}$

1338 OSD

1339 OSDM

1340 PA

1341 PAPR

1342 PEP

1343 PRP

1344 PSK

1345 QAM

$1346 \mathrm{RF}$

1347 R-SM

1348 SC-FDMA

1349

$1350 \mathrm{SD}$

1351 SDM

1352 SFSK

1353 SIM

1354 SISO
VII. GLOSSARY

Average Bit Error Probability.

Adaptive Modulation.

Amplitude and Phase Modulation.

Antenna Selection.

Adaptive SM.

Bit Error Ratio.

Bit-Padding IGCH.

Compressed Sensing.

Channel State Information.

Concatenated SM.

Coherent STSK.

Differentially-encoded STC.

Euclidean distance.

Exhaustive-search MF.

Free Distance.

Frequency-Shift Keying.

Fractional Bit Encoded.

Generalized SM.

Generalized STSK.

Inter Antenna Interference.

Inter-Antenna-Synchronization.

Information-Guided Channel Hopping.

Inter-Symbol Interference.

Link adaptation.

Long-Term Evolution.

Multiple Active-SM.

Maximum a posteriori.

Matched Filter.

Multiple-Input Multiple-Output.

Multiple-Input Single-Output.

Maximum Likelihood.

Maximum-Minimum Distance.

Modulation Order Selection.

Maximum Ratio Combining.

Near-optimal MF.

Orthogonal Frequency-Division Multiplexing.

Orthogonal Frequency Division Multiple Access.

OFDM with Index Modulation.

Optimal Hybrid-SM.

Ordered SD.

Orthogonal Spatial-Division Multiplexing.

Power Allocation.

Peak-to-Average-Power Ratio.

Pairwise Error Probability.

Phase Rotation Precoding.

Phase Shift Keying.

Quadrature Amplitude Modulation.

Radio Frequency.

Receiver-SM.

Single-Carrier Frequency Division Multiple Access.

Sphere Decoding.

Spatial Division Multiplexing.

Space-Frequency Shift Keying.

Subcarrier-Index Modulation.

Single-Input Single-Output.
SM Spatial Modulation.

1355

STBC Space Time Block Codes. 1356

STC Space-Time Coding. 1357

STFSK Space-Time-Frequency Shift Keying. 1358

SSK $\quad$ Space Shift Keying. 1359

STSK Space-Time Shift Keying. $\quad 1360$

VBD $\quad$ Vector Based Detection. 1361

TA Transmit Antenna. 1362

TAS Transmit Antenna Selection. 1363

TC Trellis Coding. 1364

TCM Trellis Coded Modulation. 1365

TOSD-SM Time-Orthogonal Signal Design assisted SM. 1366

TPC Transmit Precoding. 1367

TMS Transmit Mode Switching. $\quad 1368$

USTM Unitary Space-Time Modulation. 1369

V-BLAST Vertical-Bell Laboratories Layered Space-Time. 1370
1371

[1] G. J. Foschini and M. J. Gans, "On limits of wireless communications 1372 in a fading environment when using multiple antennas," Wireless Pers. 1373 Commun., vol. 6, no. 3, pp. 311-335, Mar. 1998.

[2] I. E. Telatar, "Capacity of multi-antenna Gaussian channels," Eur. Trans. 1375 Telecommun., vol. 10, no. 6, pp. 585-595, Nov./Dec. 1999.

[3] V. Tarokh, N. Seshadri, and A. R. Calderbank, "Space-time codes for 1377 high data rate wireless communication: Performance criterion and code 1378 construction," IEEE Trans. Inf. Theory, vol. 44, no. 2, pp. 744-765, 1379 Mar. 1998.

1380

[4] L. Hanzo, O. Alamri, M. El-Hajjar, and N. Wu, Near-Capacity Multi- 1381 Functional MIMO Systems: Sphere-Packing, Iterative Detection, and 1382 Cooperation. Hoboken, NJ, USA: Wiley, 2009. 1383

[5] Q. Li et al., "MIMO techniques in WiMAX and LTE: A feature 1384 overview," IEEE Commun. Mag., vol. 48, no. 5, pp. 86-92, May 2010. 1385

[6] F. Boccardi et al., "Multiple-antenna techniques in LTE-advanced," 1386 IEEE Commun. Mag., vol. 50, no. 3, pp. 114-121, Mar. 2012.

[7] S. Sugiura, S. Chen, and L. Hanzo, "MIMO-aided near-capacity turbo 1388 transceivers: Taxonomy and performance versus complexity," IEEE 1389 Commun. Surveys Tuts., vol. 14, no. 2, pp. 421-442, Second Quarter 1390 2012.

1391

[8] S. Sugiura, S. Chen, and L. Hanzo, "A universal space-time architec- 1392 ture for multiple-antenna aided systems," IEEE Commun. Surveys Tuts., 1393 vol. 14, no. 2, pp. 401-420, Second Quarter 2012.

[9] J. Mietzner, R. Schober, L. Lampe, W. H. Gerstacker, and P. A. Hoeher, 1395 "Multiple-antenna techniques for wireless communications-A compre- 1396 hensive literature survey," IEEE Commun. Surveys Tuts., vol. 11, no. 2, 1397 pp. 87-105, Second Quarter 2009.

1398

[10] L. Zheng and D. N. C. Tse, "Diversity and multiplexing: A fundamental 1399 tradeoff in multiple-antenna channels," IEEE Trans. Inf. Theory, vol. 49, 1400 no. 5, pp. 1073-1096, May 2003.

1401

[11] R. Y. Mesleh, H. Haas, S. Sinanovic, C. W. Ahn, and S. Yun, "Spatial 1402 modulation," IEEE Trans. Veh. Technol., vol. 57, no. 4, pp. 2228-2241, 1403 Jul. 2008.

1404

[12] Y. Yang and B. Jiao, "Information-guided channel-hopping for high 1405 data rate wireless communication," IEEE Commun. Lett., vol. 12, no. 4, 1406 pp. 225-227, Apr. 2008.

1407

[13] L. Hanzo, S. X. Ng, T. Keller, and W. Webb, Quadrature Amplitude 1408 Modulation: From Basics to Adaptive Trellis-Coded, Turbo-Equalised 1409 and Space-Time Coded OFDM, CDMA and MC-CDMA Systems. 1410 Hoboken, NJ, USA: Wiley, 2004.

[14] M. Di Renzo, H. Haas, and P. M. Grant, "Spatial modulation for 1412 multiple-antenna wireless systems: A survey," IEEE Commun. Mag., 1413 vol. 49, no. 12, pp. 182-191, Dec. 2011.

[15] M. Di Renzo, H. Haas, A. Ghrayeb, S. Sugiura, and L. Hanzo, "Spatial 1415 modulation for generalized MIMO: Challenges, opportunities and im- 1416 plementation," Proc. IEEE, vol. 102, no. 1, pp. 56-103, Jan. 2014.

[16] A. Stavridis, S. Sinanovic, M. Di Renzo, H. Haas, and P. M. Grant, "An 1418 energy saving base station employing spatial modulation," in Proc. IEEE 1419 Int. Workshop Comput.-Aided Model. Anal. Des. Commun. Links Netw., 1420 Barcelona, Spain, Sep. 2012, pp. 231-235.

[17] A. Stavridis, S. Sinanovic, M. Di Renzo, and H. Haas, "Energy 1422 evaluation of spatial modulation at a multi-antenna base station," in 1423 
Proc. IEEE Veh. Technol. Conf.-Fall, Barcelona, Spain, Sep. 2013, pp. 1-5.

[18] T. L. Marzetta, "Noncooperative cellular wireless with unlimited numbers of base station antennas," IEEE Trans. Wireless Commun., vol. 9, no. 11, pp. 3590-3600, Nov. 2010.

[19] F. Rusek et al., "Scaling up MIMO: Opportunities and challenges with very large arrays," IEEE Signal Proces. Mag., vol. 30, no. 1, pp. 40-46, Jan. 2013.

[20] R. Mesleh, H. Elgala, and H. Haas, "Optical spatial modulation," IEEE/OSA J. Optical Commun. Netw., vol. 3, no. 3, pp. 234-244, Mar. 2011.

[21] W. Popoola, E. Poves, and H. Haas, "Error performance of generalised space shift keying for indoor visible light communications," IEEE Trans. Commun., vol. 61, no. 5, pp. 1968-1976, May 2013.

[22] W. Popoola, E. Poves, and H. Haas, "Spatial pulse position modulation for optical communications," EEE/OSA J. Lightw. Technol., vol. 30, no. 18, pp. 2948-2954, Sep. 2012.

23] C. Vladeanu, R. Lucaciu, and A. Mihaescu, "Optical spatial modulation for indoor wireless communications in presence of inter-symbol interference," in Proc. IEEE Int. Symp. Electron. Telecommun., Nov. 2012, pp. 183-186.

24] E. Poves, W. Popoola, H. Haas, J. Thompson, and D. Cardenas, "Experimental results on the performance of optical spatial modulation systems," in Proc. IEEE Veh. Technol. Conf._Fall, Quebec City, QC, Canada, Sep. 2012, pp. 1-5.

25] A. Younis et al., "Performance of spatial modulation using measured real-world channels," in IEEE Veh. Technol. Conf.-Fall, Las Vegas, NV, USA, 2013, pp. 1-5.

26] N. Serafimovski et al., "Practical implementation of spatial modulation," IEEE Trans. Veh. Technol., vol. 62, no. 9, pp. 4511-4523, Nov. 2013.

27] M. Di Renzo and H. Haas, "A general framework for performance analysis of space shift keying (SSK) modulation for MISO correlated Nakagami-m fading channels," IEEE Trans. Commun., vol. 58, no. 9, pp. 2590-2603, Sep. 2010

28] M. Di Renzo and H. Haas, "Space shift keying (SSK-) MIMO over correlated Rician fading channels: Performance analysis and a new method for transmit-diversity," IEEE Trans. Commun., vol. 59, no. 1, pp. 116129, Jan. 2011.

29] M. Di Renzo and H. Haas, "Bit error probability of SM-MIMO over generalized fading channels," IEEE Trans. Veh. Technol., vol. 61, no. 3, pp. 1124-1144, Mar. 2012

[30] M. Di Renzo and H. Haas, "Improving the performance of space shift keying (SSK) modulation via opportunistic power allocation," IEEE Commun. Lett., vol. 14, no. 6, pp. 500-502, Jun. 2010.

31] M. Di Renzo and H. Haas, "On transmit-diversity for spatial modulation MIMO: Impact of spatial-constellation diagram and shaping filters at the transmitter," IEEE Trans. Veh. Technol., vol. 62, no. 6, pp. 2507-2531, Jul. 2013.

[32] M. Di Renzo and H. Haas, "Bit error probability of space shift keying MIMO over multiple-access independent fading channels," IEEE Trans. Veh. Technol., vol. 60, no. 8, pp. 3694-3711, Oct. 2011.

[33] M. Di Renzo and H. Haas, "Space shift keying (SSK) modulation with partial channel state information: Optimal detector and performance analysis over fading channels," IEEE Trans. Commun., vol. 58, no. 11, pp. 3196-3210, Nov. 2010.

34] M. Di Renzo, D. De Leonardis, F. Graziosi, and H. Haas, "Space shift keying (SSK-) MIMO with practical channel estimates," IEEE Trans. Commun., vol. 60, no. 4, pp. 998-1012, Apr. 2012.

35] S. M. Alamouti, "A simple transmit diversity technique for wireless communications," IEEE J. Sel. Areas Commun., vol. 16, no. 8, pp. 14511458, Oct. 1998.

[36] P. W. Wolniansky, G. J. Foschini, G. D. Golden, and R. A. Valenzuela, "V-BLAST: An architecture for realizing very high data rates over the rich-scattering wireless channel," in Proc. ISSSE, Pisa, Italy, 1998, pp. $295-300$.

37] B. Hassibi and B. M. Hochwald, "High-rate codes that are linear in space and time," IEEE Trans. Inf. Theory, vol. 48, no. 7, pp. 1804-1824, Jul. 2002.

[38] L. Hanzo, J. Blogh, and S. Ni, 3G, HSPA and FDD Versus TDD Networking: Smart Antennas and Adaptive Modulation. Hoboken, NJ, USA: Wiley, 2008.

39] D. J. Love et al., "An overview of limited feedback in wireless communication systems," IEEE J. Sel. Areas Commun., vol. 26, no. 8, pp. 13411365, Oct. 2008.

[40] C. B. Chae, A. Forenza, R. W. Heath, Jr., M. R. Mckay, and I. B. Collings, "Adaptive MIMO transmission techniques for broadband wireless communication systems," IEEE Commun. Mag., vol. 48, no. 5, 1501 pp. 112-118, May 2010.

[41] S. Catreux, V. Erceg, D. Gesbert, and R. W. Heath, Jr., "Adaptive mod- 1503 ulation and MIMO coding for broadband wireless data networks," IEEE 1504 Commun. Mag., vol. 40, no. 6, pp. 108-115, Jun. 2002.

[42] H. Haas, E. Costa, and E. Schultz, "Increasing spectral efficiency by 1506 data multiplexing using antennas arrays," in Proc. IEEE Int. Symp. Pers., 1507 Indoor, Mobile Radio Commun., Sep. 2002, vol. 2, pp. 610-613. 1508

[43] S. Sugiura, S. Chen, and L. Hanzo, "Coherent and differential 1509 space-time shift keying: A dispersion matrix approach," IEEE Trans. 1510 Commun., vol. 58, no. 11, pp. 3219-3230, Nov. 2010.

[44] J. Jeganathan, A. Ghrayeb, L. Szczecinski, and A. Ceron, "Space 1512 shift keying modulation for MIMO channels," IEEE Trans. Wireless 1513 Commun., vol. 8, no. 7, pp. 3692-3703, Jul. 2009.

[45] Y. Chau and S.-H. Yu, "Space modulation on wireless fading chan- 1515 nels," in Proc. IEEE Veh. Technol. Conf._Fall, Atlantic City, NJ, USA, 1516 Oct. 2001, vol. 3, pp. 1668-1671.

[46] J. Jeganathan, A. Ghrayeb, and L. Szczecinski, "Generalized space shift 1518 keying modulation for MIMO channels," in Proc. IEEE Int. Symp. Pers., 1519 Indoor, Mobile Radio Commun., Cannes, France, Sep. 2008, pp. 1-5. 1520

[47] J. Fu, C. Hou, W. Xiang, L. Yan, and Y. Hou, "Generalised spatial modu- 1521 lation with multiple active transmit antennas," in Proc. IEEE Globecom 1522 Workshops, Miami, FL, USA, Dec. 2010, pp. 839-844.

[48] A. Younis, N. Serafimovski, R. Mesleh, and H. Haas, "Generalised 1524 spatial modulation," in Proc. Signals, Syst. Comput., Pacific Grove, CA, 1525 USA, Nov. 2010, pp. 1498-1502.

[49] J. Wang, S. Jia, and J. Song, "Generalised spatial modulation system 1527 with multiple active transmit antennas and low complexity detection 1528 scheme," IEEE Trans. Wireless Commun., vol. 11, no. 4, pp. 1605-1615, 1529 Apr. 2012.

[50] E. Başar, Ü. Aygölü, E. Panayırc1, and H. V. Poor, "Space-time block 1531 coded spatial modulation," IEEE Trans. Commun., vol. 59, no. 3, 1532 pp. 823-832, Mar. 2011.

[51] R. Y. Mesleh, M. Di Renzo, H. Haas, and P. M. Grant, "Trellis coded spa- 1534 tial modulation," IEEE Trans. Wireless Commun., vol. 9, no. 7, pp. 2349- 1535 2361, Jul. 2010.

[52] E. Başar, Ü. Aygölü, E. Panayırc1, and H. V. Poor, "New trellis code 1537 design for spatial modulation," IEEE Trans. Wireless Commun., vol. 10, 1538 no. 8, pp. 2670-2680, Aug. 2011.

[53] E. Başar, Ü. Aygölü, E. Panayırcı, and H. V. Poor, "Super-orthogonal 1540 trellis-coded spatial modulation," IET Commun., vol. 6, no. 17, 1541 pp. 2922-2932, Nov. 2012.

[54] S. Sugiura, S. Chen, and L. Hanzo, "Generalized space-time shift keying 1543 designed for flexible diversity-, multiplexing- and complexity-tradeoffs," 1544 IEEE Trans. Wireless Commun., vol. 10, no. 4, pp. 1144-1153, 1545 Apr. 2011.

[55] H. A. Ngo, C. Xu, S. Sugiura, and L. Hanzo, "Space-time-frequency 1547 shift keying for dispersive channels," IEEE Signal Process. Lett., vol. 18, 1548 no. 3, pp. 177-180, Mar. 2011.

1549

[56] H. A. Ngo and L. Hanzo, "Area spectral efficiency of soft-decision 1550 space-time-frequency shift-keying-aided slow-frequency-hopping mul- 1551 tiple access," IEEE Trans. Veh. Technol., vol. 61, no. 3, pp. 1433-1439, 1552 Mar. 2012.

[57] L. Hanzo, M. Münster, B. J. Choi, and T. Keller, OFDM and MC-CDMA 1554 for Broadband Multi-User Communications, WLANs and Broadcasting. 1555 Hoboken, NJ, USA: Wiley, 2003. 1556

[58] R. Abu-alhiga and H. Haas, "Subcarrier-index modulation OFDM," in 1557 Proc. IEEE Int. Sym. Pers., Indoor Mobile Radio Commun., Tokyo, 1558 Japan, Sep. 2009, pp. 177-181.

[59] E. Başar, Ü. Aygölü, E. Panayırc1, and H. V. Poor, "Orthogonal frequency 1560 division multiplexing with index modulation," in Proc. IEEE Global 1561 Commun. Conf., Anaheim, CA, USA, Dec. 2012, pp. 4741-4746. 1562

[60] R. A. Scholtz, "Multiple access with time-hopping impulse modulation," 1563 in Proc. Mil. Commun. Conf., Boston, MA, USA, Oct. 1993, vol. 2, 1564 pp. $447-450$.

[61] A. Latif and N. D.Gohar, "On the PAPR reduction of hybrid QAM/FSK 1566 (HQFM) OFDM transceiver,” J. Appl. Sci., vol. 8, no. 6, pp. 1061-1066, 1567 2008.

[62] S. Song et al., "A channel hopping technique I: Theoretical studies 1569 on band efficiency and capacity," in Proc. IEEE Int. Conf. Commun., 1570 Circuits Syst., Chengdu, SiChuan, Jun. 2004, vol. 1, pp. 229-233. 1571

[63] R. Y. Mesleh, H. Haas, C. W. Ahn, and S. Yun, "Spatial modulation-A 1572 new low complexity spectral efficiency enhancing technique," in IEEE 1573 Int. Conf. Commun. Netw. China, Beijing, China, Oct. 2006, pp. 1-5. 1574

[64] N. Serafimovski, M. Di Renzo, S. Sinanović, R. Y. Mesleh, and H. Haas, 1575 "Fractional bit encoded spatial modulation (FBE-SM)," IEEE Commun. 1576 Lett., vol. 14, no. 5, pp. 429-431, May 2010. 
[65] M. Di Renzo and H. Haas, "Performance comparison of different spatial modulation schemes in correlated fading channels," in Proc. IEEE Int. Conf. Commun., Cape Town, South Africa, May 2010, pp. 1-6.

[66] Y. Yang and S. Aissa, "Bit-padding information guided channel hopping," IEEE Commun. Lett., vol. 15, no. 2, pp. 163-165, Feb. 2011.

67] Q. Qu, A. Wang, Z. Nie, and J. Zheng, "Block mapping spatial modulation scheme for MIMO systems," J. China Univ. Posts Telecommun., vol. 18, no. 5, pp. 30-36, Oct. 2011.

[68] P. Zhang, D. Yuan, and H. Zhang, "A novel spatial modulation scheme over correlated fading channels," J. Commun., vol. 7, no. 11, pp. 847857, Nov. 2012.

[69] R. Y. Chang, S.-J. Lin, and W.-H. Chung, "Energy efficient transmission over space shift keying modulated MIMO channels," IEEE Trans. Commun., vol. 60, no. 10, pp. 2950-2959, Oct. 2012.

[70] R. Y. Chang, S.-J. Lin, and W.-H. Chung, "New space shift keying modulation with Hamming code-aided constellation design," IEEE Wireless Commun. Lett., vol. 1, no. 1, pp. 2-5, Feb. 2012.

[71] P.-H. Kuo, "SCRAD modulation: High data rate space shift keying scheme permitting reduced-complexity detector," IET Electron. Lett., vol. 48, no. 24, pp. 1567-1569, Nov. 2012.

[72] J. Jeganathan, A. Ghrayeb, and L. Szczecinski, "Spatial modulation: Optimal detection and performance analysis," IEEE Commun. Lett., vol. 12, no. 8, pp. 545-547, Aug. 2008.

[73] S. U. Hwang, S. Jeon, S. Lee, and J. Seo, "Soft-output ML detector for spatial modulation OFDM systems," IEICE Electron. Exp., vol. 6, no. 19, pp. 1426-1431, Sep. 2009.

[74] S. Chen, S. Sugiura, and L. Hanzo, "Semi-blind joint channel estimation and data detection for space-time shift keying systems," IEEE Signal Process. Lett., vol. 17, no. 12, pp. 993-996, Dec. 2010.

[75] M. X. Guo, C. Jia, and Y. H. Shen, "Detection algorithm for spatial modulation system under unconstrained channel," in Proc. IEEE Int. Conf. Commun. Technol., Nanjing, China, Nov. 2010, pp. 458-461.

[76] A. Younis, R. Y. Mesleh, H. Haas, and P. Grant, "Reduced complexity sphere decoder for spatial modulation detection receivers," in Proc. IEEE Global Commun. Conf., Miami, FL, USA, Dec. 2010, pp. $1-5$.

[77] A. Younis, M. Di Renzo, R. Y. Mesleh, and H. Haas, "Sphere decoding for spatial modulation," in Proc. IEEE Int. Conf. Commun., Kyoto, Japan, Jun. 2011, pp. 1-6.

78] P. Yang, Y. Xiao, Q. Tang, B. Zhou, and S. Q. Li, "A low complexity ordered sphere decoding algorithm for spatial modulation," IEICE Trans. Commun., vol. E95-B, no. 07, pp. 2494-2497, Jul. 2012.

[79] A. Younis, S. Sinanovic, M. Di Renzo, R. Y. Mesleh, and H. Haas, "Generalised sphere decoding for spatial modulation," IEEE Trans. Commun, vol. 61, no. 7, pp. 2805-2815, Jul. 2013.

[80] S. Sugiura, C. Xu, S. X. Ng, and L. Hanzo, "Reduced-complexity coherent versus non-coherent QAM-aided space-time shift keying," IEEE Trans. Commun., vol. 59, no. 11, pp. 3090-3101, Nov. 2011.

[81] C. Xu, S. Sugiura, S. X. Ng, and L. Hanzo, "Reduced-complexity softdecision aided space-time shift keying," IEEE Signal Process. Lett., vol. 18 , no. 10, pp. 547-550, Oct. 2011

[82] C. Vladeanu, A. Martian, A. F. Paun, and E. El Assad, "A new ML detector for trellis-coded spatial modulation using hard and soft estimates," in Proc. IEEE Int. Symp. Electron. Telecommun., Timisoara, Romania, Nov. 2012, pp. 143-146.

83] P. Yang, Y. Xiao, L. Li, Q. Tang, and S. Li, "An improved matched-filter based detection algorithm for space-time shift keying systems," IEEE Signal Process. Lett., vol. 19, no. 5, pp. 271-274, May 2012.

[84] H. Xu, "Simple low-complexity detection schemes for $M$-ary quadrature amplitude modulation spatial modulation," IET Commun., vol. 6, no. 17, pp. 2840-2847, Nov. 2012.

[85] N. R. Naidoo, H. Xu, and T. Quazi, "Spatial modulation: Optimal detector asymptotic performance and multiple-stage detection," IET Commun., vol. 5, no. 10, pp. 1368-1376, Jul. 2011.

[86] S. Sugiura, C. Xu, S. X. Ng, and L. Hanzo, "Reduced-complexity iterative-detection aided generalized space-time shift keying," IEEE Trans. Veh. Technol., vol. 61, no. 8, pp. 3656-3664, Oct. 2012.

[87] Q. Tang, Y. Xiao, P. Yang, Q. Yu, and S. Li, "A new low-complexity near-ML detection algorithm for spatial modulation," IEEE Wireless Commun. Lett., vol. 2, no. 1, pp. 90-93, Feb. 2013.

88] C. Xu, S. Sugiura, S. X. Ng, and L. Hanzo, "Spatial modulation and space-time shift keying: Optimal performance at a reduced detection complexity," IEEE Trans. Commun., vol. 61, no. 1, pp. 206-216, Jan. 2013.

[89] J. Wang, S. Jia, and J. Song, "Signal vector based detection scheme for spatial modulation," IEEE Commun. Lett., vol. 16, no. 1, pp. 19-21, Jan. 2012.
[90] J. Zheng, "Signal vector based list detection for spatial modulation," 1655 IEEE Wireless Commun. Lett., vol. 1, no. 4, pp. 265-267, Aug. 2013.

[91] C.-M. Yu et al., "Compressed sensing detector design for space shift key- 1657 ing in MIMO systems," IEEE Commun. Lett., vol. 16, no. 10, pp. 1556- 1658 1559, Oct. 2012.

[92] M. Kadir, S. Sugiura, J. Zhang, S. Cheng, and L. Hanzo, "OFDMA/ 1660 SC-FDMA-aided space-time shift keying for dispersive multiuser sce- 1661 narios," IEEE Trans. Veh. Technol., vol. 62, no. 1, pp. 408-414, 1662 Jan. 2013.

1663

[93] E. Viterbo and J. Boutros, "A universal lattice code decoder for fading 1664 channels," IEEE Trans. Inf. Theory, vol. 45, no. 5, pp. 1639-1642, 1665 Jul. 1999 .

[94] L. Hanzo, T. H. Liew, and B. L. Yeap, Turbo Coding, Turbo Equalisa- 1667 tion, and Space-Time Coding for Transmission Over Fading Channels. 1668 Hoboken, NJ, USA: Wiley, 2002.

[95] B. M. Hochwald and T. L. Marzetta, "Unitary space-time modulation for 1670 multiple-antenna communications in Rayleigh flat fading," IEEE Trans. 1671 Inf. Theory, vol. 46, no. 2, pp. 543-564, Mar. 2000.

1672

[96] B. L. Hughes, "Differential space-time modulation," IEEE Trans. Inf. 1673 Theory, vol. 46, no. 7, pp. 2567-2578, Nov. 2000.

1674

[97] C. Xu, S. Sugiura, S. X. Ng, and L. Hanzo, "Reduced-complexity non- 1675 coherently detected differential space-time shift keying," IEEE Signal 1676 Process. Lett., vol. 18, no. 3, pp. 153-156, Mar. 2011.

1677

[98] L. Xiao, Y. Xiao, P. Yang, and S. Li, "Low-complexity non-coherent 1678 detection for spatial modulation OFDM systems," in Proc. IEEE Int. 1679 Conf. Commun. Technol., Chengdu, China, 2012, pp. 469-473. 1680

[99] R. Zhang and L. Hanzo, "A unified treatment of superposition coding 1681 aided communications: Theory and practice," IEEE Commun. Surveys 1682 Tuts., vol. 13, no. 3, pp. 503-520, Third Quarter 2011.

1683

[100] S. X. Ng and L. Hanzo, "On the MIMO channel capacity of multidimen- 1684 sional signal sets," IEEE Trans. Veh. Technol., vol. 55, no. 2, pp. 528- 1685 536, Mar. 2006.

1686

[101] X. Guan, Y. Xiao, Y. Cai, and W. Yang, "On the mutual information and 1687 precoding for spatial modulation with finite alphabet," IEEE Wireless 1688 Commun. Lett., vol. 2, no. 4, pp. 383-386, Aug. 2013.

[102] J. G. Proakis, Digital Communications, 4th ed. New York, NY, USA: 1690 McGraw-Hill, 2004.

[103] T. Handte, A. Muller, and J. Speidel, "BER analysis and optimization of 1692 generalized spatial modulation in correlated fading channels," in Proc. 1693 IEEE Veh. Technol. Conf., Anchorage, AK, USA, Sep. 2009, pp. 1-5. 1694

[104] P. Yang, Y. Xiao, Y. Yi, and S. Li, "Adaptive spatial modulation for 1695 wireless MIMO transmission systems," IEEE Commun. Lett., vol. 15, 1696 no. 6, pp. 602-604, Jun. 2011. 1697

[105] A. Goldsmith, Wireless Communications. Cambridge, U.K.: 1698 Cambridge Univ. Press, 2005.

[106] M. S. Alaouni and M. K. Simon, Digital Communications Over Fading 1700 Channels, 2nd ed. Hoboken, NJ, USA: Wiley, 2005.

[107] E. Başar, Ü. Aygölü, E. Panayırcı, and H. V. Poor, "Performance of 1702 spatial modulation in the presence of channel estimation errors," IEEE 1703 Commun. Lett., vol. 16, no. 2, pp. 176-179, Feb. 2012.

[108] S. S. Ikki and R. Mesleh, "A general framework for performance analysis 1705 of space shift keying (SSK) modulation in the presence of Gaussian 1706 imperfect estimations," IEEE Commun. Lett., vol. 16, no. 2, pp. 228- 1707 230, Feb. 2012

[109] S. Sugiura and L. Hanzo, "Effects of channel estimation on spatial 1709 modulation," IEEE Signal Process. Lett., vol. 19, no. 12, pp. 805-808, 1710 Dec. 2012.

[110] P. Yang, Y. Xiao, L. Dan, and S. Li, "An improved gradient-based PAPR 1712 reduction method for space shift keying (SSK)-OFDM systems," IEICE 1713 Trans. Commun., vol. E94-B, no. 12, pp. 3532-3539, Dec. 2011.

[111] Z. Hasan, H. Boostanimehr, and V. K. Bhargava, "Green cellular net- 1715 works: A survey, some research issues and challenges," IEEE Commun. 1716 Surveys Tuts., vol. 13, no. 4, pp. 524-540, Nov. 2011.

[112] S. Sugiura and L. Hanzo, "On the joint optimization of dispersion ma- 1718 trices and constellations for near-capacity irregular precoded space-time 1719 shift keying," IEEE Trans. Wireless Commun., vol. 12, no. 1, pp. 380- 1720 387, Jan. 2013.

[113] J. M. Luna-Rivera, D. U. Campos-Delgado, and M. G. Gonzalez-Perez, 1722 "Constellation design for spatial modulation," Procedia Technol., vol. 7, 1723 pp. 71-78, 2013.

[114] M. Maleki, H. R. Bahrami, S. Beygi, M. Kafashan, and N. H. Tran, 1725 "Space modulation with CSI: Constellation design and performance 1726 evaluation," IEEE Trans. Veh. Technol., vol. 62, no. 4, pp. 1623-1634, 1727 May 2013.

[115] P. Yang et al., "Link adaptation for spatial modulation with limited 1729 feedback," IEEE Trans. Veh. Technol., vol. 61, no. 8, pp. 3808-3813, 1730 Oct. 2012 
1732 [116] K. Ishibashi, W. Shin, H. Ochiai, and V. Tarokh, "A peak power efficient 1733 cooperative diversity using star-QAM with coherent/noncoherent detec1734 tion," IEEE Trans. Wireless Commun., vol. 12, no. 5, pp. 2137-2147, 1735 May 2013

1736 [117] L. Lampe, "Noncoherent coded modulation," Ph.D. dissertation, Dept. 1737 Elect. Eng., University of Erlangen, Erlangen, Germany, 2002.

1738 [118] P. Yang et al., "Star-QAM signaling constellations for spatial modula1739 tion," IEEE Trans. Veh. Technol.

1740 [119] P. Yang et al., "Simplified adaptive spatial modulation for limited1741 feedback MIMO,” IEEE Trans. Veh. Technol., vol. 62, no. 6, pp. 26561742 2666, Jul. 2013.

1743 [120] B. M. Mthethwa and H. Xu, "Adaptive $M$-ary quadrature amplitude 1744 spatial modulation," IET Commun., vol. 6, no. 18, pp. 3098-3108, 1745 Dec. 2012.

1746 [121] D. Yang, C. Xu, L.-L. Yang, and L. Hanzo, "Transmit-diversity-assisted 1747 1748

1764 [126] W.-H. Chung and C.-Y. Hung, "Multi-antenna selection using space

1765

1766

1767

1768

1769

1770

1771

1772

1773

1774

1775

1776

1777

1778

1779

1780

1781

1782

1783 [

1784

1785

1786

1787

1788

1789

1790 [134] J. Kim, B. W. Kim, and D. J. Park, "Mitigation of transmit correlation 1791 for TIMO spatial multiplexing through phase rotation," IEEE Commun. 1792 Lett., vol. 12, no. 2, pp. 118-120, Feb. 2008.

1793 [135] A. E. Ekpenyong and Y. Huang, "Feedback constraints for adaptive 1794 transmission," IEEE Signal Process. Mag., vol. 24, no. 3, pp. 69-78, 1795 May 2007

1796 [136] S. Sanayei and A. Nosratinia, "Antenna selection in MIMO systems," 1797 IEEE Commun. Mag., vol. 42, no. 10, pp. 68-73, Oct. 2004.

1798 [137] N. B. Mehta, A. F. Molisch, and S. Kashyap, "Antenna selection in LTE: 1799 From motivation to specification," IEEE Commun. Mag., vol. 50, no. 10, 1800 pp. 144-150, Oct. 2012.

1801 [138] R. W. Heath, Jr., S. Sandhu, and A. Paulraj, "Switching between diversity 1802 and multiplexing in MIMO systems," IEEE Trans. Commun., vol. 53, 1803 no. 6, pp. 962-968, Jun. 2005.

1804 [139] J. N. Laneman, D. N. C. Tse, and G. W. Wornell, "Cooperative diversity 1805 in wireless networks: Efficient protocols and outage behavior," IEEE 1806 Trans. Inf. Theory, vol. 50, no. 12, pp. 3062-3080, Dec. 2004.

1807 [140] S. Sugiura, S. Chen, H. Haas, P. M. Grant, and L. Hanzo, "Coher1808 ent versus non-coherent decode-and-forward relaying aided coopera- tive space-time shift keying," IEEE Trans. Commun., vol. 59, no. 6, 1809 pp. 1707-1719, Jun. 2011.

1810

[141] P. Zhang, S. Chen, and L. Hanzo, "Differential space-time shift keying 1811 aided successive relaying assisted cooperative multiuser CDMA," IEEE 1812 Trans. Veh. Technol., vol. 62, no. 5, pp. 2156-2169, Jun. 2013.

1813

[142] Y. Yang and S. Aissa, "Information-guided transmission in decode- 1814 and forward relaying systems: Spatial exploitation and throughput en- 1815 hancement," IEEE Wireless Commun., vol. 10, no. 7, pp. 2341-2351, 1816 Jul. 2011.

1817

[143] R. Mesleh, S. S. Ikki, E. M. Aggoune, and A. Mansour, "Performance 1818 analysis of space shift keying (SSK) modulation with multiple coopera- 1819 tive relays," EURASIP J. Adv. Signal Process., vol. 2014, no. 1, pp. 1-10, 1820 Sep. 2012.

1821

[144] R. Mesleh, S. S. Ikki, and M. Alwakeel, "Performance analysis of space 1822 shift keying with amplify and forward relaying," IEEE Commun. Lett., 1823 vol. 15, no. 12, pp. 1350-1352, Dec. 2011.

1824

[145] N. Serafimovski, S. Sinanović, M. Di Renzo, and H. Haas, "Dual-hop 1825 spatial modulation (Dh-SM)," in Proc. VTC -Spring, Budapest, Hun- 1826 gary, May 15-18, 2011, pp. 1-5.

[146] S. Narayanan, M. Di Renzo, F. Graziosi, and H. Haas, "Distributed space 1828 shift keying for the uplink of relay-aided cellular networks," in Proc. 1829 IEEE Int. Workshop Comput.-Aided Model. Anal. Des. Commun. Links 1830 Netw., Barcelona, Spain, Sept. 2012, pp. 17-19.

1831

[147] S. Narayanan, M. Di Renzo, F. Graziosi, and H. Haas, "Distributed 1832 spatial modulation for relay networks," in Proc. IEEE Veh. Technol. 1833 Conf._Fall, Las Vegas, USA, Sep. 2013, pp. 130-134. 1834

[148] L. Hanzo, L.-L. Yang, E.-L. Kuan, and K. Yen, Single and Multicarrier 1835 DS-CDMA: Multi-User Detection, Space-Time Spreading, Synchronisa- 1836 tion, Networking, and Standards. Hoboken, NJ, USA: Wiley, 2003. 1837

[149] S. Sugiura, S. X. Ng, L. Kong, S. Chen, and L. Hanzo, "Quasi- 1838 synchronous cooperative networks," IEEE Veh. Technol. Mag., vol. 7, 1839 no. 4, pp. 66-76, Dec. 2012.

1840

[150] H. Wei, L.-L. Yang, and L. Hanzo, "Iterference-free broadband sin- 1841 gle and multicarrier DS-CDMA," IEEE Commun. Mag., vol. 4, no. 3, 1842 pp. 68-73, Feb. 2005.

1843

[151] L. Hanzo, J. Akhtman, L. Wang, and M. Jiang, MIMO-OFDM for 1844 LTE, WiFi and WiMAX: Coherent Versus Non-Coherent and Cooperative 1845 Turbo Transceivers. Hoboken, NJ, USA: Wiley, 2010.

1846

[152] M. I. Kadir, L. Li, S. Chen, and L. Hanzo, "Successive-relaying-aided 1847 decode-and-forward coherent versus noncoherent cooperative multicar- 1848 rier space-time shift keying," IEEE Trans. Veh. Technol., vol. 62, no. 6, 1849 pp. 2544-2557, Jul. 2013.

[153] M. Driusso, F. Babich, M. I. Kadir, and L. Hanzo, "OFDM aided 1851 space-time shift keying for dispersive downlink channels," in IEEE Veh. 1852 Technol. Conf._Fall, Quebec City, QC, QC, Sept. 2012, pp. 1-5. 1853

[154] L. L. Yang, "Signal detection in antenna-hopping space-division 1854 multiple-access systems with space-shift keying modulation," IEEE 1855 Trans. Sig. Process., vol. 60, no. 1, pp. 351-366, Jan. 2012.

[155] P. Som and A. Chockalingam, "Spatial modulation and space shift key- 1857 ing in single carrier communication," in Proc. IEEE PIMRC, Sydney, 1858 NSW, Australia, 2012, pp. 1991-1996.

1859

[156] R. Rajashekar, K. V. S. Hari, and L. Hanzo, "Spatial modulation aided 1860 zero-padded single carrier transmission for dispersive channels," IEEE 1861 Trans. Commun., vol. 61, no. 6, pp. 2318-2329, Jun. 2013.

1862

[157] D. Feng et al., "A survey of energy-efficient wireless communications," 1863 IEEE Commun. Surveys Tuts., vol. 15, no. 1, pp. 167-178, Nov. 2013. 1864

[158] X. Wu, S. Sinanovic, M. Di Renzo, and H. Haas, "Base station energy 1865 consumption for transmission optimised spatial modulation (TOSM) 1866 in correlated channels," in Proc. IEEE 17th Int. Workshop CAMAD, 1867 Barcelona, Spain, 2012, pp. 261-265.

1868

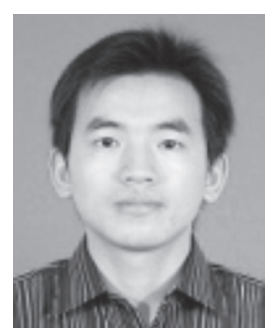

Ping Yang received the B.E., M.E., and Ph.D. de- 1869 grees from the University of Electronic Science and 1870 Technology of China, Chengdu, China, in 2006, 1871 2009 , and 2013, respectively. He is currently with 1872 the National Key Laboratory of Science and Tech- 1873 nology on Communications, University of Electronic 1874 Science and Technology of China. He has published 1875 AQ3 more than 20 international journals and international 1876 conference papers. His research interests include 1877 MIMO systems, space-time coding, and communi- 1878 cation signal processing. 


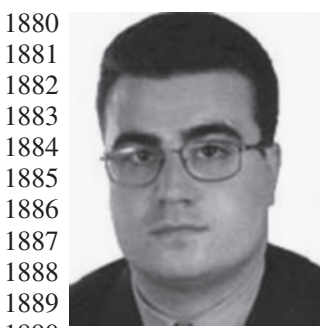

Marco Di Renzo (S'05-AM'07-M'09-SM'14) was born in L'Aquila, Italy, in 1978. He received the Laurea (cum laude) and Ph.D. degrees in electrical and information engineering from the University of L'Aquila, L'Aquila, in 2003 and 2007, respectively, and the Habilitation à Diriger des Recherches (HDR) from the University of Paris-Sud XI, Paris, France, in 2013.

Since January 2010, he has been a Tenured Academic Researcher ("Chargé de Recherche Titulaire") with the French National Center for Scientific Re1891 search (CNRS), as well as a faculty member of the Laboratory of Signals 1892 and Systems (L2S), a joint research laboratory of the CNRS, the École 1893 Supérieure d'Électricité (SUPÉLEC), and the University of Paris-Sud XI. His 1894 main research interests are in the area of wireless communications theory.

1895 Dr. Di Renzo currently serves an Editor of the IEEE COMMUNICATIONS 1896 LETTERS and of the IEEE TRANSACTIONS ON COMMUNICATIONS (Wireless 1897 Communications-Heterogeneous Networks Modeling and Analysis). He was 1898 the recipient of a special mention for the outstanding five-year (1997-2003) 1899 academic career, University of L'Aquila; the THALES Communications fel1900 lowship (2003-2006), University of L'Aquila; the 2004 Best Spin-Off Com1901 pany Award, Abruzzo Region, Italy; the 2006 DEWS Travel Grant Award, 1902 University of L'Aquila; the 2008 Torres Quevedo Award, Ministry of Sci1903 ence and Innovation, Spain; the "Dérogation pour l'Encadrement de Thèse" 1904 (2010), University of Paris-Sud XI; the 2012 IEEE CAMAD Best Paper 1905 Award; the 2012 IEEE WIRELESS COMMUNICATIONS LETTERS Exemplary 1906 Reviewer Award; the 2013 IEEE VTC-Fall Best Student Paper Award; the 19072013 Network of Excellence NEWCOM\# Best Paper Award; the 2013 IEEE 1908 Transactions on Vehicular Technology Top Reviewer Award; the 19092013 IEEE COMSOC Best Young Researcher Award for Europe, Middle East, 1910 and Africa (EMEA Region); and the 2014 IEEE ICNC Single Best Paper Award 1911 Nomination (Wireless Communications Symposium).

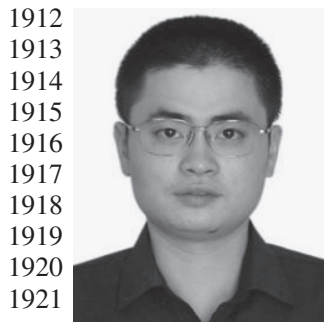

Yue Xiao received the Ph.D. degree in communication and information systems from the University of Electronic Science and Technology of China, Chengdu, China, in 2007. He is currently an Associate Professor with University of Electronic Science and Technology of China. He has published more than 30 international journals and been involved in several projects in Chinese Beyond 3G Communication R\&D Program. His research interests are in the area of wireless and mobile communications.

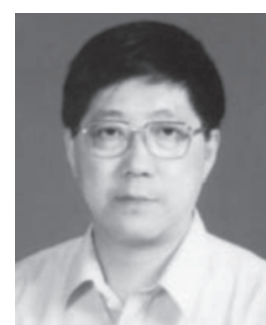

Shaoqian $\mathbf{L i}$ received the B.Eng. degree in electrical 1922 engineering from the American University of Beirut, 1923 Beirut, Lebanon, in 2004 and the M.Sc. degree 1924 in radio frequency communication systems and the 1925 $\mathrm{Ph} . \mathrm{D}$. degree in wireless communications from the 1926 University of Southampton, Southampton, U.K., in 1927 2005 and 2008, respectively. Following his Ph.D. 1928 degree, he joined Imagination Technologies as a Re- 1929 search Engineer, where he worked on designing and 1930 developing the BICM peripherals in Imagination's 1931 multi-standard communications platform, which re- 1932 sulted in several patent applications. In January 2012, he joined the School of 1933 Electronics and Computer Science, University of Southampton, as a Lecturer 1934 in the Communications, Signal Processing and Control Research Group. He 1935 has published a Wiley-IEEE book and in excess of 40 journal and international 1936 conference papers. His research interests include machine-to-machine commu- 1937 nications, millimeter-wave communications, large-scale MIMO, cooperative 1938 communications, and radio over fiber systems. Dr. Li was the recipient of 1939 several academic awards.

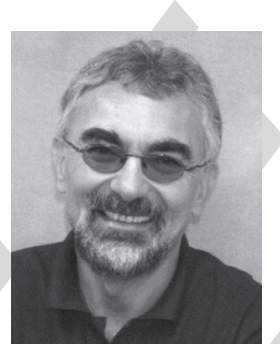

Lajos Hanzo received the M.S. degree in electronics 1941 and the Ph.D. degree from the Technical University 1942 of Budapest, Budapest, Hungary, in 1976 and 1983, 1943 respectively; the D.Sc. degree from the University of 1944 Southampton, Southampton, U.K., in 2004; and the 1945 "Doctor Honoris Causa" degree from the Technical 1946 University of Budapest in 2009.

During his 37-year career in telecommunications, 1948 he has held various research and academic posts in 1949 Hungary, Germany, and the U.K. Since 1986, he has 1950 been with the School of Electronics and Computer 1951 Science, University of Southampton, U.K., where he holds the Chair in 1952 Telecommunications. He is currently directing a 100-strong academic research 1953 team, working on a range of research projects in the field of wireless multimedia 1954 communications sponsored by industry, the Engineering and Physical Sciences 1955 Research Council (EPSRC) U.K., the European Research Council's Advanced 1956 Fellow Grant, and the Royal Society's Wolfson Research Merit Award. During 1957 2008-2012, he was a Chaired Professor with Tsinghua University, Beijing 1958 China. He is an enthusiastic supporter of industrial and academic liaison and 1959 offers a range of industrial courses. He has successfully supervised more than 1960 80 Ph.D. students, coauthored 20 John Wiley/IEEE Press books on mobile 1961 radio communications totaling in excess of 10000 pages, and published more 1962 than 1400 research entries at IEEE Xplore. He has more than 19000 citations. 1963 His research is funded by the European Research Council's Senior Research 1964 Fellow Grant. For further information on research in progress and associated 1965 publications, please refer to http://www-mobile.ecs.soton.ac.uk.

Dr. Hanzo is a Fellow of the Royal Academy of Engineering, the Institution 1967 of Engineering and Technology, and the European Association for Signal 1968 Processing. He is also a Governor of the IEEE Vehicular Technology Society. 1969 During 2008-2012, he was the Editor-in-Chief of the IEEE Press. He has served 1970 as the Technical Program Committee Chair and the General Chair of IEEE 1971 conferences, has presented keynote lectures, and has been awarded a number 1972 of distinctions. 


\section{AUTHOR QUERIES}

\section{AUTHOR PLEASE ANSWER ALL QUERIES}

AQ1 = Please provide publication update in Ref. [118].

AQ2 = Please provide page range in Ref. [124].

AQ3 = This was added as given in the author's affiliation information. Is this correct? If not, please provide his current affiliation data.

\section{END OF ALL QUERIES}

